\title{
Quantum filaments in dipolar Bose-Einstein condensates
}

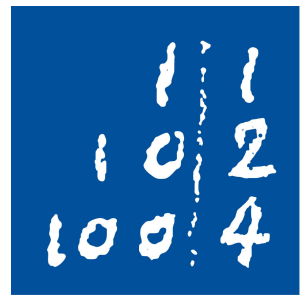
Leibniz
Universität
Hannover

Von der Fakultät für Mathematik und Physik

der Gottfried Wilhelm Leibniz Universität Hannover

\author{
zur Erlangung des Grades \\ Doktor der Naturwissenschaften \\ Dr. rer. nat.
}

genehmigte Dissertation von

Dipl. Phys. Falk Wächtler

geboren am 28.08.1985 in Dessau, Deutschland 
Tag der Promotion : 12.12 .2016 


\section{Abstract}

The field of dipolar Bose-Einstein condensates has attracted in recent years a large interest in the community of ultracold gases. Due to the anisotropic and long-ranged nature of the dipole-dipole interaction differ the properties of these gases in many aspects from purely contact interacting systems including the existence of rotonic excitations and the formation of stable quantum filaments. In this thesis we study dipolar Bose-Einstein condensates in the weakly interacting regime focusing on the existence of quantum droplets, their stability and collective modes.

We investigate the Bogoliubov excitation spectrum of a dipolar condensate close to instability for various trapping geometries and study the mode causing a dynamical instability in such a system in the mean-field regime. This reveals a crossover from condensates undergoing phononic to rotonic collapses with the most unstable mode becoming increasingly local. Simulations of the first stages of the post-destabilization dynamics open a way to probe these properties directly in experiments by monitoring the condensate shape.

Furthermore we show that the dipole-driven collapse of Bose-Einstein condensates caused by long-wavelength excitations can be arrested by quantum fluctuations of smaller wavelengths. We discuss the conditions in which the perturbative quantum fluctuation term becomes crucial for the static and dynamic properties in these systems demonstrating a general effect in strongly dipolar gases which will become important in future experiments. We compare our theoretical results directly with experiments of quantum droplets in dysprosium and erbium, showing the close connection of the dynamics of these systems, and the role of three-body losses in the formation of droplet crystals in dysprosium or short lived macro droplets in erbium.

Finally, we investigate the properties of a single quantum droplet. We study the different ground state solutions and map their existence in phase space identifying a region of multistability. The evolution from one state to the other in this regime reveals a phase transition of first order which can only be found in pancake-shaped traps. We show that the quantum filaments are self-bound for large particle numbers or small scattering length values, map their stability boundary, and study their destabilization mechanism via their collective oscillations. We also investigate the lowest-lying excitations of trapped droplets in general and show an increasing decoupling of the modes deeper in the droplet regime.

Keywords: ultracold quantum gases, quantum fluctuations, dipolar interactions 


\section{Kurzzusammenfassung}

Das Forschungsgebiet der dipolaren Bose-Einstein Kondensate hat in den letzten Jahren großes Interesse im Feld der ultrakalten Gase auf sich gezogen. Aufgrund der anisotropen und langreichweitigen Natur der Dipol-Dipol-Wechselwirkung unterscheiden sich die Eigenschaften dieser Gase in vielen Aspekten von denen rein Kontaktwechselwirkender. Dies beinhaltet die Existenz von rotonischen Anregungen und die Bildung stabiler Quantenfilamente. In dieser Doktorarbeit untersuchen wir Bose-Einstein Kondensate im schwach wechselwirkenden Regime und fokussieren uns dabei auf die Existenz von Quantenfilamenten, deren Stabilität und ihren kollektiven Anregungen.

Wir analysieren für verschiedene Fallengeometrien das Bogoliubovspektrum eines dipolaren Kondensates nahe der Instabilitätsgrenze und untersuchen im Molekularfeldregime die Anregung, die die dynamische Instabilität in diesem System verursacht. Dies enthüllt einen kontinuierlichen Übergang von phononisch zu rotonisch getriebenen Kollapsen, wobei die instabilste Anregung einen zunehmend lokalen Charakter erhält. Simulationen der ersten Stufen der Dynamik nach der Destabilisierung ermöglichen es diese Eigenschaften direkt durch das Dichteprofil in Experimenten zu untersuchen.

Des weiteren zeigen wir, dass der durch die Dipol-Dipol-Wechselwirkung getriebene Kollaps langwelliger Anregungen eines Bose-Einstein Kondensates von Quantenfluktuationen kürzerer Wellenlänge gestoppt werden kann. Wir diskutieren die Bedingungen in welchen der störungstheoretische Quantenfluktuationsterm entscheidend für die statischen und dynamischen Eigenschaften dieser Systeme wird und demonstrieren damit einen allgemeinen Effekt stark dipolarer Gase. Dies wird in zukünftigen Experimenten wichtig sein. Wir vergleichen unsere theoretischen Ergebnisse direkt mit Experimenten von Quantenfilamenten in Dysprosium und Erbium. Dabei zeigen wir die enge Verknüpfung der Dynamik dieser Systeme und der Rolle von Dreikörperverlusten in der Bildung von Kristallen von Quantenfilamenten in Dysprosium oder kurzlebigen Makrofilamenten in Erbium.

Schließlich untersuchen wir die Eigenschaften eines einzelnen Quantenfilamentes. Wir analysieren die unterschiedlichen Grundzustandslösungen, erstellen ein Phasenraumdiagramm und machen ein Gebiet der Multistabilität ausfindig. Die Entwicklung von einem Zustand zum anderen in diesem Regime offenbart einen Phasenübergang erster Ordnung, der nur in Fallen mit einer abgeflachten Geometrie gefunden werden kann. Wir zeigen, dass die Quantenfilamente selbstgebunden für große Teilchenzahlen oder kleine Streulängen sind, kartographieren ihre Stabilitätsgrenze und untersuchen durch die kollektiven Anregungen ihren Instabilitätsmechanismus. Des weiteren untersuchen wir allgemein die energetisch niegrigstliegende Anregung eines selbstgebunden Quantenfilamente und zeigen außerdem eine zunehmende Entkopplung der radialen und axialen kollektiven Anregungen im Bereich der Quantenfilamente. 
Schlagwörter: ultrakalte Quantengase, Quantenfluktuationen, dipolare Wechselwirkungen 


\section{Contents}

1. Bose-Einstein condensation 9

1.1. The Ideal Bose Gas . . . . . . . . . . . . . . . . . . . . . 10

1.2. Weak Interactions and Scattering . . . . . . . . . . . . 11

1.3. Mean-field description . . . . . . . . . . . . . . . . . . 12

1.4. Thomas-Fermi regime . . . . . . . . . . . . . . . . . . . . . . . . . . . . . . 15

1.5. Stability and Collapse . . . . . . . . . . . . . . 16

1.6. Excitations . . . . . . . . . . . . . . . . 17

2. Dipolar Bose-Einstein Condensates 19

2.1. The dipole-dipole interaction . . . . . . . . . . . . . . . . 20

2.2. Geometrical Stability . . . . . . . . . . . . . . . . . 22

2.3. Mean-field description and ground state properties . . . . . . . . . 23

2.4. Collapse dynamics in Chromium . . . . . . . . . . . . . 24

2.5. Excitations . . . . . . . . . . . . . . . . 25

2.6. Roton Excitations . . . . . . . . . . . . . . . . 26

3. Phonon-to-roton crossover 31

3.1. Stability diagram . . . . . . . . . . . . . . . 31

3.2. Bogoliubov spectrum . . . . . . . . . . . . . . . . 33

3.2.1. Framework . . . . . . . . . . . . . 33

3.2.2. Phonon-to-roton crossover . . . . . . . . . . . 35

3.3. Post-destabilization dynamics . . . . . . . . . . . . . 36

3.4. Conclusions . . . . . . . . . . . . . . . . . . . . . . . . . . 39

4. Higher order corrections 41

4.1. Lee-Huang-Yang corrections . . . . . . . . . . . . . . . . 41

4.2. Lee-Huang-Yang corrections in binary Bose mixtures . . . . . . . 43

4.3. LHY corrections in uniform dipolar BECs . . . . . . . . . . . . . . 4 45

4.4. LHY correction in non-uniform dipolar BECs . . . . . . . . . . . 45

5. Quantum filaments 49

5.1. Framework . . . . . . . . . . . . . . . . . . . . 49

5.2. Quench Experiments . . . . . . . . . . . . 50

5.3. Properties of a single droplet . . . . . . . . . . . . . . . . . 51

5.4. Statistics of the droplet cloud . . . . . . . . . . . . . 54

5.5. Conclusion . . . . . . . . . . . . . . . . 56

6. Ground-state properties and elementary excitations of quantum $\begin{array}{ll}\text { filaments } & 57\end{array}$

6.1. Framework . . . . . . . . . . . . . . . . 57

6.2. Ground-state properties . . . . . . . . . . . . . 59

6.2.1. Droplet versus mean-field solution . . . . . . . . . . . . 59 
6.2.2. Multistability . . . . . . . . . . . . . . 60

6.2.3. Crossover from the mean-field to the droplet solution . . . 61

6.3. Self-bound condensates . . . . . . . . . . . . . . . 62

6.4. Excitations . . . . . . . . . . . . . . . 6 65

6.5. Conclusions . . . . . . . . . . . . . . . 67

7. Quantum fluctuations in an erbium BEC 69

7.1. Framework . . . . . . . . . . . . . . . . . 69

7.2. Loss dynamics . . . . . . . . . . . . . . . . . . . 71

7.3. Collective axial excitation . . . . . . . . . . . . . . 74

7.4. Expansion dynamics . . . . . . . . . . . . . . . . . 76

7.5. Conclusion . . . . . . . . . . . . . . . . . . 79

8. Conclusions and Outlook 81

A. Validity of the local density approximation 85

$\begin{array}{ll}\text { B. Numerical procedure to calculate the Bogoliubov spectrum } & 87\end{array}$

B.1. Matrix Elements for $f_{+} \ldots \ldots$. . . . . . . . . . . . . 87

B.2. Calculating the Matrix Elements for $\mathrm{f}_{-}$. . . . . . . . . . . 89

B.3. Calculating the effective momentum . . . . . . . . . . . . . . . 90

$\begin{array}{ll}\text { Bibliography } & 92\end{array}$

$\begin{array}{ll}\text { Curriculum Vitae } & 105\end{array}$

$\begin{array}{ll}\text { Publications } & 107\end{array}$

$\begin{array}{ll}\text { Acknowledgements } & 109\end{array}$ 


\section{Bose-Einstein condensation}

In 1924 Satyendra Nath Bose deduced Planck's law for the first time in a clean way by incorporating that identical quantum particles are actually indistinguishable, in stark contrast to classical objects which have distinct trajectories. Einstein immediately realized its importance, translated Bose's paper and published it in "Zeitschrift für Physik" [1]. Afterwards he applied this idea to massive particles and postulated that if the temperature of a bosonic gas is reduced below a critical value a phase transition occurs [2]. The particles form an aggregate of individually indistinguishable coherent particles, which is nowadays known as Bose-Einstein condensate (BEC).

Unfortunately, the experimental realization of a BEC proved to be a challenging task. Either large densities or small temperatures are needed to enter the quantum regime and large densities also imply strong interparticle interactions. Thereby a significant amount of particles occupies higher energy states and the number of particles in the BEC, even when it exists, is small. For instance, the condensate fraction in liquid helium is only around 10\% [3]. The successful idea to solve this problem is to reduce the density of the gas significantly which on the other hand also reduces the necessary temperatures into the $\mu \mathrm{K}$ regime. Thus, the invention of novel cooling techniques was required, which took several decades [4-6]. The first BEC's were created in 1995 in the experimental groups of Eric Cornell and Carl Wieman with rubidium [7] as well as in Wolfgang Ketterle's group with sodium [8]. For this work Cornell, Wieman and Ketterle were awarded in 2001 with the Nobel prize in physics.

The physics of Bose-Einstein condensates has attracted a lot of interest in the physics community. It enables to investigate the condensate wave function by means of optical methods making an abstract concept of quantum physics visible to the human eye. Additionally, quantum effects can be studied in a very controllable way and described quite well within a mean-field approach. Although BEC's are dilute, interactions play an important role as the temperatures are very low and can be tuned by relatively simple means. Moreover, there exists a large number of methods to manipulate the condensate by means of lasers and magnetic fields. As real collisional processes are negligible, interference phenomena can also be studied.

In this chapter we discuss some key concepts on Bose-Einstein condensation relevant for the rest of this Thesis, focusing on the ideal Bose gas in section 1.1. Section 1.2 is dedicated to the description of the interactions followed by a discussion of the mean-field treatment in section 1.3 and the Thomas-Fermi regime in section 1.4. Section 1.5 is dedicated to the stability properties and section 1.6 to the elementary excitations. 


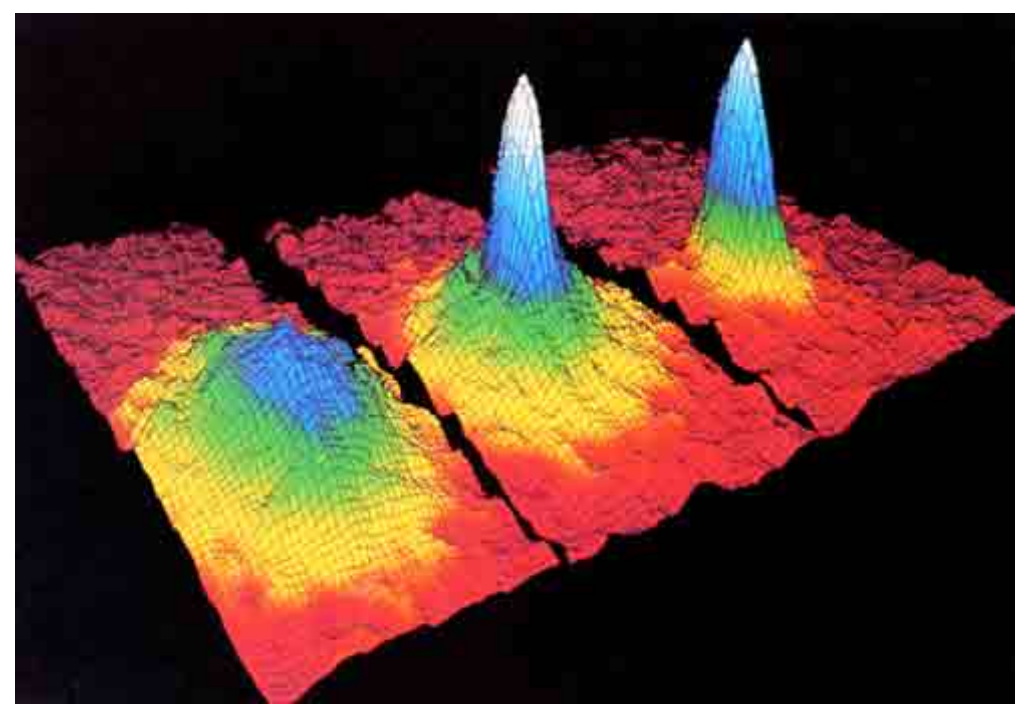

Figure 1.1.: Observation of Bose-Einstein condensation by showing the velocity distribution of a gas of rubidium atoms. The temperature in the left figure is above the critical value for Bose-Einstein condensation. Hence only a thermal Gaussian distribution can be seen. In the figure in the center is the temperature lowered below the critical value revealing a condensate peak. In the right figure are almost all atoms condensed so that a pure BEC was produced. The picture was reprinted from Ref. [9].

\subsection{The Ideal Bose Gas}

A Bose gas is typically studied in the grand canonical ensemble with the chemical potential $\mu$ and the absolute temperature $T$ as the thermodynamic variables. The occupation number of a single particle quantum state with energy $E_{k}$ can be derived using the grand canonical partition function and the symmetry of bosonic many-body quantum states yielding [10]

$$
N_{k}=\frac{1}{e^{\beta\left(E_{k}-\mu\right)}-1},
$$

where $\beta=1 /\left(k_{B} T\right)$ and $k_{B}$ is the Boltzmann constant. However, in a typical experiment is not the chemical potential given, but rather the total number of particles. Therefore, $\mu$ has to be extracted from $N=\sum_{k} N_{k}$. As $N_{k}$ has to be a positive quantity the chemical potential is necessarily smaller than the energy of any state $k$, which is an expression that bosons seemingly feel an attractive potential between them. Therefore bosons tend to occupy states which are already occupied. The sum in Eq. (1.1) can be calculated by replacing it by an integral which is valid if the thermal energy $k_{B} T$ is much larger than the energy spacing between the single particle energy levels. However, with such a procedure the ground state is not described accurately [10]. As it plays a crucial role at low temperatures we separate its contribution from the sum leading to [10]

$$
N=N_{0}+\frac{V}{\lambda_{T}^{3}} g_{3 / 2}(z)
$$


where $N_{0}$ is the number of particles in the ground state, $V$ is the volume of the gas and $\lambda_{T}=\sqrt{\frac{2 \pi \hbar^{2}}{M k_{B} T}}$ is the thermal de Broglie wavelength with $M$ the mass of the particles. The function $g_{3 / 2}(z)=\frac{2}{\sqrt{\pi}} \int_{0}^{\infty} d x \frac{\sqrt{x}}{z^{-1} e^{x}-1}$ is a mathematical standard integral containing the fugacity $z=e^{\beta \mu}$.

Equation (1.2) implies that if the temperature and the density of the gas $(n=$ $N / V)$ are sufficient to ensure that $\lambda_{T}^{3} n>g_{3 / 2}(z)$, i.e. large density or small temperature, a finite fraction of the particles occupies the ground state of the system and the gas is Bose-Einstein condensed (Fig. (1.1)). This condition can be physically interpreted as that the condensation begins when the average distance between the particles becomes comparable to their thermal de Broglie wavelength $\lambda_{T}$. The density in typical experiments ranges from $10^{19} \mathrm{~m}^{-3}$ to $10^{21} \mathrm{~m}^{-3}$ and the temperature from $50 \mathrm{nK}$ to $1 \mu \mathrm{K}$. It is important to stress at this point that a BEC is not simply a Bose gas with a high fraction of the ground state particles, but a new form of matter separated by a phase transition from a thermal gas.

However the details of Bose-Einstein condensation are much more subtle than what one would naively expect. It took nearly 40 years from the first discussion of Bose-Einstein condensation made by Einstein to realize that phase coherence and broken gauge symmetry [11] are the essence of a BEC. Moreover, in an interacting system it is not clear what is meant by a macroscopic occupation of the ground state due to the fact that the eigenstate of a many-body Hamiltonian are in general not products of single particle states. Therefore in 1956 Penrose and Onsager introduced a definition for Bose-Einstein condensation based on off-diagonal long-range order holding for both interacting and non-interacting systems [12]: A gas is Bose-Einstein condensed if the correlation function $G\left(\mathbf{r}, \mathbf{r}^{\prime}\right)=\int d \mathbf{r}_{2} \ldots d \mathbf{r}_{N} \Psi_{0}\left(\mathbf{r}, \mathbf{r}_{2}, \ldots, \mathbf{r}_{N}\right) \Psi_{0}\left(\mathbf{r}^{\prime}, \mathbf{r}_{2}, \ldots, \mathbf{r}_{N}\right)$ remains finite if $\left|\mathbf{r}-\mathbf{r}^{\prime}\right| \rightarrow \infty$, where $\Psi_{0}\left(\mathbf{r}_{1}, \mathbf{r}_{2}, \ldots, \mathbf{r}_{N}\right)$ is the ground state of the manybody Hamiltonian $H_{N}$ describing the Bose gas. This definition characterizes the off-diagonal long-range order in a BEC implying phase coherence over the whole condensate. The meaning and importance of this definition became gradually clear in the following years through the works of Goldstone [13], Yang [14], and Anderson [15].

\subsection{Weak Interactions and Scattering}

In order to describe interactions let us first have a look at the basics of scattering theory. Consider two atoms interacting via the general potential $V_{\text {int }}\left(\mathbf{r}_{1}-\mathbf{r}_{2}\right)$, whose range is finite and the subscripts 1,2 denote the individual atoms. The Hamiltonian in the center of mass frame takes the form

$$
\hat{H}=\frac{p^{2}}{2 M_{r}}+V_{\text {int }}(\mathbf{r})
$$

where $M_{r}=M_{1} M_{2} /\left(M_{1}+M_{2}\right)$ is the reduced mass. In a sufficiently dilute gas is the mean interatomic distance much larger than the typical range of the interatomic potential $r_{0}$, i.e. $n^{-1 / 3} \ll r_{0}$. Therefore the wave functions of the particles at mean interparticle separation are not influenced by the interactions 


\section{Bose-Einstein condensation}

and the Schrödinger equation can be solved in this limit with the result [16]

$$
\Psi(\mathbf{r})=e^{\mathrm{i} \mathbf{r} \cdot \mathbf{k}}+f_{k}(\vartheta, \varphi) \frac{e^{\mathrm{i} \mathbf{k} \cdot \mathbf{r}}}{r},
$$

where $f_{k}(\vartheta, \varphi)=-\frac{M}{2 \pi \hbar^{2}} \int d^{3} r^{\prime} e^{-\mathrm{i} k \frac{\mathbf{r} \cdot \mathbf{r}^{\prime}}{r}} V_{\text {int }}\left(\mathbf{r}^{\prime}\right) \Psi\left(\mathbf{r}^{\prime}\right)$ is called the scattering amplitude. Eq. (1.4) describes the incoming wave and a scattered spherical wave with an angle dependent factor $f_{k}$. Note that if $n r_{0}^{3} \ll 1$ is satisfied the shape of the potential is irrelevant and the asymptotic form of the wave function in Eq. (1.4) is sufficient to characterize the atomic interactions. The scattering amplitude $f_{k}$ characterizes the whole collisional process and can be calculated in the limit of low temperatures, i.e. when the thermal de Broglie wavelength $\lambda_{T}$ is much larger than $r_{0}$, by means of a partial wave expansion with the result [17]

$$
f_{k \rightarrow 0}=-a_{\mathrm{s}} .
$$

This means that the scattered wavefunction is spherically symmetric irrespective of the underlying potential as the details of the short-ranged interactions do not matter in the above mentioned regime. The whole scattering process is therefore characterized by a single parameter, the scattering length $a_{\mathrm{s}}$. With this knowledge the complicated interatomic interaction potentials can be replaced by the much simpler contact pseudopotential

$$
V_{\text {int }}\left(\mathbf{r}-\mathbf{r}^{\prime}\right)=g \delta^{(3)}\left(\mathbf{r}-\mathbf{r}^{\prime}\right),
$$

where $g=\frac{4 \pi \hbar^{2} a_{\mathrm{s}}}{M}[18]$ and $\delta^{(3)}(\mathbf{r})$ is the three-dimensional delta function. The treatment of the pseudopotential was first developed by Enrico Fermi in 1936 [19].

Important to note is that the scattering length can be tuned by magnetic or optic Feshbach resonances [20-24], enabling to control the contact interaction strength in an experiment. A Feshbach resonance happens if a bound state (closed channel) is energetically close to the energy of two scattering atoms. The presence of the bound state changes the wave functions during the scattering event leading to a shift of the scattering length. In a magnetic Feshbach resonance the potentials of the open and closed channels are shifted to resonance by changing the magnetic field $B$ as their energy depends on the Zeeman effect. In the case of an optical Feshbach resonance the closed and open channels are coupled directly via laser light. The scattering length can be tuned to arbitrary values and is given by [17]

$$
a_{\mathrm{s}}(B)=a_{\mathrm{bg}}\left(1-\frac{\Delta}{B-B_{0}}\right),
$$

where $a_{\mathrm{bg}}$ is the background scattering length which summarizes the effect of the direct scattering process in the open channel. $B_{0}$ is the resonance position at which the scattering length diverges and $\Delta$ is the width of the Feshbach resonance.

\subsection{Mean-field description}

The many-body Hamiltonian for $\mathrm{N}$ interacting bosonic particles takes the form

$$
\begin{aligned}
\hat{H}= & \int d^{3} r \hat{\Psi}^{\dagger}(\mathbf{r})\left(-\frac{\hbar^{2} \nabla^{2}}{2 M}+V_{\mathrm{tr}}(\mathbf{r})\right) \hat{\Psi}(\mathbf{r}) \\
& +\frac{1}{2} \int d^{3} r d^{3} r^{\prime} \hat{\Psi}^{\dagger}(\mathbf{r}) \hat{\Psi}^{\dagger}\left(\mathbf{r}^{\prime}\right) V_{\text {int }}\left(\mathbf{r}-\mathbf{r}^{\prime}\right) \hat{\Psi}\left(\mathbf{r}^{\prime}\right) \hat{\Psi}(\mathbf{r}),
\end{aligned}
$$




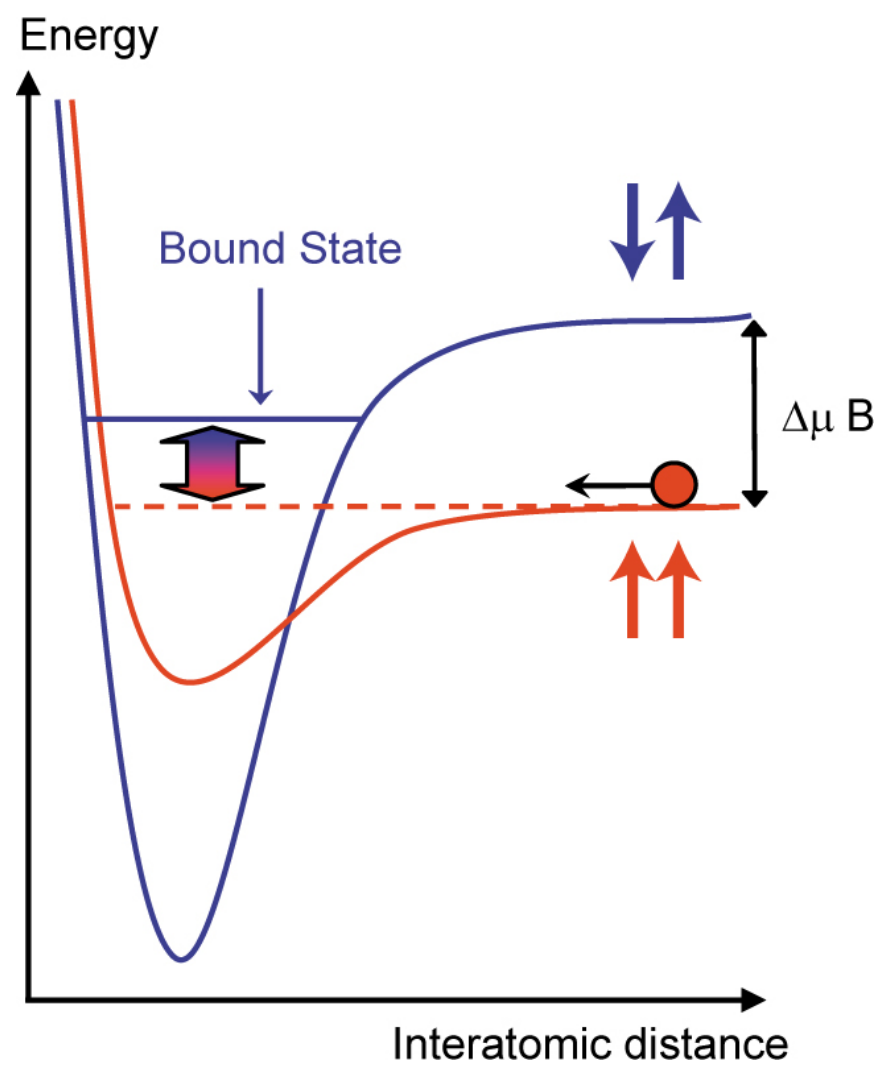

Figure 1.2.: Schematic representation of a Feshbach resonance. The red curve represents the scattering potential and the blue curve the interaction potential of the bound state. A resonance occurs when the energy of a bound states equals the initial energy of the scattered atoms. The picture was reprinted from Ref. [25].

where $\hat{\Psi}$ is the field operator and $\hat{\Psi}^{\dagger}$ its adjoint satisfying the usual bosonic commutation relations $\left[\hat{\Psi}(\mathbf{r}), \hat{\Psi}\left(\mathbf{r}^{\prime}\right)\right]=0,\left[\hat{\Psi}^{\dagger}(\mathbf{r}), \hat{\Psi}^{\dagger}\left(\mathbf{r}^{\prime}\right)\right]=0$, and $\left[\hat{\Psi}(\mathbf{r}), \hat{\Psi}^{\dagger}\left(\mathbf{r}^{\prime}\right)\right]=$ $\delta^{(3)}\left(\mathbf{r}-\mathbf{r}^{\prime}\right)$. The first term in Eq. (1.8) describes the kinetic and trapping contribution with $V_{\text {tr }}$ being the external trap potential. The second term represents the interactions described by the two-body potential $V_{\text {int }}$ for which we employ the contact pseudopotential Eq. (1.6). Three-body and higher interactions can be neglected as the density in typical BEC experiments is small. However, in some cases it is necessary to consider three-body interactions as for example when the condensate collapses or particle losses occur in very long lasting experiments. We will come to this issue in the following sections.

Although the dynamics and characteristics of such a system are fully determined by the Hamiltonian (1.8) it is very hard to get analytical or numerical results. Even powerful Monte Carlo methods become particularly impractical for large particle numbers $N$. Hence, a mean-field description is commonly used to simplify the analysis typically in excellent agreement with BEC experiments [26].

The theoretical foundation of the mean-field treatment was laid by Bogoliubov in 1947 [27]. The main idea is to separate the condensate contribution from the bosonic field operator, which is done in the following way. The individual single particle states $\phi_{k}$ form a basis so that the field operator can be expanded in terms 


\section{Bose-Einstein condensation}

of them

$$
\hat{\Psi}(\mathbf{r})=\sum_{k} \phi_{k}(\mathbf{r}) \hat{\alpha}_{k}
$$

with $\hat{\alpha}_{k}$ being the annihilation operator of state $k$ satisfying bosonic commutation relations. The ground state population $N_{0}$ in a Bose Einstein condensate is macroscopic meaning that the fraction $N_{0} / N$ remains finite in the thermodynamic limit $(N \rightarrow \infty)$ and therefore $N \sim N_{0} \gg 1$. As the action of an annihilation or creation operator is to change the state by adding or removing one particle the states $\left|N_{0}\right\rangle$ and $\left|N_{0}+1\right\rangle$ can be considered identical. Thus, the creation and annihilation operators can be treated as complex numbers, i.e. $\hat{\alpha}_{0}=\hat{\alpha}_{0}^{\dagger}=\sqrt{N_{0}}$ and the field operator is then decomposed into

$$
\hat{\Psi}(\mathbf{r})=\Psi(\mathbf{r})+\delta \hat{\Psi}(\mathbf{r}),
$$

where $\Psi=\langle\hat{\Psi}\rangle$ is the condensate wave function and $\delta \hat{\Psi}$ represents the noncondensed fraction of the particles which remains small in the BEC regime and can be treated perturbatively.

In order to develop an equation of motion for the condensate wave function let us start with the time evolution of the field operator $\hat{\Psi}(\mathbf{r}, t)$ which is given by the Heisenberg equations of motion $\mathrm{i} \hbar \frac{\partial \hat{\Psi}(\mathbf{r}, t)}{\partial t}=[\hat{\Psi}(\mathbf{r}, t), \hat{H}]$ yielding

$$
\begin{aligned}
\mathrm{i} \hbar \frac{\partial \hat{\Psi}(\mathbf{r}, t)}{\partial t}= & {\left[-\frac{\hbar^{2} \nabla^{2}}{2 M}+V_{\mathrm{tr}}(\mathbf{r})\right.} \\
& \left.+\frac{1}{2} \int d^{3} r^{\prime} \hat{\Psi}^{\dagger}\left(\mathbf{r}^{\prime}, t\right)\left[V_{\mathrm{int}}\left(\mathbf{r}-\mathbf{r}^{\prime}\right)+V_{\mathrm{int}}\left(\mathbf{r}^{\prime}-\mathbf{r}\right)\right] \hat{\Psi}(\mathbf{r}, t)\right] \hat{\Psi}(\mathbf{r}, t) .
\end{aligned}
$$

In the BEC regime, i.e. when most of the particles are Bose-Einstein condensed the non-condensed particles can be neglected in first order and the field operator $\hat{\Psi}$ can be replaced by the condensate wave function $\Psi$ which leads to the famous Gross-Pitaevskii equation (GPE)

$$
\mathrm{i} \hbar \frac{\partial \Psi(\mathbf{r}, t)}{\partial t}=\left(-\frac{\hbar^{2} \nabla^{2}}{2 M}+V_{\operatorname{tr}}(\mathbf{r})+g|\Psi(\mathbf{r}, t)|^{2}\right) \Psi(\mathbf{r}, t),
$$

which is a non-linear Schrödinger equation for the condensate wave function $\Psi$. The GPE was derived by E. P. Gross and L. P. Pitaevskii in 1961 [28, 29]. The equation is especially appealing since the complicated many-body problem is converted into solving a single differential equation for the complex-valued function $\Psi$.

Then the many-body wave function of the ground state $\Psi_{0}\left(\mathbf{r}_{1}, \mathbf{r}_{2}, \ldots, \mathbf{r}_{N}\right)$ of a $\mathrm{BEC}$ can be given in terms of the single particle ground state wave function $\varphi_{0}$

$$
\Psi_{0}\left(\mathbf{r}_{1}, \mathbf{r}_{r}, \ldots, \mathbf{r}_{N}\right)=\sqrt{N}\left(\varphi_{0}\left(\mathbf{r}_{1}\right) \varphi_{0}\left(\mathbf{r}_{2}\right) \ldots \varphi_{0}\left(\mathbf{r}_{N}\right)\right) .
$$

The ground state energy of the gas, $\left\langle\Psi_{0}|\hat{H}| \Psi_{0}\right\rangle$, is then given by the functional

$$
E\left[\varphi_{0}, \varphi_{0}^{*}\right]=N \int d^{3} r\left(\frac{\hbar^{2}}{2 M}\left|\nabla \varphi_{0}(\mathbf{r})\right|^{2}+V_{\operatorname{tr}}(\mathbf{r})\left|\varphi_{0}\right|^{2}+\frac{g}{2} N\left|\varphi_{0}(\mathbf{r})\right|^{4}\right) .
$$


In order to minimize the energy we have to deal with the constraint of particle conservation so that we introduce the Lagrange multiplier $\mu$. Then minimizing the new functional

$$
K\left[\varphi_{0}, \varphi_{0}^{*}\right]=E\left[\varphi_{0}, \varphi_{0}^{*}\right]-\mu N \int d^{3} r\left|\varphi_{0}(\mathbf{r})\right|^{2}
$$

with respect to $\varphi_{0}^{*}$ leads to

$$
\mu \varphi_{0}(\mathbf{r})=\left[-\frac{\hbar^{2} \nabla^{2}}{2 M}+V_{\mathrm{tr}}(\mathbf{r})+g N\left|\varphi_{0}(\mathbf{r})\right|^{2}\right] \varphi_{0} .
$$

In order to see this we multiply Eq. (1.16) by $\varphi_{0}^{*}$ and integrate over the whole position space and compare this with the derivative of the energy (1.14) with respect to the total particle number $N$. This shows that $\mu$ is indeed the chemical potential, i.e. $\mu=\frac{\partial E}{\partial N}$, and that the ground state evolves in time with respect to $\mu$

$$
\Psi_{0}(\mathbf{r}, t)=\varphi_{0}(\mathbf{r}) e^{-\mathrm{i} \mu t / \hbar}
$$

and not the energy of the state.

\subsection{Thomas-Fermi regime}

Let us discuss now an important limiting case in which the analysis of the GPE can be simplified if the density of the gas $n(\mathbf{r})=\left|\varphi_{0}(\mathbf{r})\right|^{2}$ changes slowly in space. Then the kinetic term can be neglected and the condensate is in the so-called Thomas-Fermi (TF) regime. For this let us introduce the typical length scale which is fixed by a balance of the kinetic and interaction term

$$
\frac{\hbar^{2}}{2 M \xi^{2}}=g n \quad \rightarrow \quad \xi=\frac{\hbar}{\sqrt{2 M g n}},
$$

which is called the healing length $\xi$. It is the minimal distance over which the condensate can adapt to any changes in the shape of the cloud. When the density variations over the length scale of $\xi$ are negligible small the kinetic energy contribution can be neglected and the GPE reduces to

$$
V_{\mathrm{tr}}(\mathbf{r})+g n(\mathbf{r})=\mu \text {. }
$$

The solution for the case of a BEC within a harmonic trap $V_{\operatorname{tr}}(\mathbf{r})=\frac{M}{2}\left(\omega_{x}^{2} x^{2}+\right.$ $\left.\omega_{y}^{2} y^{2}+\omega_{z}^{2} z^{2}\right)$, where $\omega_{i}$ is the trapping frequency in the $i$-direction, is given by

$$
\varphi_{0}(\mathbf{r})=\sqrt{\frac{\mu}{g}\left(1-\sum_{\eta=x, y, z} \frac{\eta^{2}}{R_{\eta}^{2}}\right)} \Theta\left(1-\sum_{\eta=x, y, z} \frac{\eta^{2}}{R_{\eta}^{2}}\right),
$$

where $R_{\eta}^{2}=\frac{2 \mu}{M \omega_{\eta}^{2}}$ and $\Theta$ is the Heaviside step function. Integrating over the density gives the total number of particles $N$ and the parameters $R_{\eta}$ and $\mu$ can be determined with the results $\mu=\frac{M}{2}\left(\frac{15 g N \omega_{x} \omega_{y} \omega_{z}}{4 \pi M}\right)^{2 / 5}, R_{x}=\left(\frac{15 g N \omega_{y} \omega_{z}}{4 \pi M \omega_{x}^{4}}\right)^{1 / 5}, R_{y}=$ $\omega_{x} R_{x} / \omega_{y}$, and $R_{z}=\omega_{x} R_{x} / \omega_{z}$. As the contact interaction is in the denominator in Eq. (1.18), stronger interactions (and therefore also larger number of particles) imply a smaller healing length. In many experiments the system is indeed in the Thomas-Fermi regime. 


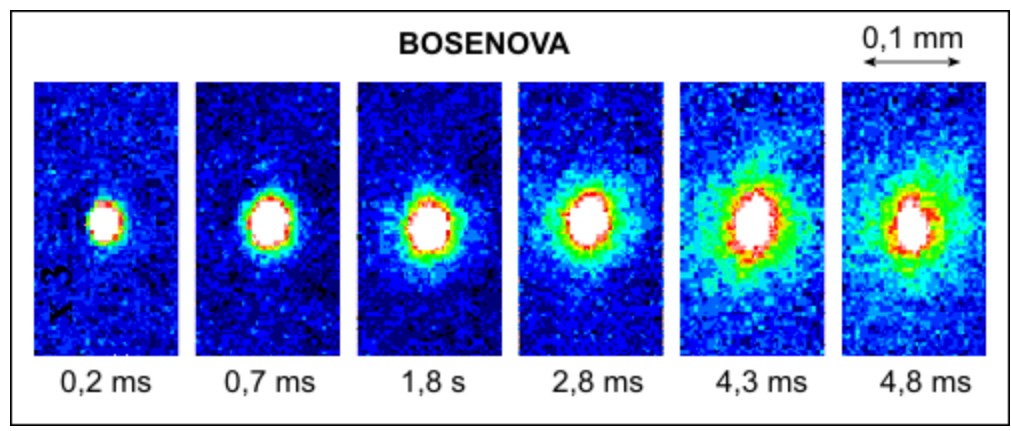

Figure 1.3.: Expansion of an atomic cloud of $1 \times 10^{4}{ }^{85} \mathrm{Rb}$ atoms after the collapse of a BEC (Bose-Nova). The picture was reprinted from Ref. [30].

\subsection{Stability and Collapse}

Another important question is the stability of a BEC, which is later in this Thesis very relevant in our discussion of dipolar BECs. As the kinetic energy exerts a repulsive quantum pressure, a non-interacting BEC is always stable. Let us restrict the discussion in this section to purely contact interacting systems. A free gas (without any confining potential) is unstable for an arbitrarily small attractive interaction whereas such a system with a repulsive interaction is obviously stable. Surprisingly, this situation changes qualitatively in a trap. For simplicity let us consider a harmonic trap and note that most of the stability properties in this trap geometry are qualitatively the same as in most other trapping geometries. The energy surface exhibits always a global minimum for repulsive interactions which is the stable solution. For negative scattering lengths, e.g. attractive contact interaction, the energy surface changes qualitatively. The global minimum is then always a collapsed state with zero volume. However for small enough interactions a second local minimum exists belonging to a metastable state. This local minimum represents a situation in which the repulsive quantum pressure compensates the attractive interaction. When the attractive interaction dominates the local minimum vanishes and the condensate does not have any (meta-)stable ground state. As the kinetic term scales linearly with the atom number $N$ in contrast to the squared dependence of the interacting term $N^{2}$, the number of atoms can have a severe influence on the stability of the condensate.

The first experiment on BEC collapse was done in 2001 [31] and revealed a surprising result. During the first stage of the collapse dynamics the width of the cloud decreases and the density increases in order to reduce the interaction energy reaching thereby large densities on the order of $n \sim 10^{23} \mathrm{~m}^{-3}$. Surprisingly, after reaching a maximal density the collapse stops and the condensate explodes afterwards [31] (see also Fig. (1.3)). This phenomena is called "Bose-Nova". After detailed investigation intrinsic inelastic processes, i.e. most crucially three-body interatomic collisions, were found to be the reason for the explosion. They become relevant at high densities when the distance between the particles becomes comparable to the characteristic range of the interatomic interactions leading to a local character [32]. Crucially, the three-body recombinations lead to atom loss, but do not destroy the phase coherence of the condensed atoms so that they can be incorporated by a loss term of the form $\left(-\frac{\mathrm{i} \hbar}{2} L_{3}|\Psi|^{4}\right) \Psi$ at the right-hand side 


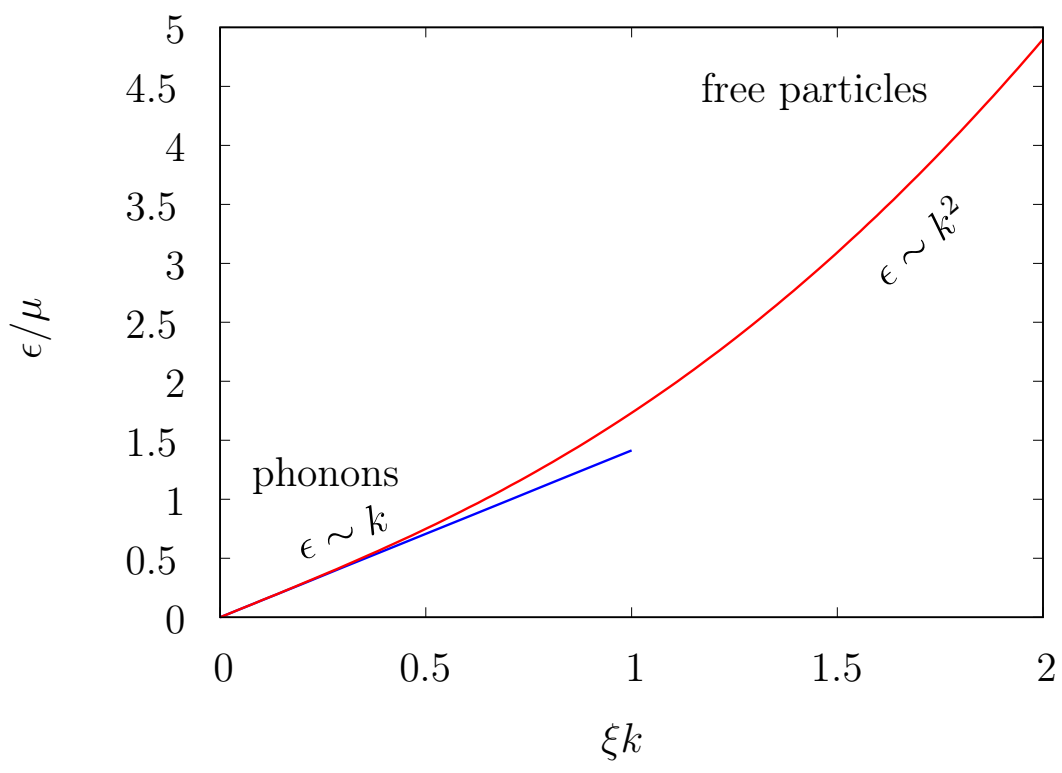

Figure 1.4.: Bogoliubov spectrum for a purely contact interacting homogeneous condensate. The spectrum changes it's behavior from linear for small momenta to quadratic for large momenta.

of the GPE (1.12), where $L_{3}$ is the three-body loss rate. We will employ such a treatment of the losses later in this thesis.

\subsection{Excitations}

A powerful tool to investigate the properties of a condensate is the study of the elementary excitations, which are small amplitude oscillations on top of the condensate wavefunction. They can be calculated by expanding the wave function $\Psi(\mathbf{r}, t)$ according to

$$
\Psi(\mathbf{r}, t)=[\Psi(\mathbf{r})+\vartheta(\mathbf{r}, t)] e^{-\mathrm{i} \mu t / \hbar},
$$

where $\vartheta$ is a small quantity of the form $\vartheta(\mathbf{r}, t)=\sum_{i} u_{i}(\mathbf{r}) e^{-\mathrm{i} \omega_{i} t}+v_{i}^{*}(\mathbf{r}) e^{\mathrm{i} \omega_{i} t}$ with $\omega_{i}$ being the frequency of the oscillations and $u_{i}$ and $v_{i}$ are the amplitudes. Inserting Eq. (1.21) into the GPE (1.12), considering only terms linear in $\vartheta$ and collecting all terms evolving in time like $e^{-\mathrm{i} \omega_{i} t}$ and $e^{\mathrm{i} \omega_{i} t}$ yields the Bogoliubov-de Gennes (BdG) equations [17]

$$
\begin{aligned}
\hbar \omega_{i} u_{i}(\mathbf{r}) & =\left[-\frac{\hbar^{2} \nabla^{2}}{2 M}+V_{\mathrm{tr}}(\mathbf{r})-\mu+2 g n(\mathbf{r})\right] u_{i}(\mathbf{r})+g(\Psi(\mathbf{r}))^{2} v_{i}(\mathbf{r}), \\
-\hbar \omega_{i} v_{i}(\mathbf{r}) & =\left[-\frac{\hbar^{2} \nabla^{2}}{2 M}+V_{\mathrm{tr}}(\mathbf{r})-\mu+2 g n(\mathbf{r})\right] v_{i}(\mathbf{r})+g\left(\Psi^{*}(\mathbf{r})\right)^{2} u_{i}(\mathbf{r}) .
\end{aligned}
$$

They enable the calculation of the eigenfrequencies $\omega_{i}$ as well as the amplitudes $u_{i}$ and $v_{i}$ of the excitation frequencies. In general, the $\mathrm{BdG}$ equations have to be solved numerically. However an analytical solution exhibiting the main physics of the excitations can be found in the case of a homogeneous condensate $\left(V_{\mathrm{tr}}(\mathbf{r})=0\right)$. Then the chemical potential is given by $\mu=g n$ and $\Psi$ is independent of the position, and can be chosen to be real $\Psi=\sqrt{n}$. The excitation frequencies 


\section{Bose-Einstein condensation}

can then be calculated by a Fourier transformation of these equations with the result [17]

$$
\epsilon(\mathbf{k})=\sqrt{\frac{\hbar^{2} \mathbf{k}^{2}}{2 M}\left(\frac{\hbar^{2} \mathbf{k}^{2}}{2 M}+2 g n\right)},
$$

where $\epsilon(\mathbf{k})=\hbar \omega_{\mathbf{k}}$. The solution is a continuous spectrum depending on the wave vector $\mathbf{k}$, which is a good quantum number in translational invariant systems. The spectrum is linear for small momenta $k$ such that $k^{2} \ll 2 / \xi^{2}$ and the excitations of this part of the spectrum are called phonons. For large momenta $\left(k^{2} \gg 2 / \xi^{2}\right)$ the spectrum is quadratic exhibiting a free particle dispersion relation.

There are also some important properties worth mentioning. First, real frequencies of the BdG equations correspond to small amplitude oscillations and complex frequencies signal a dynamical instability in which one of the amplitudes $(u$ or $v)$ increases exponentially. Second, for each solution $\left(u_{i}, v_{i}\right)$ with frequency $\omega_{i}$ exists always a solution $\left(u_{i}^{*}, v_{i}^{*}\right)$ with frequency $-\omega_{i}$. These two solutions correspond to the same physical oscillation. Third, $\omega_{i}=0$ is always a solution with $u=\alpha \Psi$ and $v=-\alpha \Psi$ which corresponds to a gauge transformation of the wave function $\Psi$ changing the complex phase and therefore does not describe a real physical excitation. Bogoliubov excitations will play a crucial role in this thesis. 


\section{Dipolar Bose-Einstein Condensates}

In the previous chapter we have briefly reviewed the basics of Bose-Einstein condensation as well as its mean-field description concentrating on the short-ranged contact interaction. This chapter is devoted to condensates with an additional dipole-dipole interaction (DDI) in which many qualitatively new phenomena can be observed due to the long-ranged and anisotropic nature of the DDI.

Dipole-dipole interactions can be found in a broad class of systems as they can be realized via electric or magnetic forces and are intensively studied in the context of highly magnetic atoms [33-35], polar molecules [36-48], and Rydberg gases [49-58]. The first study of a BEC with realistic dipole-dipole interaction was done in 2000 by K. Góral et al. [59] showing structured ground state solutions. At the same time important experimental progress in the trapping of atoms with higher magnetic moments $[60,61]$ was made and soon several theoretical papers investigated the ground state properties and the excitation spectrum of dipolar BECs [62-66]. The first Bose-Einstein condensate with significant dipole-dipole interaction was created in 2005 with ${ }^{52} \mathrm{Cr}$ atoms in the group of T. Pfau [33]. Thereby main properties of dipolar BECs could be investigated like the expansion [67,68], stability properties [69] and the d-wave collapse dynamics [70]. Since these first investigations the field of ultracold quantum gases with dipolar interactions has become a field of major interest. The production of dysprosium [34] and erbium BECs [35] has paved the way to condensates with even stronger dipolar interactions. Significant experimental progress was made by the group of B. Laburthe-Tolra studying thermodynamics and anisotropy effects [71-75]. Moreover, highly influential was the discovery of stable droplets by the group of $\mathrm{T}$. Pfau enabling to study the effects of quantum fluctuations [76-78]. On the theory side many theoretical proposals appeared since the initial studies including the investigation of ground states [79-83] and the Bogoliubov spectrum of dipolar BECs in different trapping geometries [84-89], the study of superfluidity [90,91], collapse dynamics [92-96], condensates in strongly rotating traps [97-101] as well as dipolar solitons [102-105]. More detailed and broader summaries can be found in the review articles [106-108].

In section 2.1 we discuss the properties of the dipole-dipole interaction followed by a study of the stability of a dipolar BEC in section 2.2. In section 2.3 we discuss the mean-field description in the presence of the dipolar interaction and ground state solutions. Section 2.4 is dedicated to the collapse dynamics and in section 2.5 we discuss the elementary excitations, focusing in section 2.6 on the study of the roton-maxon spectrum and the properties of rotonic excitations in dipolar Bose-Einstein condensates. 


\subsection{The dipole-dipole interaction}

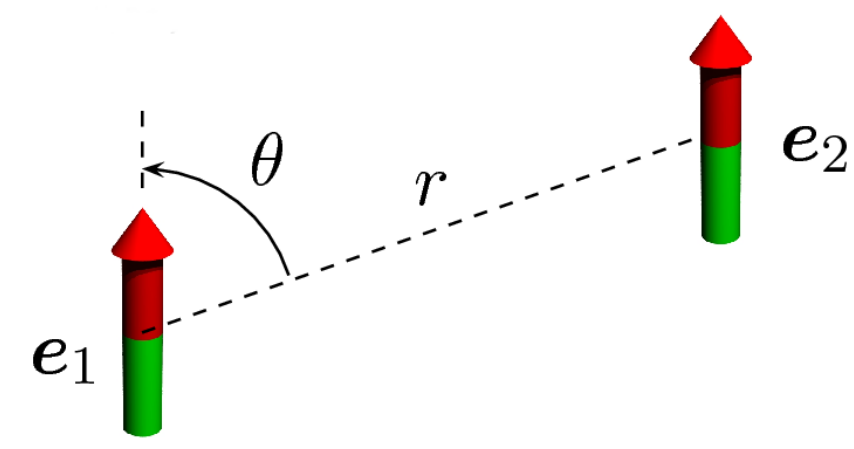

Figure 2.1.: Two polarized dipoles interacting with each other via the dipoledipole interaction separated by the distance $r$. The interaction strength depends on the angle $\theta$, leading to an attractive interaction for dipoles in a head-to tail configuration $(\theta=0)$ whereas dipoles sitting besides each other repel $(\theta=\pi / 2)$. The picture was reprinted from [107].

The dipole-dipole interaction potential between two particles with dipole moments oriented along the unit vectors $\mathbf{e}_{1}$ and $\mathbf{e}_{2}$ reads

$$
V_{\mathrm{dd}}(\mathbf{r})=\frac{C_{\mathrm{dd}}}{4 \pi} \frac{\left(\mathbf{e}_{1} \cdot \mathbf{e}_{2}\right) r^{2}-3\left(\mathbf{e}_{1} \cdot \mathbf{r}\right)\left(\mathbf{e}_{2} \cdot \mathbf{r}\right)}{r^{5}},
$$

where $\mathbf{r}$ is the position vector from particle 1 to 2 (see Fig. (2.1)) and its absolute value is given by $r=|\mathbf{r}|$. The constant $C_{\mathrm{dd}}$ is the dipolar interaction strength which takes the form $C_{\mathrm{dd}}=d^{2} / \epsilon_{0}$ in the case of electric dipoles, where $d$ is the electric dipole moment and $\epsilon_{0}$ the vacuum permitivity. In the case of magnetic dipoles the coupling strength is given by $C_{\mathrm{dd}}=\mu_{0} \mu^{2}$, where $\mu_{0}$ is the vacuum permeability and $\mu$ the magnetic dipole moment. Usually, dipolar interactions are much stronger in the electric case as $\frac{\mu_{0} \mu^{2}}{d^{2} / \epsilon_{0}} \sim \alpha^{2} \sim 10^{-4}$, where $\alpha$ is the fine structure constant. The expression for the dipole-dipole potential (2.1) simplifies when the dipoles are polarized by an external electric or magnetic field leading to

$$
V_{\mathrm{dd}}(\mathbf{r})=\frac{C_{\mathrm{dd}}}{4 \pi} \frac{1-3 \cos ^{2} \theta}{r^{3}}
$$

where $\theta$ is the angle between the direction of the dipoles and the relative position of the particles $\mathbf{r}$. We align our coordinate system such that the dipole orientation corresponds to the $z$-direction. The dipole-dipole interaction is anisotropic as it is attractive between two dipoles placed in a head-to tail configuration $(\theta=0)$, repulsive if they are besides each other $(\theta=\pi / 2)$, and vanishing at the magic angle $\left(\theta_{m}=\arccos (1 / \sqrt{3}) \sim 54,7\right)$. Additionally, the dipole-dipole interaction is long-ranged as it decays with a power law of $r^{-3}$. A more detailed discussion about the definition of long-ranged and short-ranged interactions can be found in [107] and references therein. 
The dipole-dipole interaction is tunable by applying a rotating polarizing field. The time-averaged interaction between rotating dipoles can be significantly smaller than interaction between non-rotating ones and even change its sign (Fig. $(2.2)$ ), where at the same time the functional form of the potential is retained. For definiteness consider the case of magnetic dipoles in the time-dependent magnetic field $\mathbf{B}=B \mathbf{e}(t)$, where $\mathbf{e}(t)=\cos \varphi \mathbf{e}_{z}+\sin \varphi\left[\cos (\Omega t) \mathbf{e}_{x}+\sin (\Omega t) \mathbf{e}_{y}\right]$ characterizes a rotation of the dipoles around the $z$-axis around a cone of angle $2 \varphi$ with an angular frequency $\Omega$. When the rotation frequency $\Omega$ is much smaller than the Larmor frequency $\mu B / \hbar$, but much larger than the trapping frequencies $\omega_{i}$, the time averaged potential reads [107]

$$
\left\langle V_{\mathrm{dd}}(\mathbf{r}, t)\right\rangle=\frac{C_{\mathrm{dd}}}{4 \pi} \frac{1-3 \cos ^{2} \theta}{r^{3}}\left[\frac{3 \cos ^{2} \varphi-1}{2}\right] .
$$

The last factor in the brackets varies from 1 to $-1 / 2$ depending on the tilting angle $\varphi$. The time-averaged potential $\left\langle V_{\mathrm{dd}}(\mathbf{r}, t)\right\rangle$ is zero for the magic angle $\varphi_{m}=$ $\arccos (1 / \sqrt{3})$ and the interaction strength changes its sign for $\varphi>\varphi_{m}$, which enables to study an otherwise not physically accessible situation.

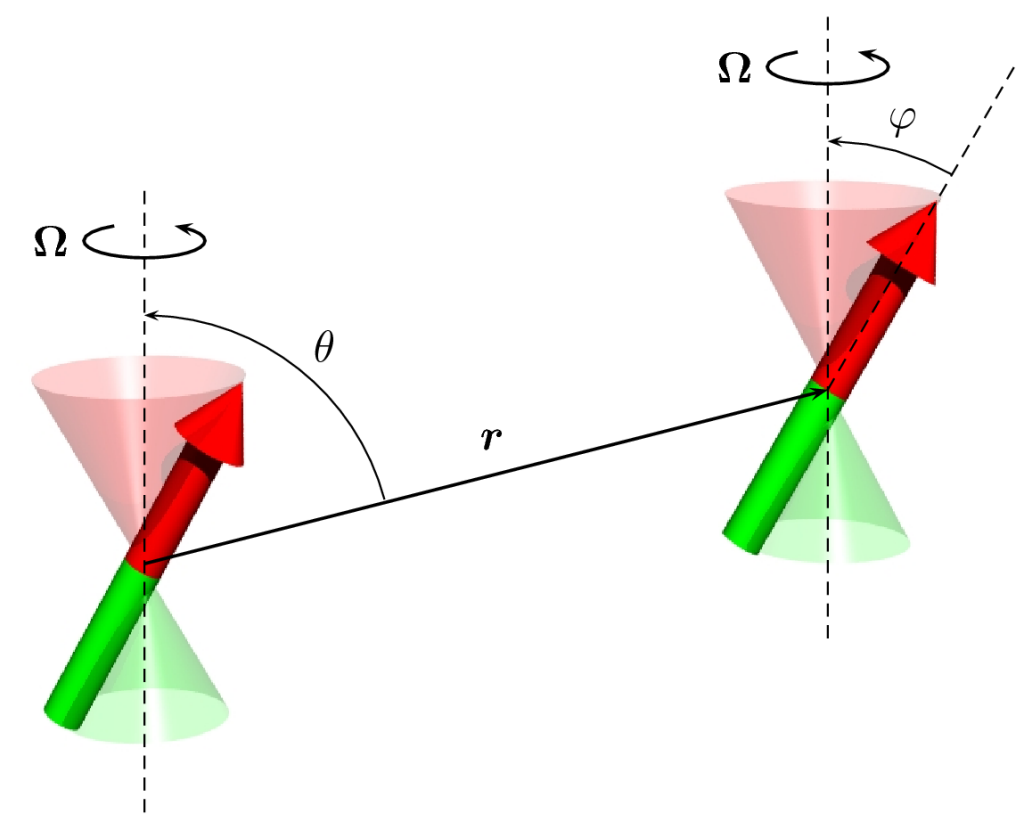

Figure 2.2.: Tuning of the dipole-dipole interaction by precessing the dipoles around the $z$-direction on a cone of angle $2 \varphi$. The picture was reprinted from [107].

The Fourier transformed dipole-dipole potential is used in most of the calculations involving the dipolar interaction. Therefore, its form is given here. The Fourier integrals can be calculated by expanding the exponential factor $e^{-\mathrm{ir} \cdot \mathbf{k}}$ in terms of spherical harmonics and using the fact that the angular dependence of the dipole-dipole potential can be expressed in terms of the spherical harmonic 
function $-4 \sqrt{\frac{\pi}{5}} Y_{2}^{0}(\vartheta, \varphi)=\left(1-3 \cos ^{2} \vartheta\right)$, leading to the result [107]

$$
\begin{aligned}
\tilde{V}_{\mathrm{dd}}(\mathbf{k}) & =\int d^{3} r e^{-\mathrm{i} r \cdot \mathbf{k}} V_{\mathrm{dd}}(\mathbf{r}), \\
& =\frac{C_{\mathrm{dd}}}{3}\left(3 \cos ^{2} \theta_{k}-1\right),
\end{aligned}
$$

where $\theta_{k}$ is the angle between the direction of the dipoles and the relative vector between the dipoles in Fourier space. Unlike the dipole-dipole potential in position space the Fourier transform $\tilde{V}_{\text {dd }}$ does not diverge or posses any singular points, but still exhibits a non-continuous behavior at the origin.

\subsection{Geometrical Stability}
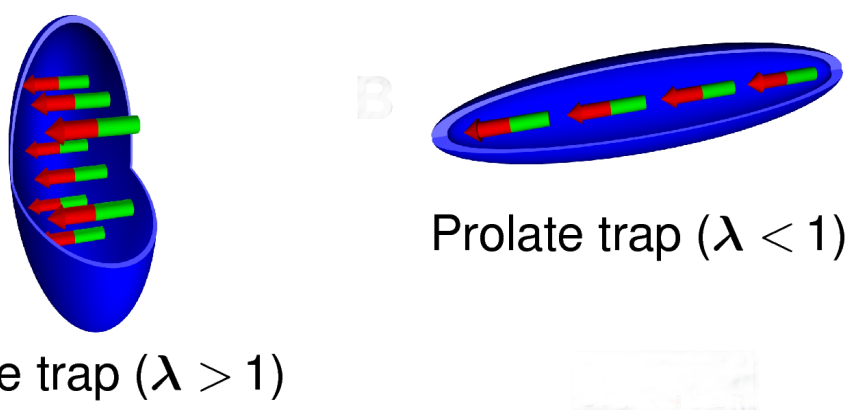

Oblate trap $(\lambda>1)$

Figure 2.3.: Intuitive picture of the geometrical stability of a dipolar BEC. In a pancake (oblate) geometry the dipoles are mostly in a side-by side position, so that the DDI is on average repulsive. On the contrary the DDI is on average attractive when the dipoles are in a head-to tail configuration. Therefore pancake-shaped BECs are inherently more stable. The picture was reprinted from [69].

The trapping potential determines crucially the shape of the condensate in a typical experiment. Therefore, due to the anisotropic nature of the DDI the stability of dipolar BECs depends strongly on the trapping geometry. The dipoles are mostly in a side-by side position in a pancake trap resulting in a dipoledipole interaction which is on average repulsive (see Fig. (2.3)). On the other hand the dipoles are mostly in a head-to-tail configuration in a cigar-shaped geometry leading to an on average attractive DDI. This geometrical stability was experimentally investigated in 2008 by the group of T. Pfau [69]. The experimental sequence was as follows. First a condensate of ${ }^{52} \mathrm{Cr}$ atoms with dominant repulsive contact interaction was produced in a cylindrically harmonic trap $V_{\mathrm{tr}}=\frac{M}{2} \omega_{\mathrm{r}}^{2}\left(x^{2}+y^{2}\right)+\frac{M}{2} \omega_{z}^{2} z^{2}$. Afterwards, the contact interaction strength was reduced by means of a Feshbach resonance until the condensate gets unstable at a critical scattering length $a_{\text {crit }}$. The geometry of the trap is characterized by the trap aspect ratio $\lambda=\omega_{z} / \omega_{\mathrm{r}}$ which fulfills $\lambda>1$ for pancake geometries and $\lambda<1$ for cigar geometries. The dependence of the critical scattering length on the trapping geometry can be seen in Fig. (2.4). The green curve marks the 
border of instability, which is a decreasing function of $\lambda$, showing that the condensate gets more stable if the trap is more pancake-like. However in highly cigar or pancake traps the dependence is very weak. The condensate is stable regardless of the trapping geometry if the scattering length is bigger than the dipole length $a_{\mathrm{dd}}=\frac{C_{\mathrm{dd}} M}{12 \pi \hbar^{2}}$. Then the contact interaction dominates and the ground state corresponds to a global minimum in the energy surface. For small scattering lengths below $-2 a_{\mathrm{dd}}$ the condensate is unstable again irrespective of the trapping geometry. The ground state in this case is a collapsed state with the shape of an infinitely thin cylinder. Between these two regions the stability depends on the geometry. In particular for $\lambda \gtrsim 1$ the ground state is a collapsed state, but an additional metastable solution exists corresponding to a local minimum of the energy surface, which vanishes at the border of instability.

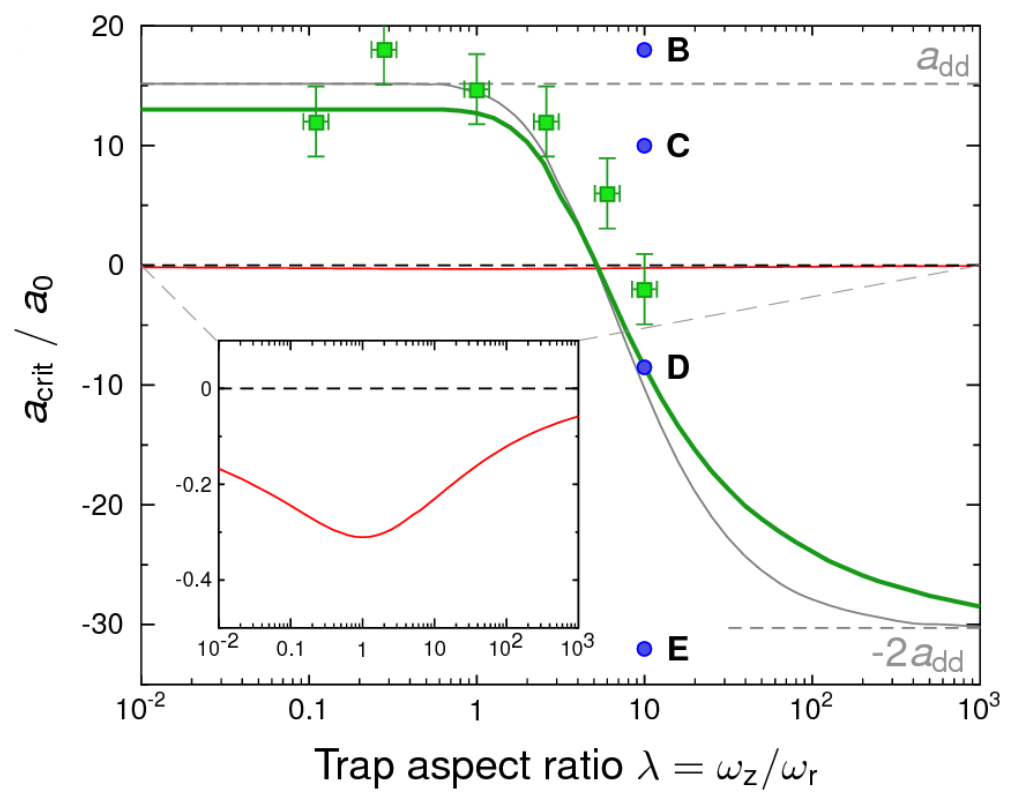

Figure 2.4.: Stability diagram of a condensate of ${ }^{52} \mathrm{Cr}$ atoms in a cylindersymmetric harmonic trap with $\lambda=\omega_{z} / \omega_{\mathrm{r}}$. At the point B the energy surface exhibits a global minimum corresponding to a stable solution. At the points $\mathrm{C}, \mathrm{D}$ the global minimum is a collapsed state, but an additional metastable solution corresponding to a local minimum in the energy surface exists. At the point E no stable solution exists. The picture was reprinted from [69].

\subsection{Mean-field description and ground state properties}

A mean-field description for a dipolar BEC can be derived in an analogous way to the purely contact case. The theoretical foundation was laid by You and Yi $[62,63]$ who constructed a pseudo-potential for a general anisotropic interaction. This result can be used to derive a GPE including the dipole-dipole interaction via an 
additional term yielding

$$
\mathrm{i} \hbar \frac{\partial \Psi(\mathbf{r}, t)}{\partial t}=\left[-\frac{\hbar^{2} \nabla^{2}}{2 M}+V_{\mathrm{tr}}(\mathbf{r})+g n(\mathbf{r}, t)+\int d^{3} r^{\prime} V_{\mathrm{dd}}\left(\mathbf{r}-\mathbf{r}^{\prime}\right) n\left(\mathbf{r}^{\prime}, t\right)\right] .
$$

The GPE becomes an integro-differential equation for the condensate wave function $\Psi$. The dipolar pseudo-potential contains also a contact part proportional to $\delta(\mathbf{r})$ which is absorbed into the regular contact term making $g=g\left(C_{\mathrm{dd}}\right)$ dependent on the dipole strength. The validity of Eq. (2.5) has been studied by means of Monte-Carlo simulations [92,109,110] showing an excellent agreement, provided the gas is in the dilute regime, $n a_{s}^{3} \ll 1$ and far from shape resonances.

With the nonlocal GPE at hand the ground state properties of a dipolar BEC can be studied. An analytical solution has been derived in the Thomas-Fermi regime $[111,112]$ within a cylindrically symmetric trap with the surprising result that the ground state density profile has an inverted paraboloid shape $n(\mathbf{r})=$ $n_{0}\left(1-\frac{r^{2}}{R_{\rho}^{2}}-\frac{z^{2}}{R_{z}^{2}}\right) \Theta\left(1-\frac{r^{2}}{R_{\rho}^{2}}-\frac{z^{2}}{R_{z}^{2}}\right)$ as in the case of a purely contact interacting gas, but with different Thomas-Fermi radii $R_{i}$.

However, full numerical simulations of the GPE (2.5) revealed structured ground state solutions close to the boundary of instability [59]. Especially it was shown in $[85,86]$ that anomalous density profiles in which the maximal density is not at the trap center, but on a ring around it, exist in dipolar BECs. These cloud shapes can only be found close to instability at certain trap aspect ratios, forming an island structure in the $C_{\mathrm{dd}^{-}} \lambda$ space.

\subsection{Collapse dynamics in Chromium}

As shown in section 1.5 the collapse dynamics of purely contact interacting BECs revealed the astonishing result of an explosion of the condensate after the initial implosion [31]. Condensates with dipolar interactions reveal again surprising results as was shown in the first experimental study of a dipolar collapse in 2008 [70]. The experimental procedure was at follows. First a stable BEC of chromium atoms was created at a scattering length bigger than the critical value $a_{\text {crit }}$. Afterwards the condensate was destabilized by reducing the scattering length using a magnetic Feshbach resonance. The system evolved for an adjustable time after which the trap was switched off. The cloud was then imaged by absorption spectroscopy after the time-of flight, which can be seen in Fig. (2.5). During the collapse the width of the condensate reduces in the direction perpendicular to the dipole direction and the density increases. This originates directly from the anisotropic nature of the DDI as the collapsed mean-field state is an infinitely thin cylinder. The chromium condensate collapses until inelastic threebody losses dominate at large densities which lead to large particle losses and drive the following explosion. As the condensate mainly collapsed perpendicular to the dipole-direction the cloud expands also mainly in this direction leading to an anisotropic expansion. In particular the post collapse symmetry of the condensate is reminiscent of the d-wave angular symmetry of the dipole-dipole interaction.

However, a fragmentation of the condensate during the collapse followed by a stabilization of these single quantum droplets were shown in condensates with stronger DDI [76-78]. We discuss this phenomenon in detail in chapter 5 . 


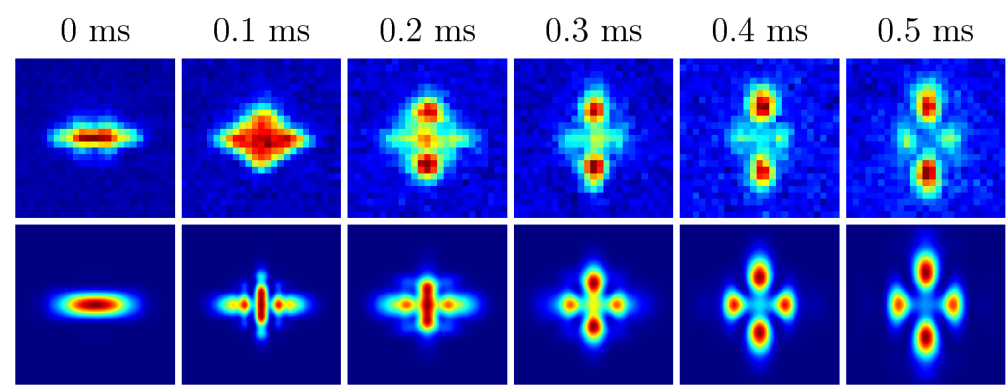

Figure 2.5.: Experimental images of the d-wave collapse dynamics in a condensate of ${ }^{52} \mathrm{Cr}$ atoms and numerical simulations of Eq. (2.5) expanded by a three-body loss term of the form $\left(-\mathrm{i} \frac{\hbar L_{3}}{2} n^{2}\right) \Psi$. The upper series of pictures are taken from absorption spectroscopy of different experimental runs and the lower pictures are theoretical results from solving Eq. (2.5), showing an excellent agreement with the experiment. The picture was reprinted from [70].

\subsection{Excitations}

The elementary excitations can be calculated by inserting the Bogoliubov ansatz for the condensate wave function $\Psi$ (Eq. 1.21) into the GPE leading to

$$
\begin{array}{r}
{\left[-\frac{\hbar^{2} \nabla^{2}}{2 M}+V_{\mathrm{tr}}(\mathbf{r})+2 g n(\mathbf{r})+\int d^{3} r^{\prime} V_{\mathrm{dd}}\left(\mathbf{r}-\mathbf{r}^{\prime}\right) n\left(\mathbf{r}^{\prime}\right)-\mu\right] u_{i}(\mathbf{r})+g \Psi(\mathbf{r})^{2} v_{i}(\mathbf{r})} \\
+\Psi(\mathbf{r}) \int d^{3} r^{\prime} V_{\mathrm{dd}}\left(\mathbf{r}-\mathbf{r}^{\prime}\right)\left[\Psi\left(\mathbf{r}^{\prime}\right) v_{i}\left(\mathbf{r}^{\prime}\right)+\Psi^{*}\left(\mathbf{r}^{\prime}\right) u_{i}\left(\mathbf{r}^{\prime}\right)\right]=\hbar \omega_{i} u_{i}(\mathbf{r}) \\
{\left[-\frac{\hbar^{2} \nabla^{2}}{2 M}+V_{\mathrm{tr}}(\mathbf{r})+2 g n(\mathbf{r})+\int d^{3} r^{\prime} V_{\mathrm{dd}}\left(\mathbf{r}-\mathbf{r}^{\prime}\right) n\left(\mathbf{r}^{\prime}\right)-\mu\right] v_{i}(\mathbf{r})+g\left(\Psi^{*}(\mathbf{r})\right)^{2} u_{i}(\mathbf{r})} \\
+\Psi^{*}(\mathbf{r}) \int d^{3} r^{\prime} V_{\mathrm{dd}}\left(\mathbf{r}-\mathbf{r}^{\prime}\right)\left[\Psi^{*}\left(\mathbf{r}^{\prime}\right) u_{i}\left(\mathbf{r}^{\prime}\right)+\Psi\left(\mathbf{r}^{\prime}\right) v_{i}\left(\mathbf{r}^{\prime}\right)\right]=-\hbar \omega_{i} v_{i}(\mathbf{r}) .
\end{array}
$$

In the case of a homogeneous system the spectrum can be calculated analytically by Fourier transforming the equations leading to

$$
\epsilon(\mathbf{k})=\sqrt{\frac{\hbar^{2} k^{2}}{2 M}\left(\frac{\hbar^{2} k^{2}}{2 M}+2 g n\left(1+\epsilon_{\mathrm{dd}}\left(3 \cos ^{2} \theta-1\right)\right)\right)},
$$

where $\epsilon_{\mathrm{dd}}=\frac{C_{\mathrm{dd}}}{3 g}$ characterizes the strength of the dipole-dipole interaction relative to the contact interaction. The spectrum is anisotropic due to the presence of the DDI and contains again a linear phononic part for small $k$. The energy of modes with $\theta<\arccos \left[\frac{1}{\sqrt{3}}\right]$ increases when $\epsilon_{\mathrm{dd}}$ is increased and are hence called hard modes. On the contrary the energy of modes with $\theta>\arccos \left[\frac{1}{\sqrt{3}}\right]$ decreases for increasing $\epsilon_{\mathrm{dd}}$ and are thus called soft modes. An intuitive picture why modes with momentum perpendicular to the dipole direction softens can be seen in Fig. (2.6). From Eq. (2.7) it can be seen that the condensate becomes dynamically unstable for $\epsilon_{\mathrm{dd}}>1$. 
(a)

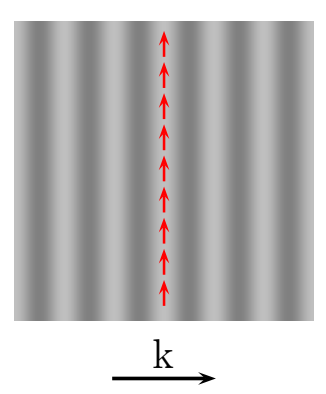

(b)

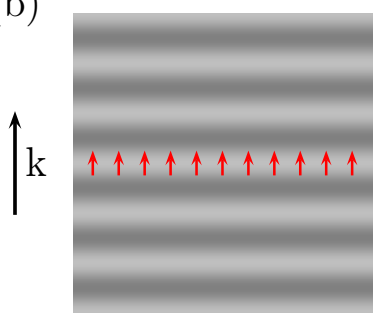

Figure 2.6.: Two excitations with $\mathbf{k}$ perpendicular to the dipole direction (a) and parallel to it (b). In the former case planes of high density are created in the direction of the dipoles and therefore the energy of the excitations is reduced. The opposite happens for the case b) leading to a hardening of the modes. The picture was reprinted from Ref. [107].

Additionally, analytical formulas for several low-energy excitations has been derived in the Thomas-Fermi regime $[66,111]$ as well as by a Gaussian ansatz $[62$, $63,65]$ of the form

$$
\Psi(x, y, z, t)=A(t) \prod_{\eta=x, y, z} e^{-\frac{\left[\eta-\eta_{0}(t)\right]^{2}}{2 w_{\eta}^{2}(t)}+\mathrm{i} \eta \alpha_{\eta}(t)+\mathrm{i} \eta^{2} \beta_{\eta}(t)}
$$

where $A(t), \eta_{0}(t), w_{\eta}(t), \alpha_{\eta}(t)$, and $\beta_{\eta}(t)$ are time-dependent variational parameters determined by minimizing the total energy of the condensate. The calculations revealed the surprising result that the character of the mode causing the instability changes its character depending on the trapping geometry. In a purely contact interacting condensate the most unstable mode causes a simultaneous expansion and contraction of the condensate in all directions, the so-called monopole mode. In the dipolar case the mode causing the collapse is either a monopole-like mode for $(\lambda<1)$ or a quadrupole like mode for $\lambda>1$ in which the directions perpendicular and parallel expand and contract oppositely.

In the case of a non-homogeneous system Ronen et al. calculated the elementary excitations of a condensate within a cylindrically symmetric harmonic trap numerically [84]. Additionally a variational approach has been derived in [113] giving a good approximation for the energetically lowest excitations.

\subsection{Roton Excitations}

In 1938 Kapitza, Allen and Misener found the phenomenon of superfluidity in liquid helium $[114,115]$ which is characterized by a non-viscous flow and the frictionless motion of an impurity below a critical velocity $v_{\max }$ [116]. L. D. Landau showed in a series of papers [117-119] that the explanation of the superfluid experiments demanded a non-monotonic excitation spectrum. This proposed spectrum should be linear for small momenta, eventually reaching a local maximum, followed by a decrease in the spectrum until reaching a local minimum at a finite momentum. The line $\epsilon(\mathbf{k})=\hbar v_{\max }$ becomes tangent to the dispersion curve close to the minimum. Landau suggested that this minimum originates from a 
rotation-like excitation and hence the name roton minimum [120]. In 1956 Feynman and Cohen made a big step forward in the understanding of the phenomenon when they identified the relation between the static structure factor $S(\mathbf{k})$ and the collective modes dispersion [121-123]

$$
\epsilon_{k}=\frac{E_{k}}{S(\mathbf{k})}
$$

In these works the strong correlations in liquid helium were found to be the origin of the roton-minimum as the static structure factor is related to the Fourier transform of the pair correlation function [124]. Soon afterwards experiments with neutron scattering confirmed the existence of the roton-minimum in liquid helium at $k_{\min }=1.93 \AA^{-1}[125-128]$.
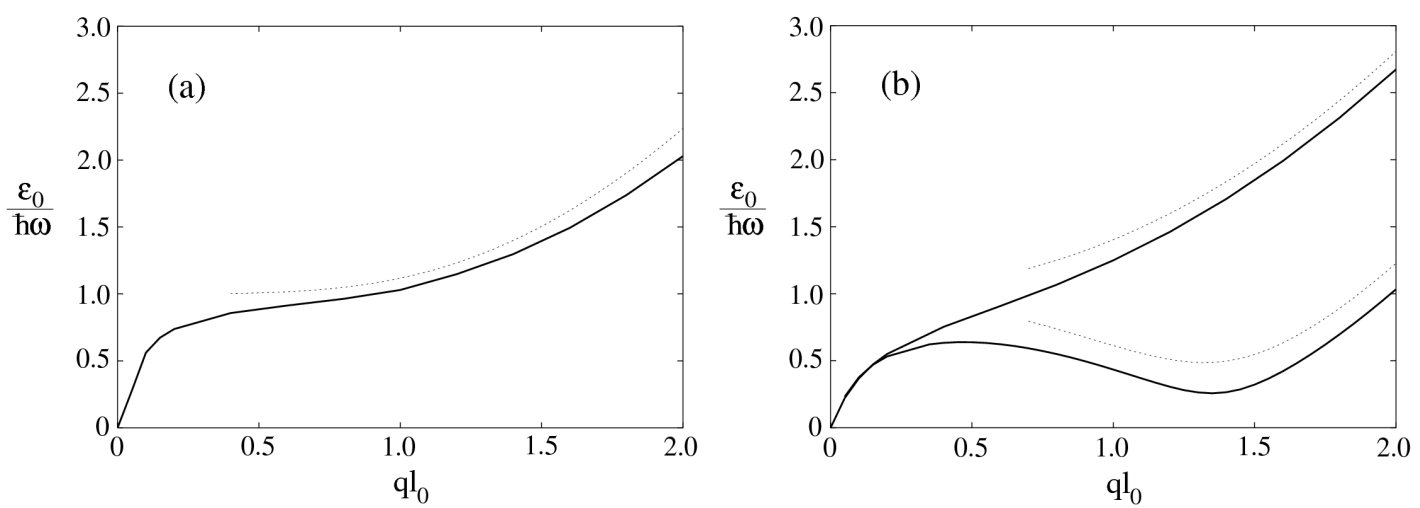

Figure 2.7.: Dispersion relation of a dipolar BEC trapped in the dipole direction and infinite perpendicular to it. The left figure shows a monotonous spectrum for $\epsilon_{\mathrm{dd}}=1$. The lower curve in the right figure shows for $\epsilon_{\mathrm{dd}}>1$ the roton-maxon spectrum whereas no minimum appears for $\epsilon_{\mathrm{dd}}<1$. The solid curves show numerical results and the dotted lines the results of Eq. (2.10). The picture was reprinted from [66].

In 2003 Santos et al. [66] showed that dipolar BECs can also exhibit a nonmonotonic spectrum containing a local maximum and minimum at finite momentum. They considered a dipolar BEC which is trapped in the dipole direction and infinite perpendicular to it. The in-plane momentum q can be used as quantum number as the system is translational invariant in the plane perpendicular to the dipole direction. Consider $R$ to be the typical size of the condensate in the direction of the dipoles. Then for low momenta such that $q R \ll 1$ the excitations are basically two-dimensional. The dipoles sit in a side-by-side configuration and repel each other so that the excitation spectrum is linear in this regime and the elementary excitations are phonons. However, for larger momenta $q R \gg 1$ the excitations aquire a three-dimensional character. As the dipole-dipole interaction is attractive in the third direction the repulsive character of the total interaction is reduced, which leads to an energy decrease for increasing in-plane momentum $q$. For even larger momenta the kinetic term dominates and the spectrum coincides with the typical free-particle solution. As this excitation spectrum resembles the one found in helium the excitations around the minimum are also called rotons. However, the origin of the roton minimum in dipolar BECs stems 
from the anisotropic nature of the interactions and not from the static structure factor as in helium.

Santos et al. derived an analytical expression of the excitation spectrum for large momenta $q R \gg 1$ so that the excitations have a three-dimensional character which reads [66]

$$
\epsilon(\mathbf{q})^{2}=E_{q}^{2}+\frac{4\left(1-\epsilon_{\mathrm{dd}}\right)\left(5 \epsilon_{\mathrm{dd}}+1\right)}{3\left(2 \epsilon_{\mathrm{dd}}+1\right)\left(4 \epsilon_{\mathrm{dd}}+1\right)} E_{q} \mu+\hbar^{2} \omega^{2},
$$

with $E_{q}=\frac{\hbar^{2} q^{2}}{2 M}$, and $\omega$ being the trapping frequency in the direction of the dipoles. Eq. (2.10) gives two types of behavior for the spectrum. For $\epsilon_{\mathrm{dd}}<1$ the energy increases monotonously, whereas for $\epsilon_{\mathrm{dd}}>1$ the dispersion law exhibits a local minimum. An important conclusion from Eq. (2.10) is that the depth of the minimum depends crucially on the chemical potential $\mu$ and thereby on the density. Hence the energy of the minimum decreases for increasing density until it reaches $\epsilon_{q}=0$ at approximately $q=\sqrt{2} / l_{0}$, where $l_{0}=\sqrt{\frac{\hbar}{M \omega}}$ is the characteristic length of the trapping potential. At this point the condensate becomes dynamically unstable against rotonic excitations. In a condensate with normal density profile, i.e. the maximal density is at the trap center, the mode causing the collapse has zero projection of angular momentum $m=0$ leading to an angular symmetric modulational instability. However, as discussed in the previous sections dipolar condensates can also exhibit density shapes in which the peak density lies on a ring around the trap center. BECs with such a "bloodcell" structure are destabilized by a mode with nonzero projection of angular momentum $m \neq 0$, leading to a so-called angular roton instability [94]. During this collapse the density distortion increases until peaks emerge along the ring which individually collapse. Thereby the number of peaks reveals the projection of the angular momentum of the mode causing the collapse [84-86].
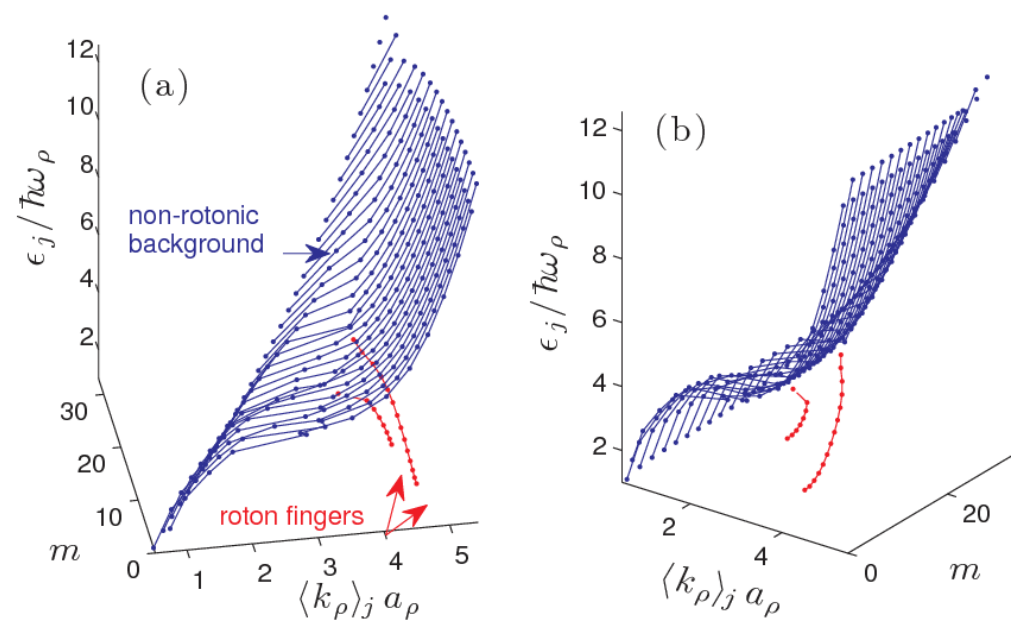

Figure 2.8.: Two views of the excitation spectrum of a dipolar BEC in a cylindrically harmonic trap. Blue dots depict the non-rotonic background and red dots the rotonic excitations forming finger like structures. The picture was reprinted from [88]. 
Additionally, full numerical simulations of the Bogoliubov spectrum in a cylinder symmetric harmonic trap were performed in 2013 by Bisset et al. [88]. The energy of the individual modes was calculated and depicted against their projection of angular momentum and an effective linear momentum $k$ (see [88] for the definition and more details) which can be seen in Fig. (2.8). Most of the excitations form a plane which is called the non-rotonic background with a linear behavior for small $k$. However, several excitations at intermediate momentum lie significantly below this plane and are rotonic modes. Because of their distinct position and form these structures are called "roton fingers".

This analysis was extended to the case of a general anisotropic trapping potential [129] and highly anharmonic traps [130]. The numerical results of Ref. [88] confirmed the so-called roton confinement discussed in Ref. [89]. Roton confinement results from the inhomogeneous density profile, which leads to a spatial dependence of the roton minimum (using local density approximation and Eq. (2.10)). Rotons are hence energetically confined at the trap center leading to the discrete roton fingers.

Moreover, several proposals to measure the roton-maxon spectrum or directly detecting the minimum at intermediate momentum have been derived in the last years, as using Bragg-spectroscopy [87], anomalous atom number fluctuations [131] or stability spectroscopy $[132,133]$. However, until now no direct measurement of rotonic excitations in dipolar BEC has been performed. 


\section{Phonon-to-roton crossover}

As explained in chapter 2 the stability of a dipolar BEC depends strongly on geometry and the energy of an excitation mode approaches zero when the condensate becomes dynamically unstable. A BEC confined in a highly pancake shaped trap exhibits a roton-maxon spectrum in its Bogoliubov dispersion and the most unstable mode is always a rotonic one. On the other hand for cigar-shaped and spherical traps the most unstable mode belongs to the phononic branch. Hence, the condensate can undergo rotonic or phononic collapses, depending crucially on the trapping geometry. We study the properties of the most unstable mode all the way from spherical to largely pancake-shaped traps revealing a crossover from a phonon to a roton unstable system in which the mode causing the collapse becomes increasingly local.

In section 3.1 we derive the stability diagram for a dysprosium condensate. Section 3.2 is dedicated to the analysis of the excitation spectrum for different trapping geometries. In section 3.3 we study the collapse dynamics of normal and bloodcell condensates and conclude in section 3.4.

\subsection{Stability diagram}

In order to study the condensate close to instability we first have to map the stability boundary. We choose as system parameters values which are accessible in current experiments considering a condensate of $N=20000$ dysprosium atoms in a cylindrically symmetric harmonic trap $V_{\mathrm{tr}}(\mathbf{r})=\frac{M}{2} \omega_{r}^{2}\left(x^{2}+y^{2}\right)+\frac{M}{2} \omega_{z}^{2} z^{2}$ with fixed geometric mean frequency $\tilde{\omega}=\left(\omega_{r}^{2} \omega_{z}\right)^{1 / 3}=70 \cdot 2 \pi s^{-1}$. Then, the trap aspect ratio $\lambda=\omega_{z} / \omega_{r}$ uniquely defines the trapping potential. First we calculate the ground state wave function $\Psi$ by solving the time independent GPE

$$
0=\left[-\frac{\hbar^{2} \nabla^{2}}{2 M}+V_{\mathrm{tr}}(\mathbf{r})+g n(\mathbf{r})+\int d^{3} r^{\prime} V_{\mathrm{dd}}\left(\mathbf{r}-\mathbf{r}^{\prime}\right) n\left(\mathbf{r}^{\prime}\right)-\mu\right] \Psi(\mathbf{r}) .
$$

To this end, we represent $\Psi$ on a three-dimensional grid and evolve an initial wave function in imaginary time $\tau=\mathrm{it}$, where we normalize the density after each time step. Then, the wave function converges to a local minimum of the energy surface $^{1}$. We employ for the time evolution the split operator technique [134] in which the time evolution operator in imaginary time is given by

$$
e^{-\frac{\hat{H} \Delta \tau}{\hbar}}=e^{-\frac{\hat{T} \Delta \tau}{\hbar}} e^{-\frac{\hat{V} \Delta \tau}{\hbar}}+O\left(\Delta \tau^{2}\right)
$$

\footnotetext{
${ }^{1}$ For scattering lengths $a_{s} \gtrsim a_{\mathrm{dd}}$ only a single global minimum exists which is then the solution of the imaginary time evolution. For trap aspect ratios $\lambda \gtrsim 2$ and $a_{s} \lesssim a_{\mathrm{dd}}$ an additional metastable solution can be found with the true ground state being a collapsed one (see also section 2.2). We choose an extended initial wave function in order to find the metastable solution as long as it exists.
} 


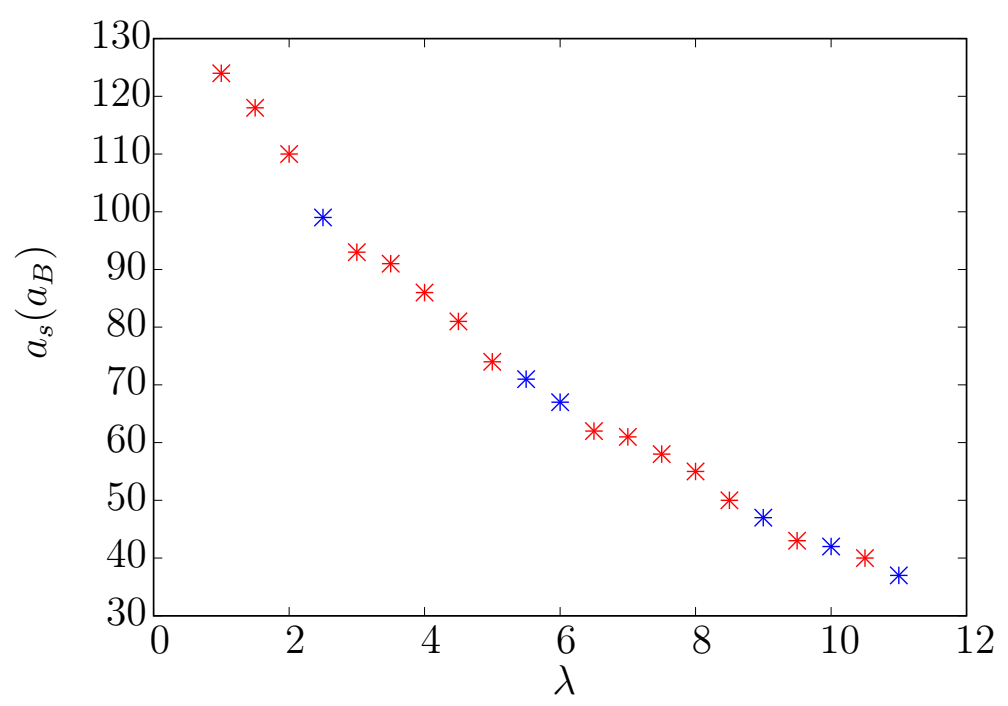

Figure 3.1.: Stability diagram for a BEC of $N=20000$ dysprosium atoms with $\tilde{\omega}=2 \pi \cdot 70 s^{-1}$ (see text). The $x$-axis shows the trap aspect ratio $\lambda=\omega_{z} / \omega_{r}$ characterizing fully the shape of the trap and the $y$-axis is given in units of the Bohr radius $a_{B}$. Red stars denote condensates with maximal density at the trap center and blue stars condensates with a bloodcell structure.

where $\hat{T}$ and $\hat{V}$ represent the kinetic $\left(-\frac{\hbar^{2} \nabla^{2}}{2 M}\right)$ and potential terms $\left(V_{\mathrm{tr}}+g n+\right.$ $\left.\int d^{3} r^{\prime} V_{\mathrm{dd}}\left(\mathbf{r}-\mathbf{r}^{\prime}\right) n\left(\mathbf{r}^{\prime}\right)\right)$, respectively. We use Fourier transforms to calculate the action of the kinetic and potential terms as they reduce to a simple multiplication for the kinetic term in momentum space and for the potential term in position space. The dipole-dipole potential $V_{\mathrm{dd}}(\mathbf{r})$ needs special treatment as it is singular at $\mathbf{r}=0$. We calculate this term in Fourier space using the convolution theorem

$$
\int d^{3} r^{\prime} V_{\mathrm{dd}}\left(\mathbf{r}-\mathbf{r}^{\prime}\right) n\left(\mathbf{r}^{\prime}\right)=\int \frac{d^{3} k}{(2 \pi)^{3}} e^{\mathrm{i} \mathbf{k} \cdot \mathbf{r}} \tilde{V}_{\mathrm{dd}}(\mathbf{k}) \tilde{n}(\mathbf{k})=F^{-1}\left[\tilde{V}_{\mathrm{dd}}(\mathbf{k}) \tilde{n}(\mathbf{k})\right],
$$

where $F^{-1}$ denotes the inverse Fourier transform and $\tilde{V}_{\mathrm{dd}}(\mathbf{k})$ has been evaluated in section 2.1. Moreover we employ a cut-off of the dipolar potential in order to reduce spurious boundary effects [84]. They stem from the fact that we use a discrete Fourier transform and therefore the system is implicitly considered to be periodic which leads to a three-dimensional periodic lattice of condensates with the numerical box as unit cell. Thus the long-ranged nature of the DDI poses a problem when condensates in different unit cells interact with each other. This problem manifests itself in the non-continuous behavior of $\tilde{V}_{\mathrm{dd}}$ at $\mathbf{k}=0$ where the Fourier transformed density attains its maximum. Since the condensate is isolated and has a finite size the solution to this problem is to introduce a cut-off of the dipole-dipole potential, i.e. setting $V_{\mathrm{dd}}(\mathbf{r})$ to zero for $r>R$, where $R$ is the cut-off radius. This procedure does not change the physics of the system as long as $R$ is bigger than the size of the condensate in any direction. The Fourier transformation of the dipolar potential with cut-off reads

$$
\tilde{V}_{\mathrm{dd}}=\frac{C_{\mathrm{dd}}}{4 \pi}\left(3 \cos ^{2} \vartheta-1\right)\left(1+3 \frac{\cos (R k)}{R^{2} k^{2}}-3 \frac{\sin (R k)}{R^{3} k^{3}}\right),
$$


where $k=|\mathbf{k}|$.

We determine the stability threshold as the smallest scattering length value for which we find a stable solution and the results can be seen in Fig. (3.1). The condensate is unstable if the scattering length takes a value below the curve and is stable above. The stability diagram shows regions in which the BEC has a profile in which the maximal density is at the trap center (normal condensates) and BECs, where the maximum of the density lies at a ring around the trap center (bloodcell condensates) [85].

The calculations are valid in general as the system parameters can be translated to other dipolar systems via $N_{1}=\left(\frac{\tilde{\omega}_{\mathrm{D}} M_{\mathrm{D}}^{3}}{\tilde{\omega}_{1} M_{1}^{3}}\right)^{\frac{1}{2}}\left(\frac{\mu_{\mathrm{D}}}{\mu_{1}}\right)^{2} N_{\mathrm{D}}$ and $a_{\mathrm{s}, 1}=\frac{M_{1} \mu_{1}^{2}}{M_{\mathrm{D}} \mu_{\mathrm{D}}^{2}} a_{s, \mathrm{D}}$, where the subscript $\mathrm{D}$ denotes the system parameters we are using for dysprosium and "1" the parameters to which it is desired to transform. Thus Fig. (3.1) describes also the stability diagram for the experimental relevant case of an erbium condensate of $N=40000$ atoms with $\tilde{\omega}=2 \pi \cdot 70 \mathrm{~s}^{-1}$, where the critical scattering length values have to be divided by a factor of roughly 2 .

\subsection{Bogoliubov spectrum}

\subsubsection{Framework}

We study the excitations of the dipolar condensate by calculating the Bogoliubov spectrum numerically for different trapping geometries. As this procedure is elaborate for a three-dimensional dipolar system we give a detailed description of the method we have used. First we introduce $f_{+}=u+v$ and $f_{-}=u-v$ which transforms the Eqs. (2.6) into

$$
\begin{aligned}
E f_{+}(\mathbf{r})= & {\left[-\frac{\hbar^{2} \nabla^{2}}{2 M}+V_{\mathrm{tr}}(\mathbf{r})+g n(\mathbf{r})+\int d^{3} r^{\prime} V_{\mathrm{dd}}\left(\mathbf{r}-\mathbf{r}^{\prime}\right) n\left(\mathbf{r}^{\prime}\right)-\mu\right] f_{-}(\mathbf{r}), } \\
E f_{-}(\mathbf{r})= & {\left[-\frac{\hbar^{2} \nabla^{2}}{2 M}+V_{\mathrm{tr}}(\mathbf{r})+3 g n(\mathbf{r})+\int d^{3} r^{\prime} V_{\mathrm{dd}}\left(\mathbf{r}-\mathbf{r}^{\prime}\right) n\left(\mathbf{r}^{\prime}\right)-\mu\right] f_{+}(\mathbf{r}) } \\
& +2 \Psi(\mathbf{r}) \int d^{3} r^{\prime} V_{\mathrm{dd}}\left(\mathbf{r}-\mathbf{r}^{\prime}\right) \Psi\left(\mathbf{r}^{\prime}\right) f_{+}\left(\mathbf{r}^{\prime}\right)
\end{aligned}
$$

where we have chosen the condensate wave function $\Psi$ to be real. Inserting Eq. (3.6) into Eq. (3.5) yields a single equation for $f_{+}$decoupled from $f_{-}$

$$
\begin{aligned}
E^{2} f_{+}(\mathbf{r})= & {\left[-\frac{\hbar^{2} \nabla^{2}}{2 M}+V_{\mathrm{tr}}(\mathbf{r})+g n(\mathbf{r})+\int d^{3} r^{\prime} V_{\mathrm{dd}}\left(\mathbf{r}-\mathbf{r}^{\prime}\right) n\left(\mathbf{r}^{\prime}\right)-\mu\right] } \\
& \left(\left[-\frac{\hbar^{2} \nabla^{2}}{2 M}+V_{\mathrm{tr}}(\mathbf{r})+3 g n(\mathbf{r})+\int d^{3} r^{\prime} V_{\mathrm{dd}}\left(\mathbf{r}-\mathbf{r}^{\prime}\right) n\left(\mathbf{r}^{\prime}\right)-\mu\right] f_{+}(\mathbf{r})\right. \\
& \left.+2 \Psi(\mathbf{r}) \int d^{3} r^{\prime} V_{\mathrm{dd}}\left(\mathbf{r}-\mathbf{r}^{\prime}\right) \Psi\left(\mathbf{r}^{\prime}\right) f_{+}\left(\mathbf{r}^{\prime}\right)\right)
\end{aligned}
$$

The procedure is then as follows. First we have to calculate a stable ground state solution $\Psi$ via imaginary time evolution of Eq. (3.1). Second we insert this result into Eq. (3.7) and afterwards solve Eq. (3.5) to calculate $f_{-}$.

Solving the Eqs. (3.5) and (3.7) numerically is challenging as this requires solving the eigenvalue and eigenvector problem for the three-dimensional functions 
$f_{+}$and $f_{-}$. Moreover, cylindrical symmetry cannot be used in a simple way as the dipolar terms have to be calculated in Fourier space. In Ref. [84] Hankel transformations were used in order to reduce the dimensionality of the problem. However no Fast-Hankel transforms could be used in a reasonable way so that the gain in speed by using Hankel transforms is small. Therefore, we use a different way in which we project Eqs. (3.5) and (3.7) to a harmonic oscillator basis. The solution of the BdG equations should be described well with a small amount of basis states as long as the interactions are small enough. Thus, we expand $f_{ \pm}(\mathbf{r})=\sum_{k m i} c_{k m i}^{( \pm)} \varphi_{k m}(\rho) \varphi_{m}(\phi) \varphi_{i}(z)$ and solve the resulting the linear equations for the expansion coefficients $c_{k m i}^{ \pm}$with LAPACK routines [135]. The basis functions take the form

$$
\begin{aligned}
\varphi_{k m}(\rho) & =\sqrt{\frac{k !}{\pi(k+|m|) !}}\left(\frac{M \omega_{r}}{\hbar}\right)^{\frac{1}{2}} e^{-\frac{M \omega_{r} \rho^{2}}{2 \hbar}}\left(\sqrt{\left.\frac{M \omega_{r}}{\hbar} \rho\right)^{|m|} L_{k}^{|m|}\left(\frac{M \omega_{r}}{\hbar} \rho^{2}\right)}\right. \\
\varphi_{m}(\phi) & =\mathrm{e}^{\mathrm{i} m \phi} \\
\varphi_{i}(z) & =\frac{1}{\sqrt{2^{i} i !}}\left(\frac{M \omega_{z}}{\pi \hbar}\right)^{\frac{1}{4}} e^{-\frac{M \omega_{z} z^{2}}{2 \hbar}} H_{i}\left(\sqrt{\frac{M \omega_{z}}{\hbar} z}\right)
\end{aligned}
$$

where $L_{k}^{|m|}$ are the generalized Laguerre polynomials and $H_{i}$ the Hermite polynomials. We have chosen a common basis of the Hamiltonian of the non-interacting system and the $z$-component of the angular momentum operator $\hat{L}_{z}$ so that we can use the projection of angular momentum as quantum number for the system. The projection procedure is at follows. Let us write Eq. (3.7) in the coordinatefree form $E^{2}\left|f_{+}\right\rangle=\hat{M}^{+}\left|f_{+}\right\rangle$and multiply it from the left by a basis state $\langle k m i|$ leading to

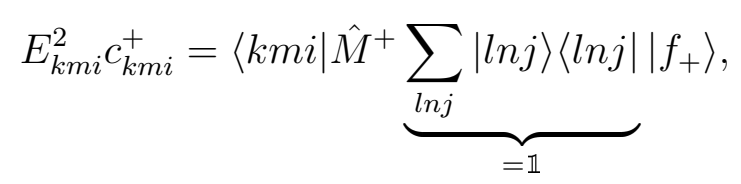

where $\mathbb{1}$ represents the identity. This can be rewritten into the matrix form

$$
E_{k m i}^{2} c_{k m i}^{+}=\sum_{l n j} M_{k m i l n j}^{+} c_{l n j}^{+}
$$

where the matrix elements $M_{k m i l n j}^{+}=\left\langle k m i\left|\hat{M}^{+}\right| \ln j\right\rangle$ have to be calculated via integration over the whole position space. Accordingly, Eq. (3.7) takes the form

$$
E_{k m i} c_{k m i}^{+}=\sum_{l n j} M_{k m i l n j}^{-} c_{l n j}^{-}
$$

with the matrix $M_{k m i l n j}^{-}$. Since states with different $m$ do not mix the full matrix splits into different blocks which can be calculated separately. Therefore the matrix dimension is small and the calculation of the integrals remains as the only challenging task. A detailed discussion of the calculation of the integrals and the concrete forms of matrices $M^{+}$and $M^{-}$can be found in appendix B. 

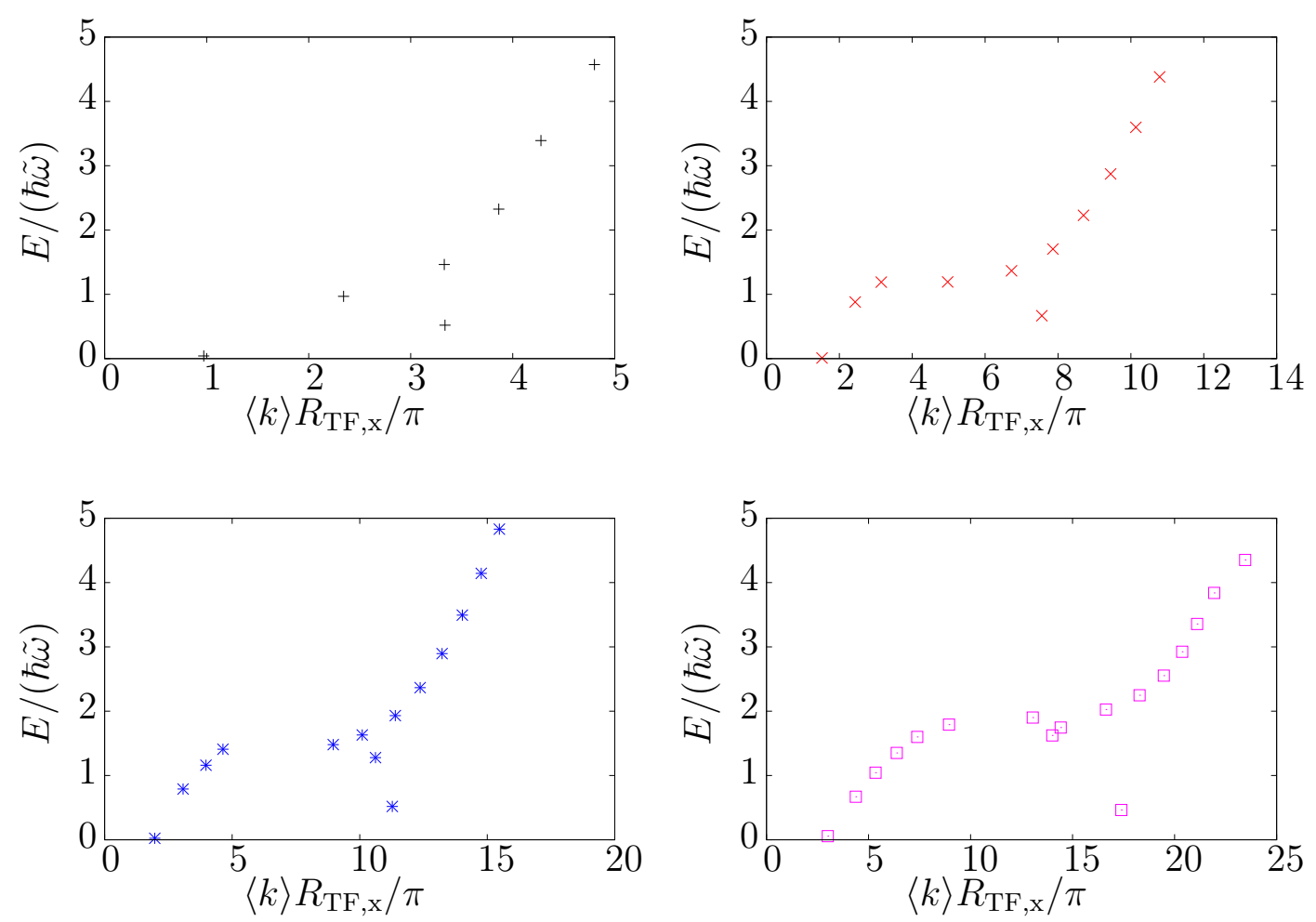

Figure 3.2.: The Bogoliubov spectrum of a trapped dipolar BEC shows a crossover from the phonon-to-roton regime. The calculations are done for condensates $N=20000$ dysprosium atoms with $\tilde{\omega}=70 \cdot 2 \pi s^{-1}$ for different trap aspect ratios (black cross: $\lambda=2$, red saltier $\lambda=6$, blue star: $\lambda=10$, purple square: $\lambda=20$ ) with $m=0$. The linear momentum is rescaled in units of the Thomas-Fermi radius $R_{\mathrm{TF}}$ showing that the most unstable mode becomes increasingly local for larger trap aspect ratios.

\subsubsection{Phonon-to-roton crossover}

We characterize the Bogoliubov excitations obtained from Eqs. (3.5) and (3.7) by two different quantum numbers, their projection of angular momentum $m$ and an effective linear momentum [90] which is given as the expectation value of the in-plane momentum $\langle k\rangle=\sqrt{\left\langle k_{r}^{2}\right\rangle}$ perpendicular to the dipoles. Thus, we apply

$$
\langle k\rangle_{j}=\sqrt{\frac{\int d^{3} k\left(k_{x}^{2}+k_{y}^{2}\right)\left[\left|\hat{u}_{j}(\mathbf{k})\right|^{2}+\left|\hat{v}_{j}(\mathbf{k})\right|^{2}\right]}{\int d^{3} k\left[\left|\hat{u}_{j}(\mathbf{k})\right|^{2}+\left|\hat{v}_{j}(\mathbf{k})\right|^{2}\right]}} .
$$

where time-dependent cross terms proportional to $\cos (2 \omega t)$ have been time averaged. The discrete modes for each $m$ follow a dependence on the effective momentum that resembles the continuous spectrum of an homogeneous system. Results for condensates within traps with $\lambda=2,6,10$, and 20 can be seen in Fig. (3.2). We chose in all of these results a scattering length slightly above the stability threshold at which the contact strength is only about $0.5 \%$ bigger than the critical value. The lowest mode which can be seen is always a mode with energy equal to zero which corresponds to a gauge transformation (see section 1.6). The mode causing the instability is in all figures the mode lying clearly 
under the quasi-continuous curve (for simplicity we will call this mode the mostunstable mode, although in Fig. (3.2) the mode is not yet unstable). Fig. (3.2) shows that the wavelength of the most unstable mode becomes increasingly local, and that the number of states with smaller effective momentum than the most unstable mode increases significantly when the trap aspect ratio grows. For a small trap aspect ratio $(\lambda=2)$ the most unstable mode has a phononic character, having a wavelength comparable to the condensate size. On the contrary for higher trap aspect ratios the most unstable mode presents a very short wavelength compared to the condensate radius, and hence the instability will acquire a roton-like character. There is hence a smooth crossover from phonon-like to roton-like instability.

\subsection{Post-destabilization dynamics}

The dynamics following the condensate destabilization offers an interesting (and experimentally accessible) insight in the nature of the most unstable mode. In order to explore this dynamics, we calculate a stable ground state solution and perform afterwards a real-time evolution with a scattering length decreased below the stability threshold. We employ for the real-time evolution the same techniques as for the imaginary time evolution.
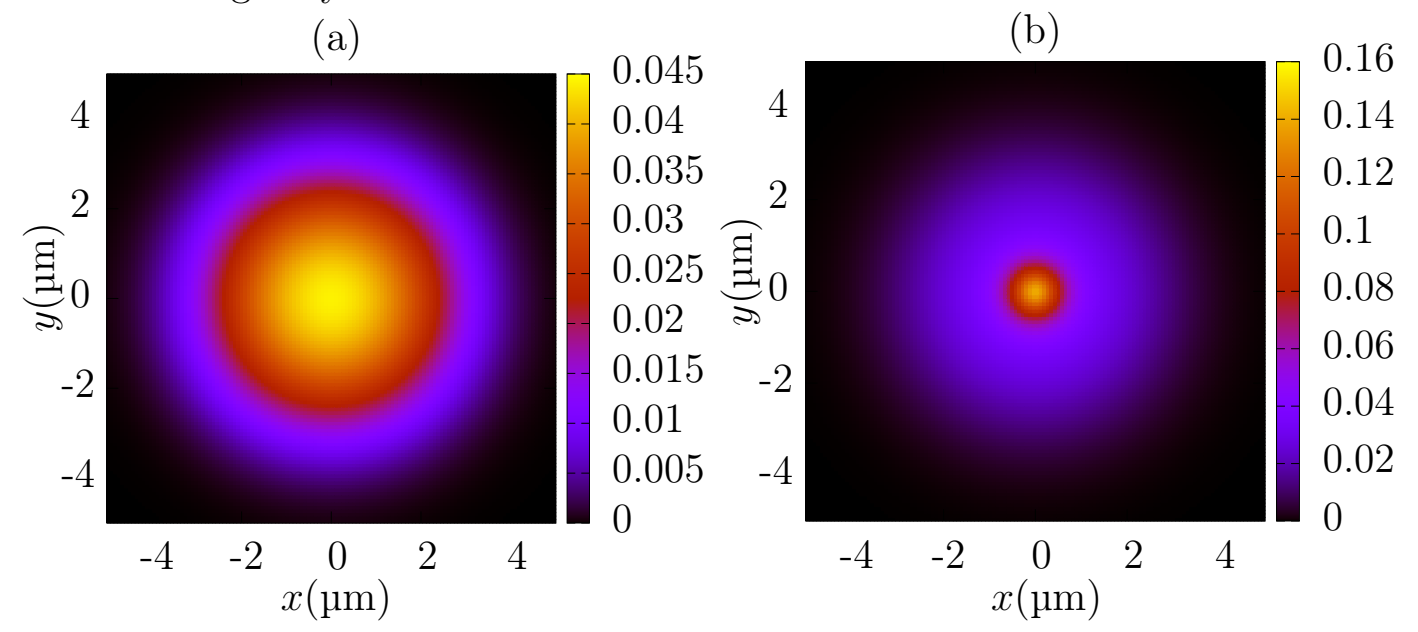

Figure 3.3.: Integrated density profile $n_{X Y}(x, y) / N$, with $n_{X Y}(x, y)=\int d z n(\mathbf{r})$, for a BEC of $N=20000$ dysprosium atoms with $\tilde{\omega}=70 \cdot 2 \pi \mathrm{s}^{-1}$ and $\lambda=2$ initially formed with $a_{s}=110 a_{B}$ (a) and $6.8 \mathrm{~ms}$ after a quench to $a_{s}=106 a_{B}(\mathrm{~b})$. The figure shows a symmetric phononic collapse which affects most of the condensed atoms.

The density fluctuations $\delta n$ caused by the Bogoliubov modes can be calculated according to

$$
\delta n(\mathbf{r})=\Psi(\mathbf{r})\left[f_{+}(\mathbf{r}) \mathrm{e}^{-\mathrm{i} \frac{E}{\hbar} t}+f_{+}^{*}(\mathbf{r}) \mathrm{e}^{\mathrm{i} \frac{E}{\hbar} t}\right],
$$

so that density fluctuations are directly connected to $f_{+}$. Unstable Bogoliubov modes become imaginary and therefore their density fluctuations increase exponentially over time so that the collapse dynamics reveal basic properties of the mode causing the collapse if we cross slightly the instability threshold such that only a single mode becomes unstable. 
Collapse dynamics for dipolar condensates, e.g. chromium atoms, were already theoretically investigated [94], but their locality character was not studied for different trap aspect ratios $\lambda$. A condensate with a bloodcell profile undergoes an angular collapse in which a mode with $m \neq 0$ drives the collapse and several peaks are nucleated at the ring-like high density region around the trap center [94]. In contrast a condensate with normal density profile collapses at the trap center. We investigate both situations for different trap aspect ratios starting with normal condensates, i.e. with no blood-cell structure.

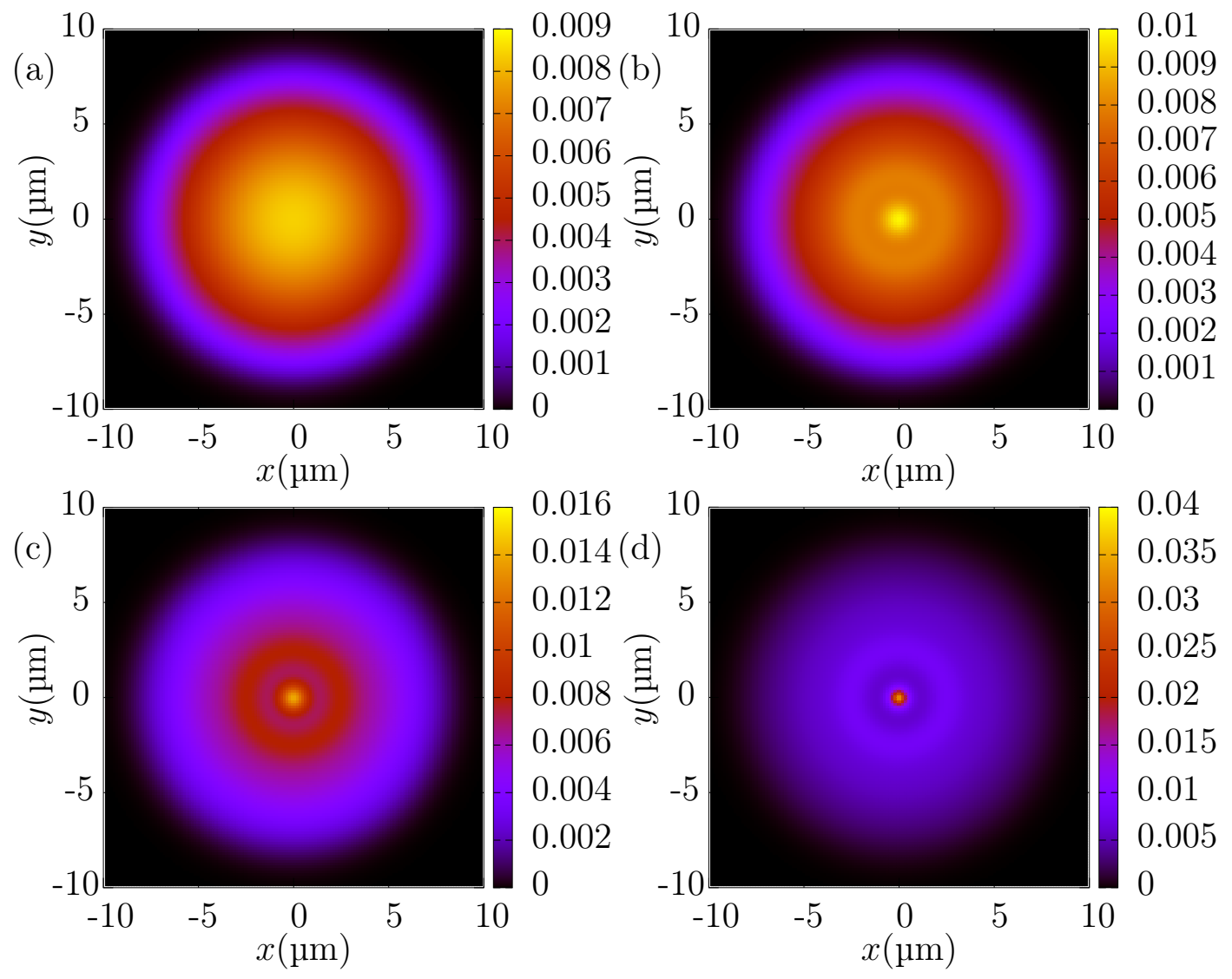

Figure 3.4.: Integrated density profile $n_{X Y}(x, y) / N$ for a BEC of $N=20000$ dysprosium atoms with $\tilde{\omega}=70 \cdot 2 \pi s^{-1}$ and $\lambda=8$ initially formed with $a_{s}=55 a_{B}$ (a). During the collapse the density fluctuations caused by the unstable modes become exponentially increased. Therefore, several rings are formed showing the small wavelength of the most unstable mode ((b), $11.4 \mathrm{~ms}$ after a quench to $52 a_{B}$ and (c) $13.6 \mathrm{~ms}$ after the quench). Finally the condensate collapses at the trap center ((d), $14.8 \mathrm{~ms}$ after the quench). The figure shows the more local character of the most unstable mode in the condensate with higher trap aspect ratio.

We study the integrated density profiles $n_{\mathrm{XY}}(x, y)=\int d z n(\mathbf{r}) / N$ for different times after the quench of the scattering length driving the instability. The results for $\lambda=2$ can be seen in Fig. 3.3. The density profile changes very slowly during the first $4 \mathrm{~ms}$ of the collapse. Then the collapse dynamics becomes faster and the central region with a radius of $\sim 50 \%$ of the condensate radius collapses within the next $4 \mathrm{~ms}$ as can be seen in Fig. 3.3b. 
(a)

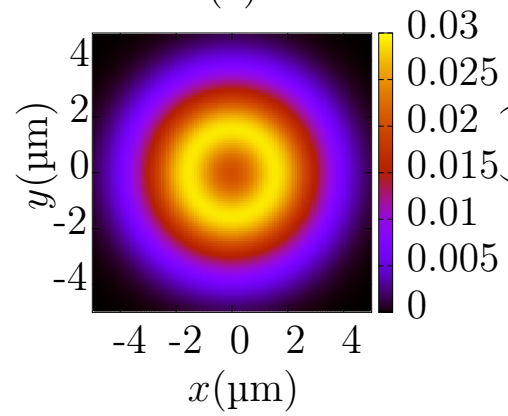

(d)

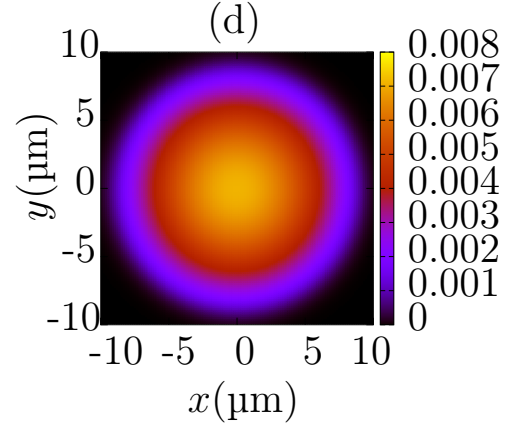

(b)

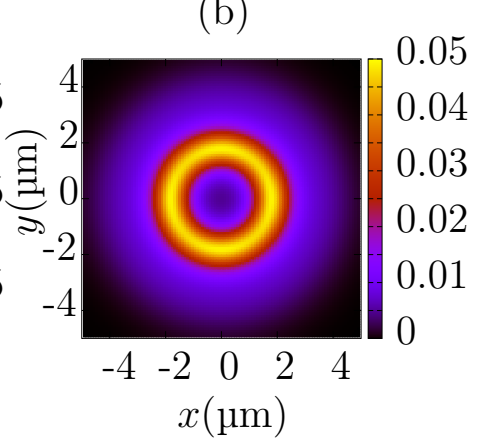

(e)

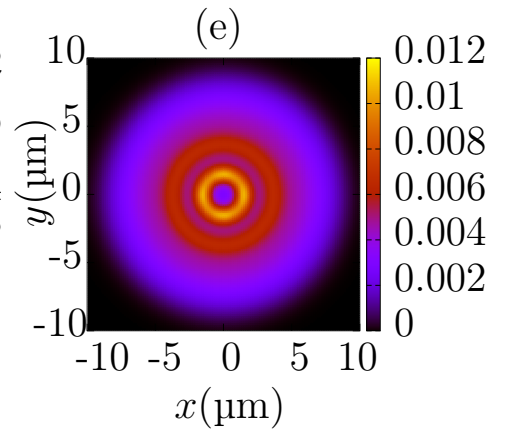

(c)

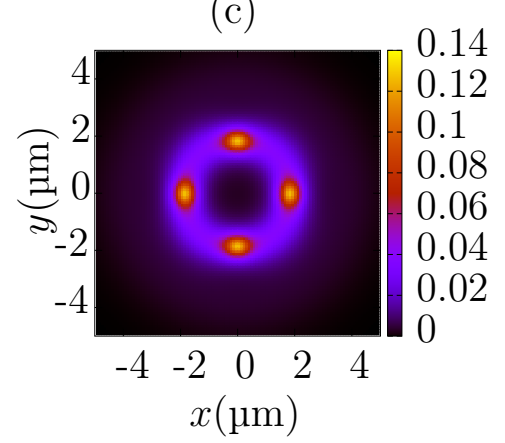

(f)

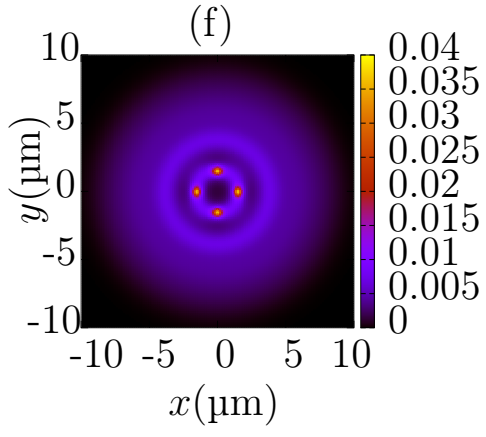

Figure 3.5.: Integrated density profile $n_{X Y}(x, y) / N$ for a BEC of $N=20000$ dysprosium atoms with $\tilde{\omega}=70 \cdot 2 \pi s^{-1}$ and $\lambda=2.5(\mathrm{a}-\mathrm{c})$ and $\lambda=$ 10 (d-f). The condensate for $\lambda=2.5$ is initially formed with $a_{s}=$ $100 a_{B}$ (a) and has a pronounced bloodcell structure. During the collapse dynamics the density of the ring-like structure increases (b, $6.81 \mathrm{~ms}$ after a quench to $a_{s}=96 a_{B}$ ) and finally four peaks are nucleated which collapse individually (c, $9.5 \mathrm{~ms}$ after the quench). The condensate for $\lambda=10$ is initially formed with $a_{s}=42 a_{B}$ with only a slightly bloodcell character (d). During the collapse dynamics the density of the ring-like structure increases and several additional rings form (e, $13 \mathrm{~ms}$ after a quench to $a_{s}=39 a_{B}$ ). At the end several peaks are nucleated on the initial ring at the trap center which collapse as in the case for $\lambda=2$ (f, $14.8 \mathrm{~ms}$ after the quench).

In contrast the collapse dynamics for a normal condensate with $\lambda=8$ can be seen in Fig. 3.4. The dynamics is again slow at the beginning. The central density increases in a region with a radius of roughly $10 \%$ of the Thomas Fermi radius and additional ring like structures become visible after $8 \mathrm{~ms}$ showing the small wavelength of the mode driving the collapse. The outer regions are almost unaffected by the dynamics in the inner part of the condensate. Therefore, the most unstable mode has a much more local character than in the case of $\lambda=2$.

Condensates with a bloodcell profile collapse in a different way. Their initial ring structures become more pronounced during the collapse as can be seen in Fig. (3.5a, b, d, e) until peaks are nucleated on the ring which grow in time. This breaks the cylindrical symmetry of the BEC, which collapses then at several different points $(3.5 \mathrm{c}, \mathrm{f})$. The symmetry breaking is a direct consequence of the fact that the mode which causes the collapse has a non-zero projection of angular momentum $m \neq 0$ and is therefore not cylindrically symmetric itself. For $\lambda=2.5$ the radius of the initial ring is comparable to the radius of the whole condensate, where in the case of $\lambda=10$ the ring structure is very local and additional rings 
form in the outer regions of the condensate. This shows clearly the roton-like character of the mode driving the instability for $\lambda=10$.

\subsection{Conclusions}

In summary we have determined the stability boundary for condensates consisting of highly magnetic atoms, i.e. dysprosium and erbium, and have shown a crossover from a phonon to roton unstable condensate, in which the wavelength of the most unstable mode decrease with respect to the size of the condensate in the plane perpendicular to the dipole direction.

As can be seen in the next chapters quantum fluctuations play an important role in strongly dipolar systems close to instability where they can even stabilize the condensate. However, the techniques employed for the study of quantum fluctuations which will be introduced in the next chapter cannot be used to investigate condensates with scattering lengths values much smaller than the stability threshold of a homogeneous dipolar BEC, or trapped aspect ratios larger than $\lambda=3$. Therefore, it is yet unclear in which way the results of this chapter, and especially the post-quenching dynamics, are altered due to quantum fluctuations. 


\section{Higher order corrections}

In the previous chapters we concentrated on the properties of the condensate itself, thereby neglecting non-condensed particles and their effects. However, some particles remain uncondensed due to interactions and non-zero temperature. This chapter is devoted to present explicit expressions in order to take the effects of these non-condensed particles into account. We discuss these corrections for a homogeneous contact interacting BEC in section 4.1 followed by a description of a multicomponent system in section 4.2 which consists of a Bose-Bose mixture in which it can be shown that the higher order corrections can become significant for the stability of BECs. Section 4.3 is dedicated to dipolar systems and in section 4.4 we discuss the extension to non-homogeneous systems.

\subsection{Lee-Huang-Yang corrections}

Consider a homogeneous BEC interacting solely via short-ranged contact interactions. As the system is translationally invariant we perform the calculations in momentum space. This discussion can be found for instance in Ref. [17]. The field operator can be expanded in terms of the eigenstates of the momentum operator

$$
\hat{\Psi}(\mathbf{r})=\sum_{\mathbf{p}} \hat{a}_{\mathbf{p}} \frac{1}{\sqrt{V}} e^{\mathrm{i} \frac{\mathbf{p} \cdot \mathbf{r}}{\hbar}},
$$

where $\hat{a}_{\mathbf{p}}$ is the annihilation operator of a particle with momentum $\mathbf{p}$ satisfying Bose commutation relations $\left[\hat{a}_{\mathbf{p}}, \hat{a}_{\mathbf{p}^{\prime}}\right]=0,\left[\hat{a}_{\mathbf{p}}^{\dagger}, \hat{a}_{\mathbf{p}^{\prime}}^{\dagger}\right]=0$ and $\left[\hat{a}_{\mathbf{p}}, \hat{a}_{\mathbf{p}^{\prime}}^{\dagger}\right]=\delta_{\mathbf{p p}^{\prime}}$. Inserting Eq. (4.1) into the definition of the Hamiltonian Eq. (1.8) yields

$$
\hat{H}=\sum_{\mathbf{p}} \frac{\mathbf{p}^{2}}{2 M} \hat{a}_{\mathbf{p}}^{\dagger} \hat{a}_{\mathbf{p}}+\frac{1}{2 V} \sum_{\mathbf{p}_{1} \mathbf{p}_{2} \mathbf{p}} \tilde{V}_{\text {int }}(\mathbf{p}) \hat{a}_{\mathbf{p}_{1}+\mathbf{p}}^{\dagger} \hat{a}_{\mathbf{p}_{2}-\mathbf{p}}^{\dagger} \hat{a}_{\mathbf{p}_{1}} \hat{a}_{\mathbf{p}_{2}},
$$

where $\tilde{V}_{\text {int }}(\mathbf{p})=\tilde{V}_{\text {int }}(0)$ for a contact interaction. Assuming a condensate in $\mathbf{p}=0$, we may approximate $\hat{a}_{0}, \hat{a}_{0}^{\dagger} \approx \sqrt{N_{0}}$ and then expand the Hamiltonian up to quadratic terms in the creation and annihilation operator which leads to

$$
\hat{H}=\sum_{\mathbf{p}} \frac{\mathbf{p}^{2}}{2 M} \hat{a}_{\mathbf{p}}^{\dagger} \hat{a}_{\mathbf{p}}+\frac{N_{0}^{2}}{2 V} \tilde{V}_{\mathrm{int}}(0)+\frac{N_{0}}{2 V} \tilde{V}_{\mathrm{int}}(0) \sum_{\mathbf{p}}\left[4 \hat{a}_{\mathbf{p}}^{\dagger} \hat{a}_{\mathbf{p}}+\hat{a}_{\mathbf{p}}^{\dagger} \hat{a}_{-\mathbf{p}}^{\dagger}+\hat{a}_{\mathbf{p}} \hat{a}_{-\mathbf{p}}\right] .
$$

Note that $N_{0}=N-\sum_{\mathbf{p}} \hat{a}_{\mathbf{p}}^{\dagger} \hat{a}_{\mathbf{p}}$, and hence $N_{0}^{2} \approx N^{2}-2 N \sum_{\mathbf{p}} \hat{a}_{\mathbf{p}}^{\dagger} \hat{a}_{\mathbf{p}}$ which yields

$$
\hat{H}=\sum_{\mathbf{p}} \frac{\mathbf{p}^{2}}{2 M} \hat{a}_{\mathbf{p}}^{\dagger} \hat{a}_{\mathbf{p}}+\frac{N^{2}}{2 V} \tilde{V}_{\mathrm{int}}(0)+\frac{N}{2 V} \tilde{V}_{\mathrm{int}}(0) \sum_{\mathbf{p}}\left[2 \hat{a}_{\mathbf{p}}^{\dagger} \hat{a}_{\mathbf{p}}+\hat{a}_{\mathbf{p}}^{\dagger} \hat{a}_{-\mathbf{p}}^{\dagger}+\hat{a}_{\mathbf{p}} \hat{a}_{-\mathbf{p}}\right] .
$$

$\tilde{V}_{\text {int }}(0)$ must be expressed in terms of the scattering length $a_{s}$. In the second order terms we may use $\tilde{V}_{\text {int }}(0)=\frac{4 \pi \hbar^{2} a_{s}}{M}=g$, but in the term $\frac{N^{2}}{2 V} \tilde{V}_{\text {int }}(0)$ we 


\section{Higher order corrections}

need to go to second order in the Born approximation which reads $\tilde{V}_{\text {int }}(0)=$ $g\left(1+\frac{g}{V} \sum_{\mathbf{p}} \frac{M}{\mathbf{p}^{2}}\right)[17]$. Taking this into account yields

$$
\hat{H}=\sum_{\mathbf{p}} \frac{\mathbf{p}^{2}}{2 M} \hat{a}_{\mathbf{p}}^{\dagger} \hat{a}_{\mathbf{p}}+\frac{g N^{2}}{2 V}+\frac{g^{2} N^{2}}{2 V^{2}} \sum_{\mathbf{p} \neq 0} \frac{M}{p^{2}}+\frac{g N}{2 V} \sum_{\mathbf{p} \neq 0}\left(2 \hat{a}_{\mathbf{p}}^{\dagger} \hat{a}_{\mathbf{p}}+\hat{a}_{\mathbf{p}} \hat{a}_{-\mathbf{p}}+\hat{a}_{\mathbf{p}}^{\dagger} \hat{a}_{-\mathbf{p}}^{\dagger}\right) .
$$

The Hamiltonian (4.5) is quadratic in the operators $\hat{a}_{\mathbf{p}}$ and $\hat{a}_{\mathbf{p}}^{\dagger}$ and can be diagonalized by means of the Bogoliubov transformation $\hat{a}_{\mathbf{p}}=u_{\mathbf{p}} \hat{b}_{\mathbf{p}}+v_{-\mathbf{p}}^{*} \hat{b}_{-\mathbf{p}}^{\dagger}$ which introduces a new set of operators $\hat{b}_{\mathbf{p}}$. We impose for them the usual Bose commutation relations which is ensured by demanding the condition $\left|u_{\mathbf{p}}\right|^{2}-\left|v_{-\mathbf{p}}\right|^{2}=1$. The Bogoliubov amplitudes $u_{\mathbf{p}}$ and $v_{\mathbf{p}}$ are then chosen in order to make the coefficients of the non-diagonal terms $\hat{b}_{\mathbf{p}}^{\dagger} \hat{b}_{-\mathbf{p}}^{\dagger}$ and $\hat{b}_{\mathbf{p}} \hat{b}_{-\mathbf{p}}$ vanish, which directly yields

$$
\begin{aligned}
& u_{\mathbf{p}}= \pm\left[\frac{\frac{\mathbf{p}^{2}}{2 M}+g n}{2 \epsilon(\mathbf{p})}+\frac{1}{2}\right]^{1 / 2} \\
& v_{\mathbf{p}}=\mp\left[\frac{\frac{\mathbf{p}^{2}}{2 M}+g n}{2 \epsilon(\mathbf{p})}-\frac{1}{2}\right]^{1 / 2},
\end{aligned}
$$

with $\epsilon(\mathbf{p})=\sqrt{\frac{\mathbf{p}^{2}}{2 M}\left(\frac{\mathbf{p}^{2}}{2 M}+2 g n\right)}$ being the Bogoliubov dispersion relation. The condensate depletion at zero temperature can be calculated explicitly by inserting Eqs. (4.6), (4.7) for the Bogoliubov amplitudes and substituting the sum in the equation for the total number of particles by an integral as $\sum_{\mathbf{p}} \rightarrow \frac{V}{(2 \pi \hbar)^{3}} \int d^{3} p$ with the result

$$
\frac{N-N_{0}}{N}=\frac{8}{3 \sqrt{\pi}} \sqrt{n a_{s}^{3}}
$$

Thus, the interactions cause some particles to be pushed out of the condensate so that even at $T=0$ not all particles are condensed into the zero momentum state $^{1}$. The diagonalized Hamiltonian reads

$$
\hat{H}=E_{0}+\sum_{\mathbf{p}} \epsilon(\mathbf{p}) \hat{b}_{\mathbf{p}}^{\dagger} \hat{b}_{\mathbf{p}}
$$

describing a system of non-interacting quasiparticles whose creation and annihilation operators are given by $\hat{b}_{\mathbf{p}}^{\dagger}, \hat{b}_{\mathbf{p}}$. The energy of the quasiparticles follows the Bogoliubov dispersion relation and the ground state of the many-body Hamiltonian corresponds to a vacuum of quasi-particles $\hat{b}_{\mathbf{p}}|0\rangle=0$. The ground state energy of the system differs from the mean-field result and is given by

$$
E_{0}=\frac{g N^{2}}{2 V}+\frac{1}{2} \sum_{\mathbf{p} \neq 0}\left(\epsilon(\mathbf{p})-\frac{\mathbf{p}^{2}}{2 M}-g n+\frac{M g^{2} n^{2}}{\mathbf{p}^{2}}\right)
$$

where the first term coincides with the mean-field energy. The second term represents an energy shift originating solely from quantum fluctuations of the noncondensed particles. Replacing the sum by an integral leads to explicit expressions

\footnotetext{
${ }^{1}$ The finite temperature of a gas leads also to a population of higher momentum states. We do not consider temperature effects in this thesis and, therefore, neglect these contributions.
} 
for the ground state energy and the chemical potential $\mu=\frac{\partial E_{0}}{\partial N}$, which read

$$
\begin{aligned}
E_{0} & =\frac{g N^{2}}{2 V}\left(1+\frac{128}{15 \sqrt{\pi}} \sqrt{n a_{s}^{3}}\right), \\
\mu & =g n\left(1+\frac{32}{3 \sqrt{\pi}} \sqrt{n a_{s}^{3}}\right) .
\end{aligned}
$$

These corrections were first calculated in 1957 by Lee, Huang and Yang [136,137] and are therefore called Lee-Huang-Yang (LHY) corrections. Measurements in the group of C. Salomon in 2010 found a good agreement with the theoretical calculations of these beyond mean-field effects [138,139]. Higher order corrections in the diluteness parameter $n a_{s}^{3}$ are discussed in Refs. [140-142], with the next term proportional to $n a_{s}^{3} \ln \left(n a_{s}^{3}\right)$.

\subsection{Lee-Huang-Yang corrections in binary Bose mixtures}

In the previous sections we outlined the derivation of the LHY corrections for homogeneous condensates with contact interactions. Crucially, the diluteness parameter has to remain small $n a_{s}^{3} \ll 1$ with the consequence that the LHY term is much smaller than the mean-field term, as can be directly seen from Eq. (4.12). Therefore, the Lee-Huang-Yang corrections lead in most systems only to small changes in the static and dynamic properties. However, D. S. Petrov proposed in 2015 a scheme in which the LHY term becomes crucial for the stability of a system and governs most of its static and dynamic properties [143]. We highlight his idea in this section.

Consider a Bose gas composed of two different types of particles labeled as 1 and 2. The individual particles interact solely via a short-ranged contact interaction and are not trapped. Then, the Hamiltonian of this system takes the form

$$
\hat{H}=\int d^{3} r\left(\sum_{i=1,2} \frac{\hbar^{2}\left|\nabla_{\mathbf{r}} \Psi_{i}\left(\mathbf{r}^{\prime}\right)\right|^{2}}{2 M_{i}}+\sum_{i, j=1,2} \frac{g_{i j}}{2} n_{i}(\mathbf{r}) n_{j}(\mathbf{r})\right),
$$

where $\Psi_{i}$ and $n_{i}=\left|\Psi_{i}\right|^{2}$ are the condensate wave function and the density of particles of type $i$. The intraspecies coupling constants are given by $g_{11}$ and $g_{22}$, $g_{12}=g_{21}$ characterizes the interspecies coupling constant and the mass of the individual particles is given by $M_{i}$. The stability of the mixture depends on the inter- and intraspecies coupling constants, where the system is stable if $g_{i i}>0$ and $g_{12}^{2}<g_{11} g_{22}$. The Bogoliubov excitation spectrum for the Bose-Bose mixture yields

$$
E_{\mathbf{k}, \pm}=\hbar \sqrt{\frac{\omega_{1}^{2}+\omega_{2}^{2}}{2} \pm \sqrt{\frac{\left(\omega_{1}^{2}-\omega_{2}^{2}\right)^{2}}{4}+\frac{g_{12}^{2} n_{1} n_{2} k^{4}}{M_{1} M_{2}}}}
$$

where $\omega_{i}=\sqrt{\frac{k^{2}}{2 M_{i}}\left(\frac{k^{2}}{2 M_{i}}+\frac{2 g_{i i} n_{i}}{\hbar^{2}}\right)}$ are the Bogoliubov dispersion relations for the individual components. The LHY corrections for this homogeneous system can 


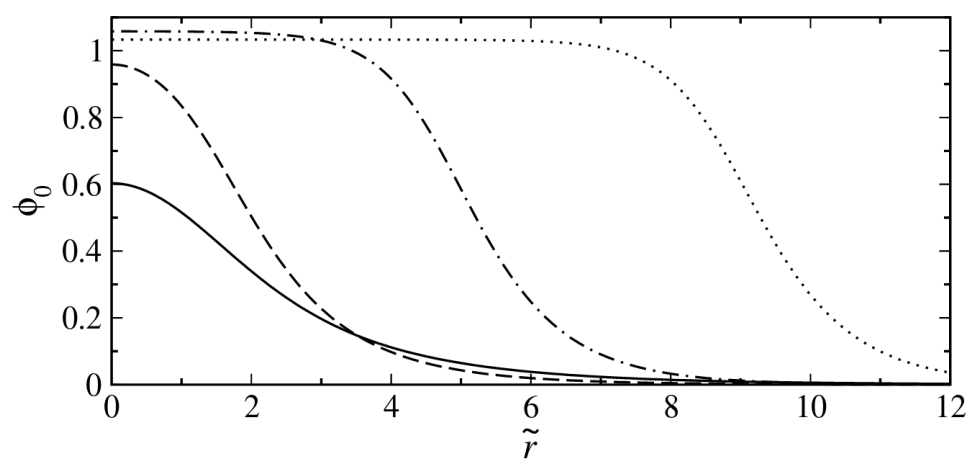

Figure 4.1.: Spatial dependence of the wave function $\phi_{0}$ of a droplet stabilized by the LHY corrections for different particle numbers. A higher particle number leads to a broader wave function. The radial coordinate $\tilde{r}=r / \xi$ was rescaled with respect to $\xi=\sqrt{\frac{3}{2} \frac{\sqrt{g_{22}} / M_{1}+\sqrt{g_{11}} / M_{2}}{|\delta g| \sqrt{g_{11}} n_{1}^{(0)}}}$, where $n_{1}^{(0)}$ is the peak density of the particles of type 1 . The picture was reprinted from [143].

be calculated following a similar procedure as in section 4.1:

$$
\begin{aligned}
\frac{E_{\mathrm{LHY}}}{V}= & \int \frac{d^{3} k}{2(2 \pi)^{3}}\left[E_{\mathbf{k},+}+E_{\mathbf{k},-}-\frac{\hbar^{2} \mathbf{k}^{2}}{2 M_{r}}-g_{11} n_{1}-g_{22} n_{2}\right. \\
& \left.+\frac{M_{1} g_{11}^{2} n_{1}^{2}+M_{2} g_{22}^{2} n_{2}^{2}+4 M_{r} g_{12}^{2} n_{1} n_{2}}{\hbar^{2} \mathbf{k}^{2}}\right] .
\end{aligned}
$$

Note that the excitation spectrum $E_{\mathbf{k}, \pm}$ is always explicitly included in the LHY energy integral.

Let us discuss the situation in the which the parameter $\delta g=g_{12}+\sqrt{g_{11}+g_{22}}$ is negative and much smaller than both $g_{11}$ and $g_{22}$. In this situation the three individual mean-field terms cancel each other almost completely with only a small residual and attractive part remaining leading to a mean-field unstable system. The instability manifests itself in the fact that $E_{\mathbf{k},-}$ becomes complex for small momenta (long-wavelength) $k \sim \sqrt{M|\delta g| n}$. In order to understand what then happens we diagonalize the mean-field term $\sum_{i, j=1,2} g_{i j} \frac{n_{i} n_{j}}{2}$ under the condition that $|\delta g| \ll g_{i i}$ with the result $\lambda_{+} \approx \frac{g_{11}+g_{22}}{2}$ and $\lambda_{-} \approx \frac{\sqrt{g_{11}+g_{22}}}{g_{11}+g_{22}} \delta g$. As $\lambda_{-}$is energetically favorable the density $n_{-}=n_{1} \sqrt{g_{22}}+n_{2} \sqrt{g_{11}}$ increases while the ratio $n_{2} / n_{1}$ is conserved. Hence the attractive mean-field terms try to collapse the mixture.

Let us discuss at this point the effect of the LHY correction. The main contribution of the LHY term stems from hard modes with angular momenta $k \sim \sqrt{M\left|g_{i i}\right| n}$ which are much larger than $\sqrt{M|\delta g| n}$ so that the imaginary part of Eq. (4.15) is orders of magnitude smaller than its real part. Therefore we can safely neglect the contribution of the soft modes in the LHY term and the real part of Eq. (4.15) correctly describes the LHY corrections. As the density increases during a collapse, the mean-field energy grows as $n^{2}$, whereas the energy shift to the ground state stemming from the LHY corrections increases with $n^{5 / 2}$. Hence the LHY term becomes significant for higher densities and eventually restabilizes the system at a critical density. Therefore, the instability caused 
by long-wavelength excitations is balanced by quantum fluctuations with shortwavelength. This leads to the formation of quantum droplets stabilized by a competition of the mean-field terms and the LHY corrections. The profile of several droplet wave functions can be seen in Fig. (4.1).

This stabilization mechanism via quantum fluctuations requires crucially that the system becomes dynamically unstable when the mean-field terms cancel each other as the LHY term can not compete with the individual mean-field terms, but only with the residual mean-field term. Therefore at least two different and competing mean-field terms are necessary.

\subsection{LHY corrections in uniform dipolar BECs}

In 2011 Lima et al. extended the calculation of the LHY corrections to dipolar systems $[144,145]$. The calculation is straightforward as only the interaction term in the Hamilton operator needs to be expanded by an additional dipolar term of the form $\hat{H}_{\mathrm{dd}}=\frac{1}{2} \int d^{3} r d^{3} r^{\prime} \hat{\Psi}^{\dagger}(\mathbf{r}) \hat{\Psi}^{\dagger}\left(\mathbf{r}^{\prime}\right) V_{\mathrm{dd}}\left(\mathbf{r}-\mathbf{r}^{\prime}\right) \hat{\Psi}\left(\mathbf{r}^{\prime}\right) \hat{\Psi}(\mathbf{r})$. Then, the second order Born approximation for the interaction potential is given by

$$
\tilde{V}_{\text {int }}(0)=g+M \int \frac{d^{3} p}{(2 \pi)^{3}} \frac{\tilde{V}_{\text {int }}(-\mathbf{p}) \tilde{V}_{\text {int }}(\mathbf{p})}{\mathbf{p}^{2}},
$$

where $\tilde{V}_{\text {int }}$ contains now both the contact and the dipolar potential (recall from Sec. 2.1 that the dipolar interaction is momentum dependent). Expanding the Hamiltonian up to second order and using Eq. (4.16) leads to the following equations for the quantum depletion and the chemical potential $[144,145]$

$$
\begin{aligned}
\frac{N-N_{0}}{N} & =\frac{8}{3 \sqrt{\pi}} \sqrt{n a_{s}^{3}} G\left(\epsilon_{\mathrm{dd}}\right), \\
\mu & =g n\left[1+\epsilon_{\mathrm{dd}}\left(3 \cos ^{2} \theta-1\right)\right]+\frac{32}{3 \sqrt{\pi}} g n \sqrt{n a_{s}^{3}} F\left(\epsilon_{\mathrm{dd}}\right) .
\end{aligned}
$$

The functions $F\left(\epsilon_{\mathrm{dd}}\right)=\frac{1}{2} \int_{0}^{\pi} d \theta \sin \theta f\left(\epsilon_{\mathrm{dd}}, \theta\right)^{\frac{5}{2}}$ and $G\left(\epsilon_{\mathrm{dd}}\right)=\frac{1}{2} \int_{0}^{\pi} d \theta \sin \theta f\left(\epsilon_{\mathrm{dd}}, \theta\right)^{\frac{3}{2}}$ characterize the dipolar shifts to the chemical potential and the condensate depletion, respectively, and $f\left(\epsilon_{\mathrm{dd}}, \theta\right)=1+\epsilon_{\mathrm{dd}}\left(3 \cos ^{2} \theta-1\right)$. Both functions $F$ and $G$ are monotonous and increase with increasing $\epsilon_{\mathrm{dd}}$ (see Fig. (4.2)) and become imaginary for $\epsilon_{\mathrm{dd}}>1$, which signals a dynamical instability due to the attractive part of the DDI dominating the interactions. Close to instability $\epsilon_{\mathrm{dd}} \sim 1$ the change in the condensate depletion is around $30 \%$ with respect to the non-dipolar case whereas the change in the chemical potential is more pronounced with an increase of around $160 \%$. Surprisingly, albeit the mean-field terms are due to the DDI anisotropic the LHY corrections are isotropic.

\subsection{LHY correction in non-uniform dipolar BECs}

It is hard to derive explicit expressions for the case of inhomogeneous systems. However, if the spatial variations of the condensate density are sufficiently slow the homogeneous results should correctly describe the local behavior of the inhomogeneous gas. This approach is called the local density approximation (LDA). 


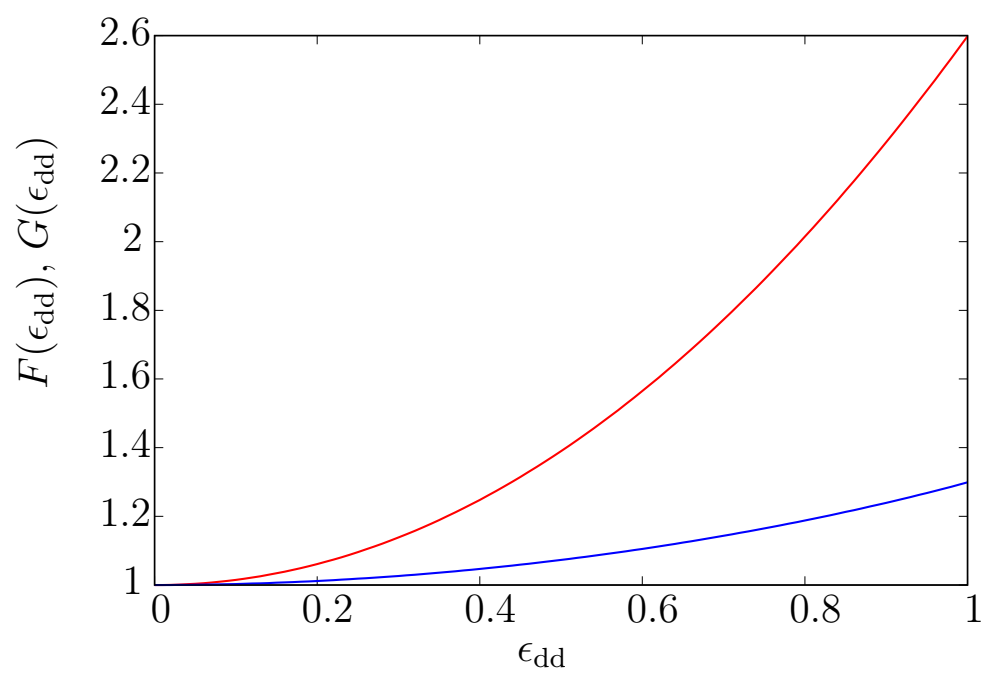

Figure 4.2.: The Functions $F\left(\epsilon_{\mathrm{dd}}\right)$ (red curve) and $G\left(\epsilon_{\mathrm{dd}}\right)$ (blue curve) which govern the dipolar shifts to the ground state energy and condensate depletion plotted in the mean-field stable regime $0 \leq \epsilon_{\mathrm{dd}} \leq 1$.

A rigorous derivation was given in 1997 by K. Huang et al. who considered a spatial dependent Bogoliubov transformation of the form [146]

$$
\delta \hat{\psi}(\mathbf{r})=\int d^{3} r^{\prime}\left[X\left(\mathbf{r}, \mathbf{r}^{\prime}\right) \hat{\xi}\left(\mathbf{r}^{\prime}\right)-Y\left(\mathbf{r}, \mathbf{r}^{\prime}\right) \hat{\xi}^{\dagger}\left(\mathbf{r}^{\prime}\right)\right]
$$

which generalizes the Bogoliubov transformation for homogeneous systems and $\delta \hat{\psi}$ is defined in Eq. (1.10). As expected this transformation is in general nonlocal and introduces a new set of creation and annihilation operators $\hat{\xi}, \hat{\xi}^{\dagger}$. As we consider systems whose spatial variations are sufficiently slow it is helpful to switch to the Wigner representation [147], which is defined as Fourier transforming a function $f\left(\mathbf{x}, \mathbf{x}^{\prime}\right)$ with respect to its relative coordinate $\mathbf{r}=\mathbf{x}-\mathbf{x}^{\prime}$

$$
f_{\mathrm{W}}(\mathbf{R}, \mathbf{p})=\int d^{3} r f\left(\mathbf{R}+\frac{\mathbf{r}}{2}, \mathbf{R}-\frac{\mathbf{r}}{2}\right) e^{\mathrm{i} \cdot \mathbf{r}}
$$

Then integrals of the from $C\left(\mathbf{x}, \mathbf{x}^{\prime}\right)=\int d^{3} z A(\mathbf{x}, \mathbf{z}) B\left(\mathbf{z}, \mathbf{x}^{\prime}\right)$ can be written in terms of a systematic gradient expansion in Wigner representation as

$$
C_{\mathrm{W}}(\mathbf{R}, \mathbf{p})=A_{\mathrm{W}}(\mathbf{R}, \mathbf{p}) B_{\mathrm{W}}(\mathbf{R}, \mathbf{p})+\frac{1}{2 \mathrm{i}} \sum_{j=1}^{3}\left[\frac{\partial A_{\mathrm{W}}}{\partial R_{j}} \frac{\partial B_{\mathrm{W}}}{\partial p_{j}}-\frac{\partial A_{\mathrm{W}}}{\partial p_{j}} \frac{\partial B_{\mathrm{W}}}{\partial R_{j}}\right]+\cdots
$$

The higher oder terms contain the spatial and momentum derivatives of the underlying functions. Neglecting all terms except the first one leads again to local equations from which the LHY corrections can be calculated in the same way as in the homogeneous case [146]. Therefore, the LHY corrections for nonhomogeneous systems using the LDA read [144-146]

$$
\Delta \mu_{\mathrm{LHY}}(\mathbf{r})=\frac{32}{3 \sqrt{\pi}} g n(\mathbf{r}) \sqrt{n(\mathbf{r}) a_{s}^{3}} F\left(\epsilon_{\mathrm{dd}}\right) .
$$


All the individual terms are now position dependent and contain the external trapping potential. This expression is valid when the main contribution of the LHY corrections stems from modes whose momenta are much larger than the typical size of the condensate (see appendix A). Then, as the wavelengths of these momenta are much smaller than the condensate size, the spatial variations of the condensate density are small over one wavelength of these modes validating a description of a locally homogeneous system. This condition is always satisfied in the Thomas-Fermi regime as explained in Refs. [144, 145]. 


\section{Quantum filaments}

In 2015 T. Pfau's group performed highly influential experiments in ultracold dysprosium gases [76]. They revealed the surprising result that the dipole-driven collapse was arrested at high densities and long-lived quantum filaments were formed in a timescale of a few ms. Mean-field models cannot account for this phenomenon, showing that a novel previously neglected stabilization mechanism was at work. As the droplets are stabilized at higher densities the stabilization mechanism seems to depend on density and the first attempt for an explanation were large conservative three-body interactions. This results in the formation of stable droplets if the conservative part of the three-body interaction is two to three orders of magnitude larger than the non-conservative contribution $[148,149]$. However, no conclusive explanation for these large values could be given.

In this chapter we show that the dipole-driven collapse of a dipolar BEC can be arrested by quantum fluctuations. As these are always present in a BEC we do not propose a new interaction in the system, but reveal a situation in which the small effect of quantum fluctuations can become crucial for the static and dynamic properties of the dipolar BEC. In fact the dipole-driven collapse which is induced by soft excitations is compensated by the repulsive Lee-Huang-Yang corrections stemming from quantum fluctuations of hard modes. The arrested collapse results in self-bound filament-like droplets which is a novel feature of dipolar systems in general as it is directly linked to the anisotropic nature of the DDI. Hence, this analysis is crucial for future experiments with dipolar gases.

Recent Monte Carlo simulations [150] and the dependence of the three-body losses revealed that the LHY term is indeed the stabilization mechanism [77].

In section 5.1 we derive the equation of motion for the condensate wave function including the effects of quantum fluctuations. This is applied in section 5.2 to the experimental relevant case of quench experiments in dysprosium. Section 5.3 is dedicated to the properties of the individual droplets and in section 5.4 we compare the experimentally measured statistics and lifetimes with theoretical predictions. We conclude the chapter in section 5.5.

\subsection{Framework}

We consider a dipolar BEC in the weak interacting limit so that a mean-field description can be applied. As discussed in section 4.3 the existence of quantum fluctuations leads to a shift of the chemical potential that reads (see also Eq. (4.22))

$$
\Delta \mu\left(n, \epsilon_{\mathrm{dd}}\right)=\frac{32}{3 \sqrt{\pi}} g n \sqrt{n a_{s}^{3}} F\left(\epsilon_{\mathrm{dd}}\right),
$$

where the function $F\left(\epsilon_{\mathrm{dd}}\right)$ characterizes the dipolar shift of the LHY correction relative to a purely contact interacting gas as discussed in section 4.3. It becomes 
complex for $\epsilon_{\mathrm{dd}}>1$ signaling a dynamical instability as the mean-field terms, i.e. contact and dipolar interaction together, become on average attractive. In the vicinity of the instability, $\epsilon_{\mathrm{dd}} \sim 1$, the overwhelming contribution to $F\left(\epsilon_{\mathrm{dd}}\right)$ stems from hard modes. Crucially, this is true even when the BEC becomes unstable so that the LHY term is correctly described by Eq. (5.1) even for slightly mean-field unstable systems. The small contribution of the soft modes manifests itself as the small imaginary part of $F\left(\epsilon_{\mathrm{dd}}\right)$ typically orders of magnitude smaller than its real part. This resembles the situation of Bose-Bose mixtures discussed in section 4.2. Let us discuss these similarities in detail in order to explain the key ingredients.

Both systems contain more than one mean-field contribution which cancel each other almost completely close to the stability threshold. Moreover, both systems contain soft modes driving the instability and hard modes which exert a repulsive force with a stronger density scaling than the mean-field term. Thus, the LHY term plays a crucial role close to instability as the residual attractive mean-field interaction can be compensated even by a small LHY correction. Therefore, as in the case of the Bose-Bose mixture we can neglect the contribution of the unstable modes in the LHY term for $\epsilon_{\mathrm{dd}} \sim 1$ so that the contribution of the hard modes results in a repulsive LHY correction $\propto n^{3 / 2}$.

Let us consider at this point a three-dimensionally harmonically trapped BEC, $V_{\mathrm{tr}}(\mathbf{r})=\frac{M}{2}\left(\omega_{x}^{2} x^{2}+\omega_{y}^{2} y^{2}+\omega_{z}^{2} z^{2}\right)$. We evaluate the effect of quantum fluctuations by treating the excitations quasiclassically and employing the LDA as discussed in section (4.3) so that we obtain a corrected equation of state which we insert into a generalized non-linear non-local Schrödinger equation

$$
\begin{aligned}
\mathrm{i} \hbar \frac{\partial \psi(\mathbf{r}, t)}{\partial t}= & {\left[-\frac{\hbar^{2} \nabla^{2}}{2 M}+V_{\mathrm{tr}}(\mathbf{r})+g n(\mathbf{r}, t)+\int d^{3} r^{\prime} V_{\mathrm{dd}}\left(\mathbf{r}-\mathbf{r}^{\prime}\right) n\left(\mathbf{r}^{\prime}, t\right)\right.} \\
& \left.+\frac{32}{3 \sqrt{\pi}} g n(\mathbf{r}, t) \sqrt{n(\mathbf{r}, t) a_{s}^{3}} F\left(\epsilon_{\mathrm{dd}}\right)\right] \psi(\mathbf{r}, t) .
\end{aligned}
$$

This equation is appealing since it allows for a simplified analysis of the effects of quantum fluctuations. However, the use of the LDA must be carefully considered. As discussed in section 4.4 the local density approximation can be safely applied if the droplets are in the TF regime. All droplets discussed below are in the TF regime along $z$, whereas only large droplets are in the TF regime also along $\mathrm{x}$ and $\mathrm{y}$. For small droplets, with less than 4000 atoms, the xy density profile approaches rather a Gaussian form. We discuss the validity of the LHY term for these small droplets in appendix A showing that even in this case the major contribution to the LHY corrections stems from hard modes whose wavelength is small enough to be described well within the LDA.

\subsection{Quench Experiments}

In order to compare our results with recent experiments [76] we consider a BEC with $N$ dysprosium atoms within a three-dimensional harmonic trap with frequencies $\omega_{x, y, z} /(2 \pi)=(44,46,133) \mathrm{Hz}$. We employ an imaginary-time evolution of Eq. (5.2) to form an initial BEC with $a_{s}=120 a_{B}$. Under these conditions, the $\mathrm{BEC}$, with the ground state wave function $\psi_{0}$, is stable and in the TF regime. ${ }^{1}$ At

\footnotetext{
${ }^{1}$ Albeit the dipole length $a_{\mathrm{dd}}=132 a_{B}$ is larger than the scattering length the condensate is clearly stable due to being confined in a pancake-shaped trap (see section 2.2).
} 
finite temperature $T$, thermal fluctuations seed the modulational instability after the quench of the scattering length discussed below, and may influence droplet nucleation. Following Ref. [148], we add thermal fluctuations (for $T=20 \mathrm{nK}$ ) in the form $\psi(\mathbf{r}, t=0)=\psi_{0}(\mathbf{r})+\sum_{n} \alpha_{n} \phi_{n}$, where $\phi_{n}$ are the eigenmodes of the harmonic trap with eigenenergies $\epsilon_{n}$. The sum is restricted to $\epsilon_{n}<2 k_{B} T$, and $\alpha_{n}$ is a complex Gaussian random variable with $\left\langle\left|\alpha_{n}\right|^{2}\right\rangle=\frac{1}{2}+\left(e^{\epsilon_{n} /\left(k_{B} T\right)}-1\right)^{-1}$.
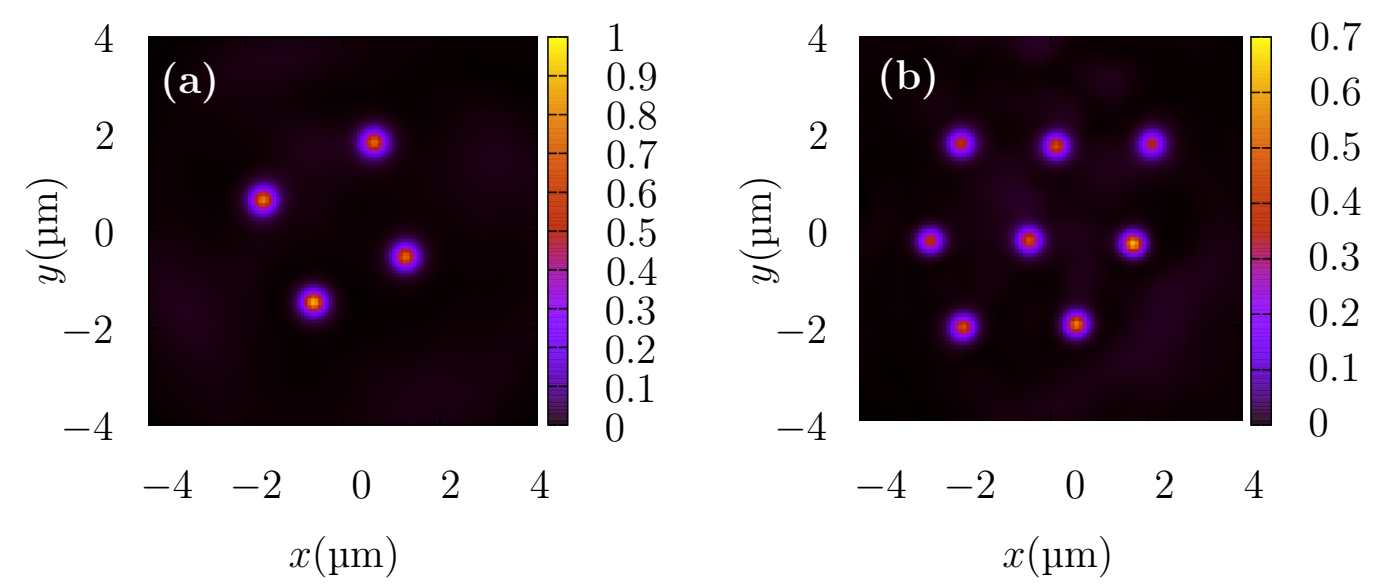

Figure 5.1.: Crystal-like droplet arrangements of $n_{X Y}(x, y) / N$, with $n_{X Y}(x, y)=$ $\int d z n(\mathbf{r})$, for a BEC of $N=7500$ atoms (a), and 15000 atoms (b), initially formed with $a_{s}=120 a_{B}, 20 \mathrm{~ms}$ after a quench to $a_{s}=70 a_{B}$.

At $t=0$ we perform a quench of the scattering length $a_{s}$ in $0.5 \mathrm{~ms}$ to a final value of $a_{s}=70 a_{B}$ that destabilizes the BEC. This value is chosen to ensure that even small condensates are destabilized as the threshold depends on the number of particles and quenches to $a_{s}=80 a_{B}$ destabilize only condensates with $N \gtrsim 1 \times 10^{4}$. The most unstable Bogoliubov mode has a nonzero angular momentum. As a result, the BEC develops at zero temperature $T=0$ an initial ring-like modulational instability on the xy plane, followed by azimuthal symmetry breaking into droplets. Droplets may be nucleated from thermal fluctuations at finite temperature before the ring develops. In both cases stable droplets form within a few ms, which arrange in a quasi-crystalline structure as can be seen in Fig. 5.1, in excellent agreement with Ref. [76]. A residual dynamics remains in the crystal structure leading to a periodically expansion and contraction of the structure exhibiting a constant conversion of energy between kinetic, potential and interdroplet DDI energy. However, droplet nucleation does not involve the whole condensate. A significant number of atoms remains in a halo-like background too dilute to gather particles into stable droplets which makes up approximately $30 \%$ of all particles in the system and is barely visible in Fig. 5.1 due to the contrast.

\subsection{Properties of a single droplet}

The stable quantum filaments result from the compensation at large enough densities of the attractive mean-field terms, $\mu_{\mathrm{MF}} \propto n(\mathbf{r})$, by the effective repulsion introduced by the LHY corrections, $\Delta \mu \propto n(\mathbf{r})^{3 / 2}$. This clearly distinguishes them from solitons, which are stabilized by the kinetic term. Note that this occurs even for a small condensate depletion because the mean-field term is characterized by 
an almost complete cancellation of the short-range and dipolar contributions. In order to study the properties of individual droplets, we evolve Eq. (5.2) in imaginary time for $a_{s}=70 a_{B}$. In order to guarantee the formation of a single droplet in our numerics, we employ as an initial condition for the imaginary-time evolution an elongated Gaussian wave function at the trap center very compressed on the xy plane. Using other initial conditions, in particular a pancake wavefunction elongated on the xy plane, results in the formation of variable droplet configurations similar to those discussed in the quench dynamics. This shows that droplet nucleation and the formation of (metastable) droplet structures should occur not only in the post-quench dynamics, but also when directly forming the condensates at sufficiently low scattering lengths, as discussed in Ref. [76].

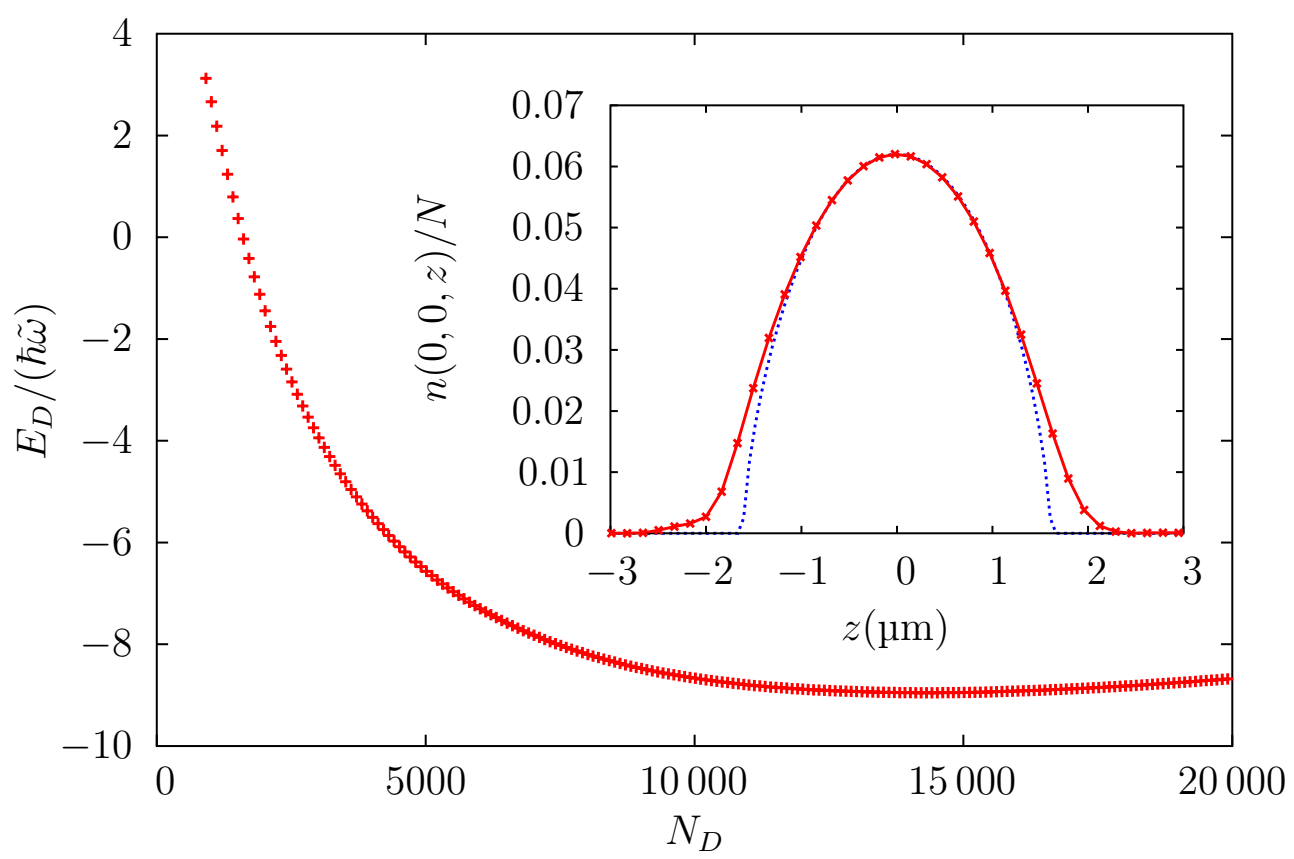

Figure 5.2.: Droplet energy per particle $E_{D}$ in units of $\hbar \tilde{\omega}$ as a function of the number of atoms in the droplet $N_{D}$ for a scattering length of $a_{s}=$ $70 a_{B}$. No droplet solution can be found for particle numbers below the critical value $N_{\min } \simeq 900$. Droplets with a positive energy $E_{D}>0$ occur only in a small window $900 \lesssim N_{D} \lesssim 1500$ of particle numbers. In the inset, we show the density profile (red solid line with crosses) of a droplet with $N_{D}=1000$ at the trap center for the cut $x=y=0$. At the droplet center, $n(0,0, z) \propto\left(1-z^{2} / Z^{2}\right)^{2 / 3}$ (blue dotted curve).

Figure 5.2 shows the droplet energy per particle $E_{D}$ (including the LHY correction) as a function of the number of particles in the droplet $N_{D}$. Two important features are worth mentioning. There is a minimal particle number, $N_{\min } \simeq 900$, such that for $N_{D}<N_{\min }$ no stable droplet can be formed. If the local density does not allow for the gathering of that critical number, then no droplet is formed, accounting for the background halo. Second, $E_{D}\left(N_{D}\right)$ presents a non-monotonous dependence with $N_{D}$, showing a minimum at $N_{D} \simeq 13000$. Moreover, the energy is only positive at small particle numbers close to $N_{\min }$ whereas the energy per particle for large droplets is largely negative. This is particularly relevant for droplet nucleation in quench experiments. The BEC energy, which after the 

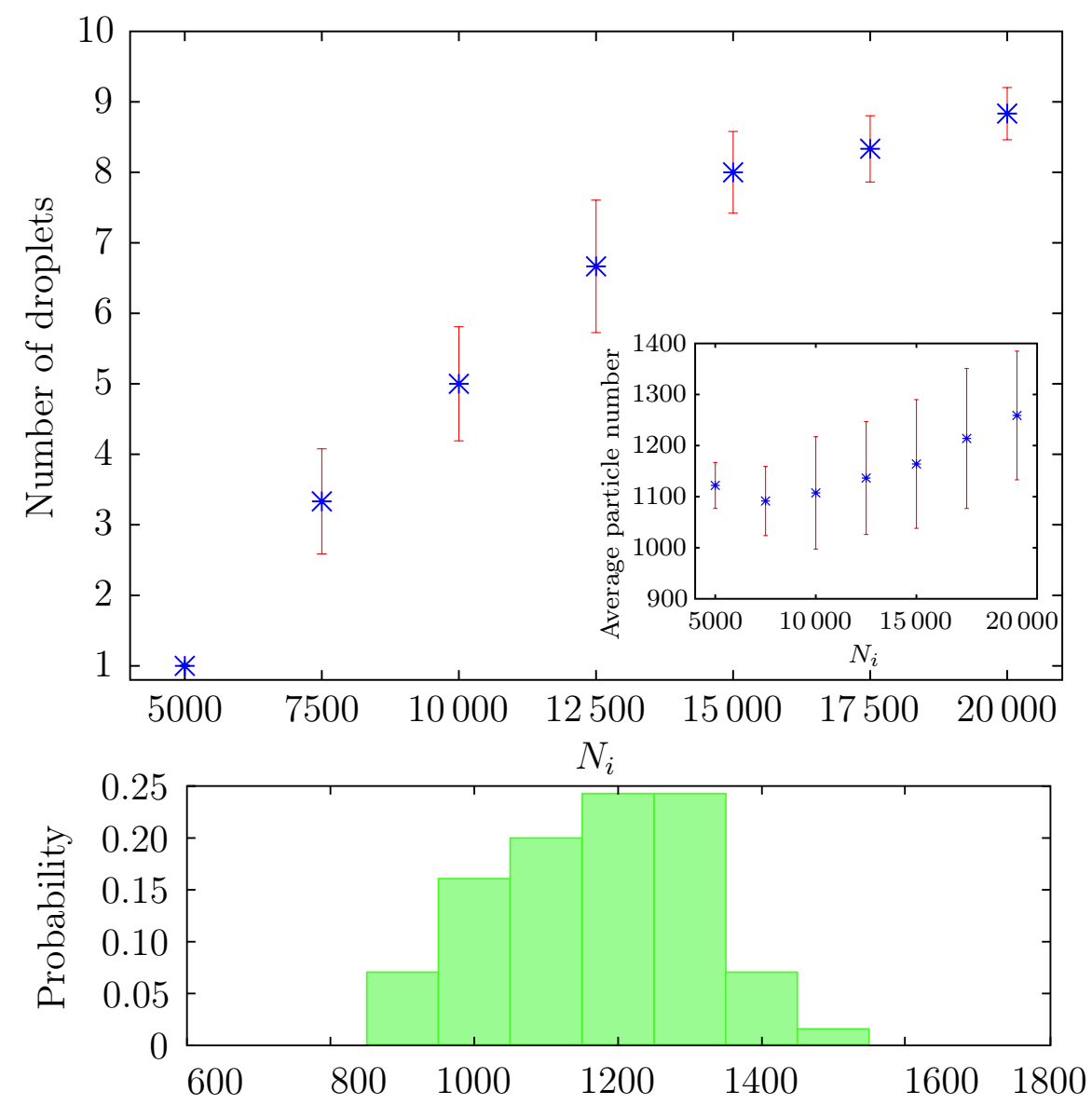

Number of particles in the droplet

Figure 5.3.: (a) Number of droplets as a function of the initial atom number $N_{i}$ $20 \mathrm{~ms}$ after a quench from $a_{s}=120 a_{B}$ to $70 a_{B}$; inset: atom number per droplet as a function of the initial atom number $N_{i}$ under the same conditions. In both cases the average is denoted by a blue cross, and the variance by the error bar; (b) Histogram of the atom number per droplet for the same conditions evaluated from a sample of 260 droplets.

quench is initially positive ( $\simeq 8 \hbar \tilde{\omega}$ in Figs. 5.1), is almost conserved during the droplet formation, just decreasing in a much longer time scale due to three-body losses. The final droplet gas is characterized by the internal energy of the droplets, the center of mass energy of the droplets, and the inter-droplet repulsive DDI. The much more dilute halo has a comparatively small contribution. Although the center of mass energy and the repulsive inter-droplet interaction are obviously positive, they cannot balance a negative internal energy of the droplets, as required by the quasi-conservation of the energy mentioned above. Thereby the number of atoms in the nucleated droplets lies in the narrow window of atom numbers between 900 and 1500, despite the fact that bigger droplets are in fact stable (Fig. 5.2).

The droplets are markedly elongated along the $z$-direction exhibiting a clear cigar shape. The typical size of droplets in the crystal-like structure is $\simeq 4 \mu \mathrm{m}$ in $z$-direction, whereas along the $x$ - and $y$-direction it is $\lesssim 0.6 \mu \mathrm{m}$. This elongation 


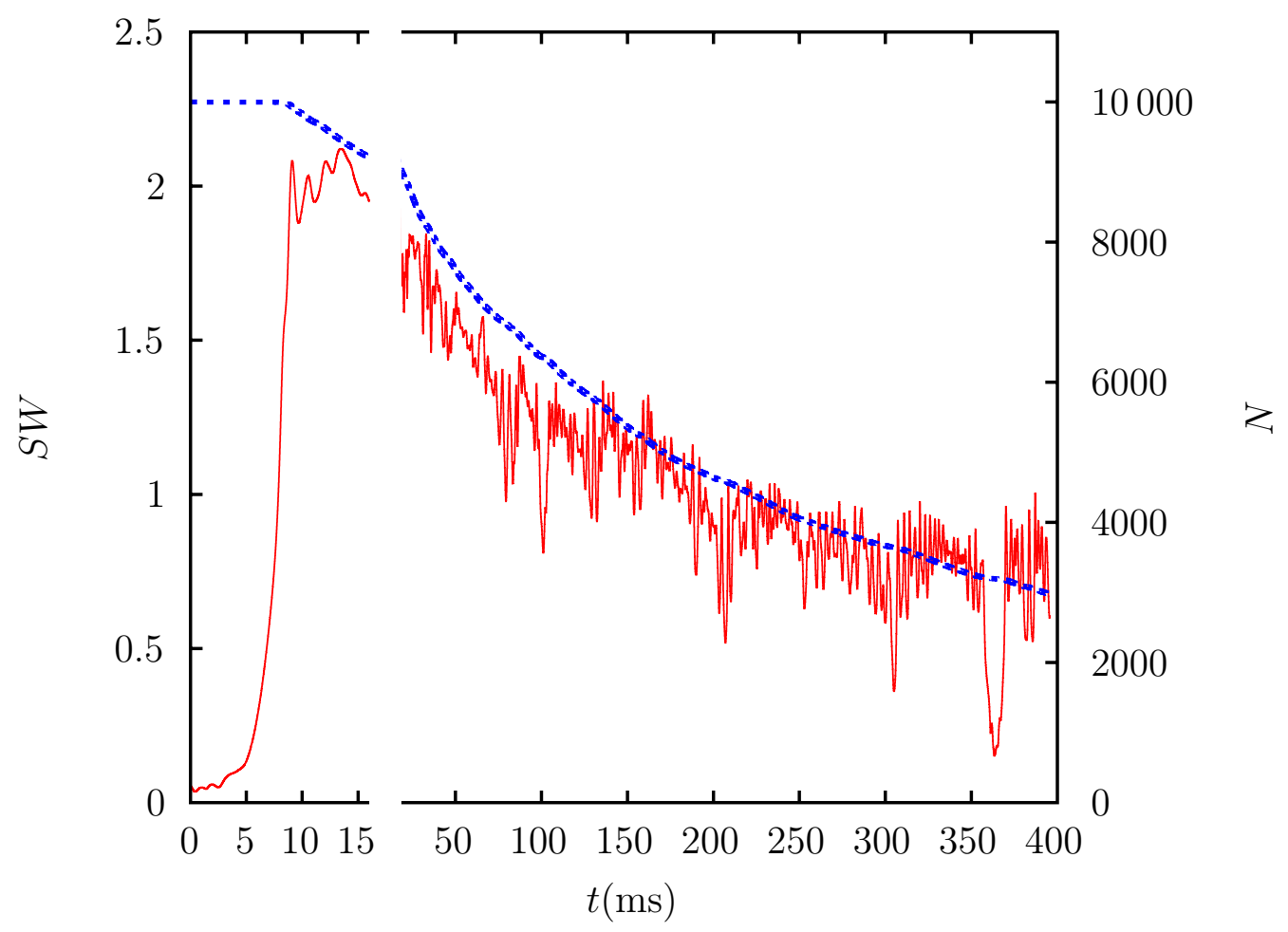

Figure 5.4.: Number of atoms $N$ (blue dotted line) and spectral weight $S W$ (red solid line) as a function of the time after a quench from $a_{s}=120 a_{B}$ to $70 a_{B}$ for a BEC with initially $10^{4}$ atoms. The figure shows a fast creation of the droplet crystal within $10 \mathrm{~ms}$ followed by a slow decay due to three-body losses. The residual dynamics can be clearly seen from the variations of the spectral weight.

is required for the attractive DDI to overwhelm the repulsive contact mean-field term. The droplets are in any case in the Thomas Fermi regime along $z$. Droplets with $N_{D}>8000$, are also TF along xy, whereas those with $N_{D}<4000$ are approximately Gaussian. The latter is also the case for the droplets that are formed in real time after a quench. Note that due to the LHY term, the droplets do not present an inverted-paraboloid profile even in the TF regime, but rather $n(x=0, y=0, z) \sim\left(1-z^{2} / Z^{2}\right)^{2 / 3}$ (inset of Fig. 5.2). Quantum pressure is hence non-negligible for small droplets, but it is not crucial for the droplet stability, which is provided by the compensation of the attractive mean-field interaction and the LHY correction. This is in stark contrast with bright solitons, which result from the compensation of quantum pressure and attractive interactions $[151,152]$.

\subsection{Statistics of the droplet cloud}

With the basic properties of a single droplet at hand we return to the study of the droplet crystal-like structures created in the quench experiments in pancake geometries. We have performed for different initial atom number $N_{i}$ simulations of the BEC dynamics after the quench of the scattering lengths $a_{s}$, starting from different initial conditions given by random thermal fluctuations as explained in section 5.2. We integrated the density over a three-dimensional box which fully 
contains the droplet in order to extract the number of atoms in a droplet. It varies from droplet to droplet in a single realization and between realizations as in the actual experiments, since stable droplets may be formed for different $N_{D}>N_{\text {min }}$. The variance of the number of droplets is additionally affected by the variable importance of the background halo. In addition, droplets formed with a small number of atoms close to the boundary of instability $N_{D} \simeq N_{\min }$, may become unstable against melting in the halo. Considering three-body losses leads therefore to a change in the number of droplets in time. In order to determine objectively the number of droplets, we calculate the column density, $n_{X Y}(x, y) \equiv$ $\int d z n(\mathbf{r})$, after $20 \mathrm{~ms}$ of post-instability dynamics and define a local peak to be a droplet if it satisfies $n_{X, Y} / N>0.3$ (see e.g. Figs. 5.1). Figure 5.3(a) summarizes our results. The droplet number shows an approximate linear dependence with $N_{i}$, in agreement with experiments [76]. The deviation at larger $N_{i}$ occurs because the droplet nucleation for condensates with a large initial atom number is not finished for large $N_{i}$ after $20 \mathrm{~ms}$. The deviation at low $N_{i}$ is due to the longer time needed to develop droplets as the initial condensate is more stable due to the larger effect of the repulsive quantum pressure (e.g. $N_{i}=5000$ develops up to 3 droplets at $t \sim 40 \mathrm{~ms}$ ). The approximate linear dependence of Fig. 5.3(a) stems from the local character of the nucleation, which results in a particle number per droplet basically independent of the initial number of atoms $N_{i}$ (inset of Fig. 5.3(a)). The histogram of Fig. 5.3(b) shows that, as expected from our discussion of the droplet energy, the particle number per droplet lies between 900 and 1500, with an average of approximately 1200, again in good agreement with experiments.

We obtain peak densities of $\sim 2 \times 10^{21} \mathrm{~m}^{-3}$. At those densities three-body losses become relevant in the long run. We take them into account by adding a loss term to the right hand side of the non-linear non-local Schrödinger equation as discussed in section 1.5 yielding

$$
\begin{aligned}
\mathrm{i} \hbar \frac{\partial \psi(\mathbf{r}, t)}{\partial t}= & {\left[-\frac{\hbar^{2} \nabla^{2}}{2 M}+V_{\mathrm{tr}}(\mathbf{r})+g n(\mathbf{r}, t)+\int d^{3} r^{\prime} V_{\mathrm{dd}}\left(\mathbf{r}-\mathbf{r}^{\prime}\right) n\left(\mathbf{r}^{\prime}, t\right)\right.} \\
& \left.+\frac{32}{3 \sqrt{\pi}} g n(\mathbf{r}, t) \sqrt{n(\mathbf{r}, t) a_{s}^{3}} F\left(\epsilon_{\mathrm{dd}}\right)-\mathrm{i} \hbar \frac{L_{3}}{2} n(\mathbf{r}, t)^{2}\right] \psi(\mathbf{r}, t) .
\end{aligned}
$$

The exact value of the three-body loss coefficient is not yet known, but as stressed in Ref. [153] it should be in the lower $10^{-41} \mathrm{~m}^{6} / \mathrm{s}$. Therefore we consider as threebody loss rate a value of $L_{3}=1.2 \times 10^{-41} \mathrm{~m}^{6} / \mathrm{s}$. Figure 5.4 shows the time dependence of the atom number and of the spectral weight, $S W=\int d^{2} k \tilde{n}_{X Y}(\mathbf{k})$, where $\tilde{n}_{X Y}(\mathbf{k})$ is the Fourier transform of $n_{X Y}(x, y)$ and the integral extends from $k_{\min }=1.5 \mu^{-1}$ to $k_{\max }=5 \mu^{-1}$. The spectral weight characterizes the appearance (and disappearance) of the droplet pattern [76]. The losses not only decrease the atom number, but also lead to the destruction of droplets, which may lose too much particles to remain stable against melting in the background. Moreover, the slow energy dissipation induced by three-body losses at the BEC maxima is relevant for the formation of droplet arrangements (Fig. 5.1) that minimizes inter-droplet repulsion. Figure 5.4 shows a growth of $S W$ up to $t \sim 10 \mathrm{~ms}$, and a decrease in a much longer time scale of several hundreds of ms, accompanied by the corresponding atom loss, in excellent agreement with experiments. 


\subsection{Conclusion}

In summary, quantum fluctuations can prevent the collapse of unstable dipolar BECs which leads to the formation of stable quantum filaments as experimentally observed in recent dysprosium experiments [76]. Since the LHY correction depends crucially on the factor $n a_{s}^{3}$, we expect that droplets should collapse for lower scattering lengths which provides a criterion to discern LHY stabilization from stabilization based on three-body forces [148,149]. Other ways of discerning between the two stabilization mechanisms are discussed in the next chapter. Our results, which are based on a generalized non-linear non-local Schrödinger equation, are already in very good agreement with experiments [76], although a more precise analysis of long-wave length excitations in small droplets may be necessary to provide a fully quantitative comparison. This is particularly true for Dy experiments, whereas for Er experiments discussed in chapter 7 the quantitative agreement is even better. We stress that LHY stabilization results from the anisotropy of the DDI and is therefore a general effect in strongly dipolar BECs. It can not be found in experiments with chromium experiments [70] because of the much weaker dipole-dipole interactions leading to a smaller contact term at the stability threshold and therefore also to smaller LHY corrections (see Eq. (5.1)). As a chromium BEC becomes unstable at a scattering length value $\sim 10$ times smaller than in dysprosium quantum stabilization would demand densities at least a factor of $10^{3}$ times larger than in Dy due to the $n a_{s}^{3}$ dependence of the LHY term (see chapter 6 for a precise study of the density dependence). This yields densities in the regime of $\sim 10^{24} \mathrm{~m}^{3}$, which is unreachable due to three-body losses. In contrast, LHY stabilization and droplet nucleation are a characteristic general feature induced by the DDI that should play a key role in other future experiments with strongly dipolar gases of highly-magnetic atoms (see chapter 7) and polar molecules. 


\section{Ground-state properties and elementary excitations of quantum filaments}

In the previous chapter we showed that the repulsive LHY term can stabilize the collapse of a dipolar BEC. We investigated the formation of stable quantum droplets and showed a qualitative and quantitative understanding of recent experimental results [76]. In this chapter we investigate theoretically the properties of a single quantum droplet in detail by discussing the different ground-state solutions, the collective oscillations, as well as the existence and properties of self-bound droplets. This will be important for future experiments. We introduce the framework in section 6.1 and discuss the properties of the ground state in section 6.2. Section 6.3 is dedicated to self-bound solutions followed by a discussion of the lowest-lying collective oscillations in section 6.4. We conclude the chapter in section 6.5.

\subsection{Framework}

As in chapter 5 we consider a harmonically trapped BEC of $N$ dipoles oriented along the $z$-direction by an external magnetic field. We describe the system by the non-linear non-local Schrödinger equation (5.2) as discussed in section 5.1. We note that the use of Eq. (5.2) is just valid for cigar-shape traps or moderately pancake ones. This stems from the fact that Eq. (5.2) is only valid in the meanfield unstable regime of $\epsilon_{\mathrm{dd}}$ close to 1, i.e. the instability threshold for a 3D homogeneous dipolar BEC. For traps with larger aspect ratio the trap geometry significantly stabilizes the BEC. As a result, the stability threshold occurs for much lower (or even negative) scattering lengths [70], departing significantly from the $\epsilon_{d d}=1$ value. In that case the use of known results for homogeneous 3D BECs becomes unjustified. Due to this reason in all calculations below we restrict our analysis to aspect ratios $\lambda \leq 3$.

Additionally, we employ a Gaussian ansatz in order to gain more insight in the droplet physics [63]

$$
\psi(x, y, z)=\frac{1}{\pi^{\frac{3}{4}}\left(w_{x} w_{y} w_{z}\right)^{\frac{1}{2}}} \prod_{\eta=x, y, z} \mathrm{e}^{-\frac{\eta^{2}}{2 w_{\eta}^{2}}+i \eta^{2} \beta_{\eta}(t)},
$$

which provides remarkably good results especially taking into account that the droplets discussed below are in the $\mathrm{TF}$ regime. The variational parameters are the widths $w_{\eta}$ in the $\eta=x, y, z$ direction, and $\beta_{\eta}$, which determines the phase curvature along $\eta$. Compared to [63] we do not consider the possibility of the 
sloshing mode of the condensate. The Lagrangian density reads

$$
\begin{aligned}
\mathcal{L}= & \frac{\mathrm{i} \hbar}{2}\left(\psi \frac{\partial \psi^{*}(\mathbf{r}, t)}{\partial t}-\psi^{*} \frac{\partial \psi(\mathbf{r}, t)}{\partial t}\right)+\frac{\hbar^{2}}{2 M}|\nabla \psi(\mathbf{r}, t)|^{2} \\
& +V_{\mathrm{tr}}(\mathbf{r})|\psi(\mathbf{r}, t)|^{2}+\frac{g}{2}|\psi(\mathbf{r}, t)|^{4}+\frac{2}{5} g_{\mathrm{LHY}}|\psi(\mathbf{r}, t)|^{5} \\
& +\frac{1}{2} \int d^{3} r^{\prime} V_{\mathrm{dd}}\left(\mathbf{r}-\mathbf{r}^{\prime}\right)|\psi(\mathbf{r}, t)|^{2}\left|\psi\left(\mathbf{r}^{\prime}, t\right)\right|^{2}
\end{aligned}
$$

where $g_{\mathrm{LHY}}=\frac{32}{3 \sqrt{\pi}} g \sqrt{N a_{s}^{3}} F\left(\epsilon_{\mathrm{dd}}\right)$ characterizes the strength of the LHY corrections. We insert the ansatz (6.1) into (6.2), obtain the Lagrangian $L=\int d^{3} r \mathcal{L}$, and establish the corresponding Euler-Lagrange equations:

$$
\beta_{\eta}=\frac{M}{2 \hbar w_{\eta}} \frac{d w_{\eta}}{d t}
$$

and

$$
\frac{d^{2} v_{j}}{d \tau^{2}}=-\frac{\partial}{\partial v_{j}} U\left(v_{x}, v_{y}, v_{z}\right)
$$

In the latter equation, we have employed dimensionless units $\tau=\tilde{\omega} t, w_{\eta}=\tilde{l} v_{\eta}$, $\tilde{l}=\sqrt{\hbar /(M \tilde{\omega})}$, with $\tilde{\omega}=\left(\prod_{\eta} \omega_{\eta}\right)^{1 / 3}$, and introduced the effective potential:

$$
U=\frac{1}{2} \sum_{\eta}\left[v_{\eta}^{-2}+\left(\frac{\omega_{\eta}}{\tilde{\omega}}\right)^{2} v_{\eta}^{2}\right]+\frac{2}{3} \frac{P Q}{\left(\prod_{\eta} v_{\eta}\right)^{\frac{3}{2}}}+\frac{P}{\prod_{\eta} v_{\eta}}\left[1+\epsilon_{d d} F\left(\frac{w_{z}}{w_{x}}, \frac{w_{z}}{w_{y}}\right)\right],
$$

where

$$
F\left(\kappa_{x}, \kappa_{y}\right)=\frac{1}{4 \pi} \int_{0}^{\pi} d \theta \sin \theta \int_{0}^{2 \pi} d \phi\left[\frac{3 \cos ^{2} \theta}{\left(\kappa_{x}^{2} \cos ^{2} \phi+\kappa_{y}^{2} \sin ^{2} \phi\right) \sin ^{2} \theta+\cos ^{2} \theta}-1\right] .
$$

The dimensionless constants $P=\sqrt{\frac{2}{\pi}} \frac{N a_{s}}{\tilde{l}}$ and $Q=\frac{512 F\left(\epsilon_{d d}\right)}{25 \sqrt{5} \pi^{\frac{5}{4}}} \sqrt{N \frac{a_{s}^{3}}{\tilde{l}^{3}}}$ characterize, respectively, the strength of the contact interaction and the LHY correction.

The equilibrium widths $v_{i 0}$ are calculated by minimizing the potential $U$. In addition, the low-lying excitations around the equilibrium are determined by evaluating the Hessian matrix $M_{\eta \eta^{\prime}}=\frac{\partial^{2} U}{\partial v_{\eta} \partial v_{\eta^{\prime}}}$ at the minimum. We consider in this chapter for simplicity a cylindrical trap, $\omega_{x}=\omega_{y}=\omega_{z} / \lambda$. In that case, the lowest excitation frequencies are:

$$
\begin{aligned}
\omega_{1}^{2}= & M_{x x}-M_{x y} \\
\omega_{2,3}^{2}= & \frac{1}{2}\left(M_{x x}+M_{x y}+M_{z z}\right) \\
& \pm \frac{1}{2} \sqrt{\left(M_{x x}+M_{x y}-M_{z z}\right)^{2}+8 M_{x z}^{2}}
\end{aligned}
$$

The corresponding eigenvectors $\left(\eta_{x}, \eta_{y}, \eta_{z}\right)$ characterize the mode geometry. In particular, a mode with $\operatorname{sign}\left(\eta_{x, y, z}\right)= \pm$ has a 3D monopole character; $\operatorname{sign}\left(\eta_{x, y}\right)=$ \pm and $\operatorname{sign}\left(\eta_{z}\right)=\mp$ a $3 \mathrm{D}$ quadrupolar character. The mode $(1 / \sqrt{2},-1 / \sqrt{2}, 0)$ is a 2D quadrupolar mode on the xy plane. 


\subsection{Ground-state properties}

In this section we discuss the ground-state properties of a dipolar BEC in the presence of the LHY stabilization.

\subsubsection{Droplet versus mean-field solution}

We consider a gas of $N$ dysprosium atoms within a cylinder-symmetric trap with fixed geometric mean frequency $\tilde{\omega} / 2 \pi=70 \mathrm{~Hz}$ which is a similar value as that of recent Dy experiments [76]. For a given trap aspect ratio $\lambda$ we obtain the ground state by imaginary time evolution (ITE) of Eq. (5.2) as discussed in section 5.1. In absence of LHY stabilization, there is a critical scattering length $a_{c r}(\lambda)$, such that for $a_{s}<a_{c r}$ the dipolar BEC becomes unstable against collapse, i.e. no stable (or metastable) ground state exists. In contrast, due to the LHY term, Eq. (5.2) presents for any value of $a_{s}$ and $\lambda$ a ground-state solution which depends crucially on $N, a_{s}$, and $\lambda$.

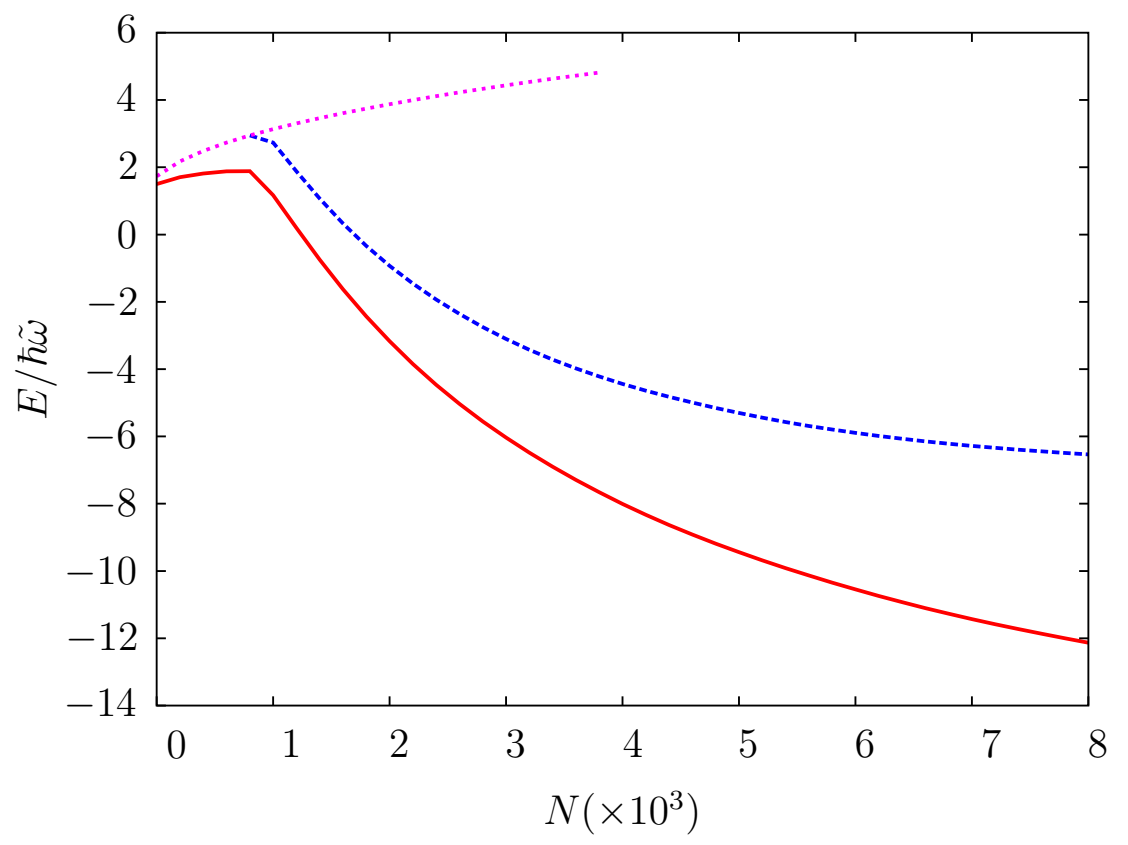

Figure 6.1.: Energy per particle for a Dy BEC with $a_{s}=70 a_{B}$ in a trap with $\tilde{\omega} / 2 \pi=70 \mathrm{~Hz}$ for $\lambda=1$ (red solid line), and for the droplet (blue dashed line) and mean-field (purple dotted line) solutions for $\lambda=3$.

We depict in Fig. 6.1 (red solid curve) the energy per particle as a function of the number of particles $N$ for $a_{s}=70 a_{B}$ and a spherical trap, $\lambda=1$. For small particle numbers $N<1000$ the condensate is stabilized by the quantum pressure and not by the interaction terms or the LHY corrections similar to the case of non-dipolar gases with negative scattering length. In contrast, for $N>1000$ the LHY term is crucial to stabilize the cloud. The condensate becomes elongated along the dipole direction and the BEC energy decreases to markedly negative values. Henceforth we call this elongated solution the droplet solution. Whereas for $\lambda=1$ there is just one possible solution in the ITE of Eq. (5.2), the situation is clearly different for $\lambda=3$. For $N>1000$ the lowest energy solution (blue dashed 


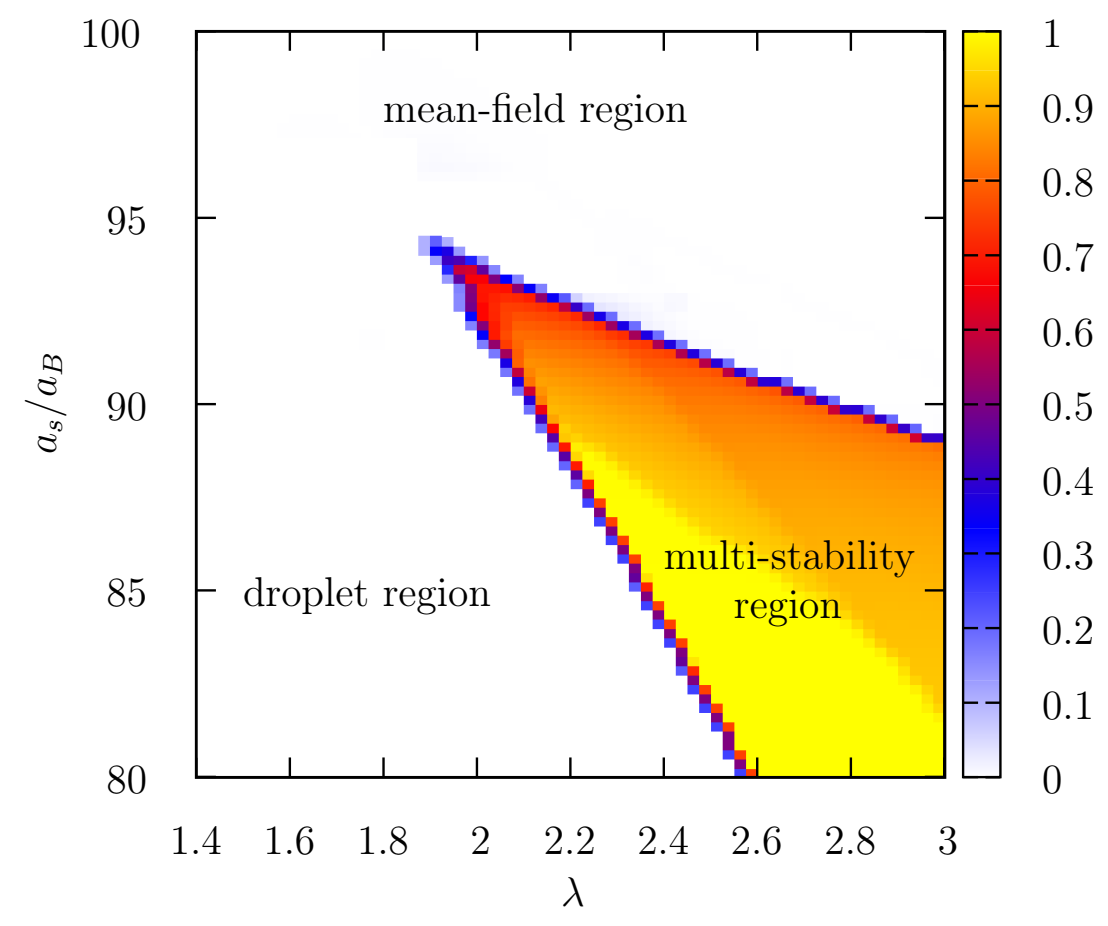

Figure 6.2.: Ground-state phase diagram for $N=20000$ Dy atoms in a cylindrically harmonic trap with $\tilde{\omega} / 2 \pi=70 \mathrm{~Hz}$, as a function of the scattering length $a_{s}$ and the trap aspect ratio $\lambda$. In the multistability region we depict the relative difference between the peak densities of the droplet and the mean-field solutions, $\left|n_{P}^{(D)}-n_{P}^{(M)}\right| /\left(n_{P}^{(D)}+n_{P}^{(M)}\right)$.

curve) is an elongated one with negative energy stabilized by the LHY term, similar to that found for $\lambda=1$. Moreover there is a continuum of metastable multi-droplet states with different numbers of droplets and a variable number of particles. More relevantly, there is a metastable state (purple dotted curve) that connects smoothly with the solution for $N<1000$. This solution has a pancake geometry, being wider on the xy plane than along $z$. The metastable pancake solution exists up to $N \simeq 4000$ and is in contrast to the droplet solution meanfield stable. In order to discern it from the droplet solution, we call this solution the mean-field solution although the LHY term may play already a non-negligible role in its properties.

\subsubsection{Multistability}

The presence of metastable states marks a clear difference between cigar-like and pancake-like traps, which is best illustrated by the dependence of the BEC physics on the scattering length $a_{s}$ and $\lambda$. Figure 6.2 summarizes our results for $N=20000$ Dy atoms. For large scattering length values, the condensate is mean-field stable and does not require LHY stabilization. Then only a single solution (the mean-field solution) exists. For $\lambda<\lambda_{c r}\left(\lambda_{c r} \simeq 1.9\right.$ in Fig. 6.2), there is just one ITE solution that smoothly connects the mean-field and the droplet solution for decreasing scattering length. This smooth crossover results in a characteristic growth of the peak density as shown in section 6.2.3. This 


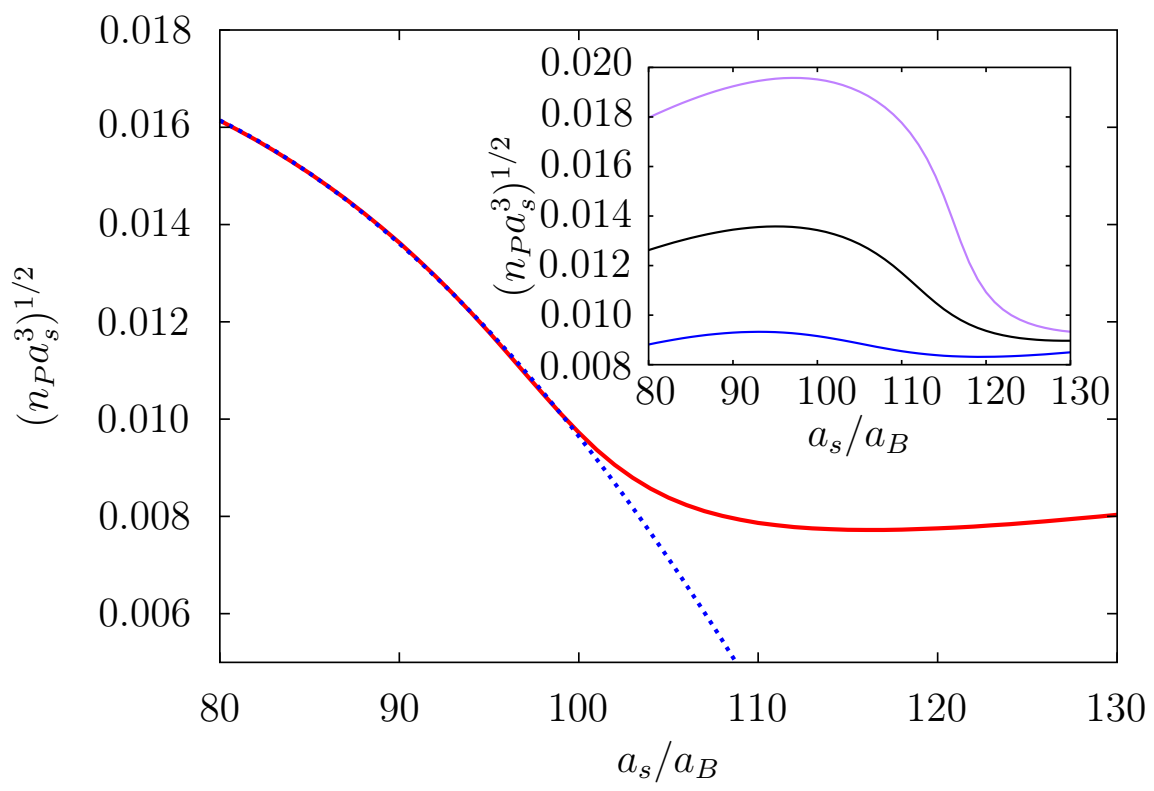

Figure 6.3.: $\left(n_{P} a_{s}^{3}\right)^{1 / 2}$ for a Dy BEC of $N=20000$ in a spherical trap with $\tilde{\omega} / 2 \pi=$ $70 \mathrm{~Hz}$ as a function of the scattering length $a_{s}$. The curve follows in the droplet region the dependence $A\left(1-a_{s} / a_{c}\right)+B\left(1-a_{s} / a_{c}\right)^{2}$ with $A=0.082, B=-0.097$ and $a_{c}=116.48 a_{B}$ (blue dotted curve). In the inset we depict the results obtained for three-body stabilization with (from bottom to top) $g_{3}=2,1$, and $0.5 \times 10^{-38} \mathrm{~m}^{6} / \mathrm{s}$.

regime is particularly relevant for experiments performed in Er as discussed in chapter 7 .

For $\lambda>\lambda_{c r}$ there is a region of $a_{s}$ values where multistability occurs, that separates the mean-field and droplet regions. Within the multistable region the color code in Fig. 6.2 depicts the relative difference $\left|n_{P}^{(D)}-n_{P}^{(M)}\right| /\left(n_{P}^{(D)}+n_{P}^{(M)}\right)$, between the peak densities of the droplet solution, $n_{P}^{(D)}$, and the mean-field solution, $n_{P}(M)$. The lowest border of this region marks the end of the metastability of the mean-field solution. The upper border marks a first order phase transition, characterized by a kink in the chemical potential of the ground-state solution, at which the droplet solution becomes the global energy minimum. A quench through this transition results in the droplet structures discussed in chapter 5 .

\subsubsection{Crossover from the mean-field to the droplet solution}

As mentioned above, for $\lambda<\lambda_{c r}$ there is a smooth crossover for decreasing scattering length $a_{s}$ between the mean-field and the droplet solution marked by a characteristic growth of the peak density. Figure 6.3 shows our results based on ITE of Eq. (5.2) for the peak density as a function of the scattering length $a_{s}$ for $N=20000$ Dy atoms in a spherical trap $(\lambda=1)$. The peak density $n_{P}$ increases dramatically when $a_{s}$ decreases and the BEC enters the droplet region. The dependence of the peak density on $a_{s}$ is markedly different for the LHY and three-body stabilization mechanisms. This difference is more evident when comparing the functional form of $\left(n_{P} a_{s}^{3}\right)^{1 / 2}$. As shown in Fig. 6.3, for the LHY stabilization $\left(n_{P} a_{s}^{3}\right)^{1 / 2}$ follows in the droplet region a characteristic dependence 
$A\left(1-a_{s} / a_{c}\right)+B\left(1-a_{s} / a_{c}\right)^{2}$, with $A, B$, and $a_{c}$ fitting parameters ${ }^{1}$. This functional form fits the peak density well for all trap aspect ratios and particle numbers within the droplet regime. We note as well that the qualitative behavior of the peak-density scaling is also in good agreement with the results obtained from the Gaussian ansatz.

Large conservative three-body interactions have a different density dependence than the LHY term. Therefore a different peak density scaling behavior is expected for a condensate stabilized by three-body interactions leading to a possibility to discern between the two mechanisms. We performed ITE calculations of Eq. (5.2) in which we replaced the LHY term with a three-body term of the form $\frac{\hbar g_{3}}{2}|\psi(\mathbf{r}, t)|^{4} \psi(\mathbf{r}, t)[148,149]$ and extract the peak density as function of the scattering length. The resulting curves of $\sqrt{n_{P} a_{s}^{3}}$ show a clearly non-monotonous behavior irrespective of the value of $g_{3}$ (inset of Fig. 6.3). Hence the analysis of $\sqrt{n_{P} a_{s}^{3}}$ as a function of the scattering length in the crossover regime $\left(\lambda<\lambda_{c r}\right)$ provides a clear way to discern between the two stabilization mechanisms.

Note that the study of three-body losses, which depend crucially on the density, revealed the LHY stabilization mechanism and excluded three-body stabilization mechanism in dysprosium experiments [77].

\subsection{Self-bound condensates}

The interplay of LHY and mean-field terms allows for 3D self-bound (SB) condensates for a sufficiently small scattering length. Figure 6.4(a) shows the boundary between SB and trap-bound solutions. The curve marked by $\times$ symbols is obtained as the point in which the ITE of Eq. (5.2) with $V_{\operatorname{tr}}(\mathbf{r})=0$ results in an unbound solution. The curve marked with + symbols is evaluated from the simplified Gaussian ansatz as the point at which the minimum of the effective potential $U$ disappears. Both curves are in excellent agreement. Note that the boundary is basically vertical for a larger number of particles marking a critical scattering length $a_{\mathrm{SB}}$, such that for $a_{s}>a_{\mathrm{SB}}$ no self-bound solution is possible (for Dy, $\left.a_{\mathrm{SB}} \simeq 120 a_{B}\right)$.

It is important to stress, that within the SB regime, and for a sufficiently large number of particles, the SB droplets are approximately in the TF regime, but obviously they do not present the typical inverted-paraboloid density profile due to the modified equation of state and the absence of harmonic confinement (Figs. 6.4(b) and (c)). This constitutes a clear difference between these SB BECs and bright BEC solitons. The latter are also SB solutions, which however just exist in 1D (or in 2D for dipolar BECs [102]), resulting from the compensation of quantum pressure and attractive mean-field interactions. Hence by definition they cannot exist in the TF regime. Here, in contrast, the droplet remains SB in 3D by the compensation of LHY and mean-field terms, and hence TF SB droplets are allowed, being in fact the general case.

Figure 6.4 shows that there are two non-equivalent ways of entering the SB regime, either by increasing the number of atoms or by decreasing the scattering length $a_{s}$. Figure 6.5 depicts the widths $v_{x, z}^{\mathrm{SB}}$ of the SB BECs. Decreasing $a_{s}$

\footnotetext{
${ }^{1}$ This dependence is expected from the compensation of LHY and mean-field terms, as suggested by Eq. (2) of Ref. [77].
} 

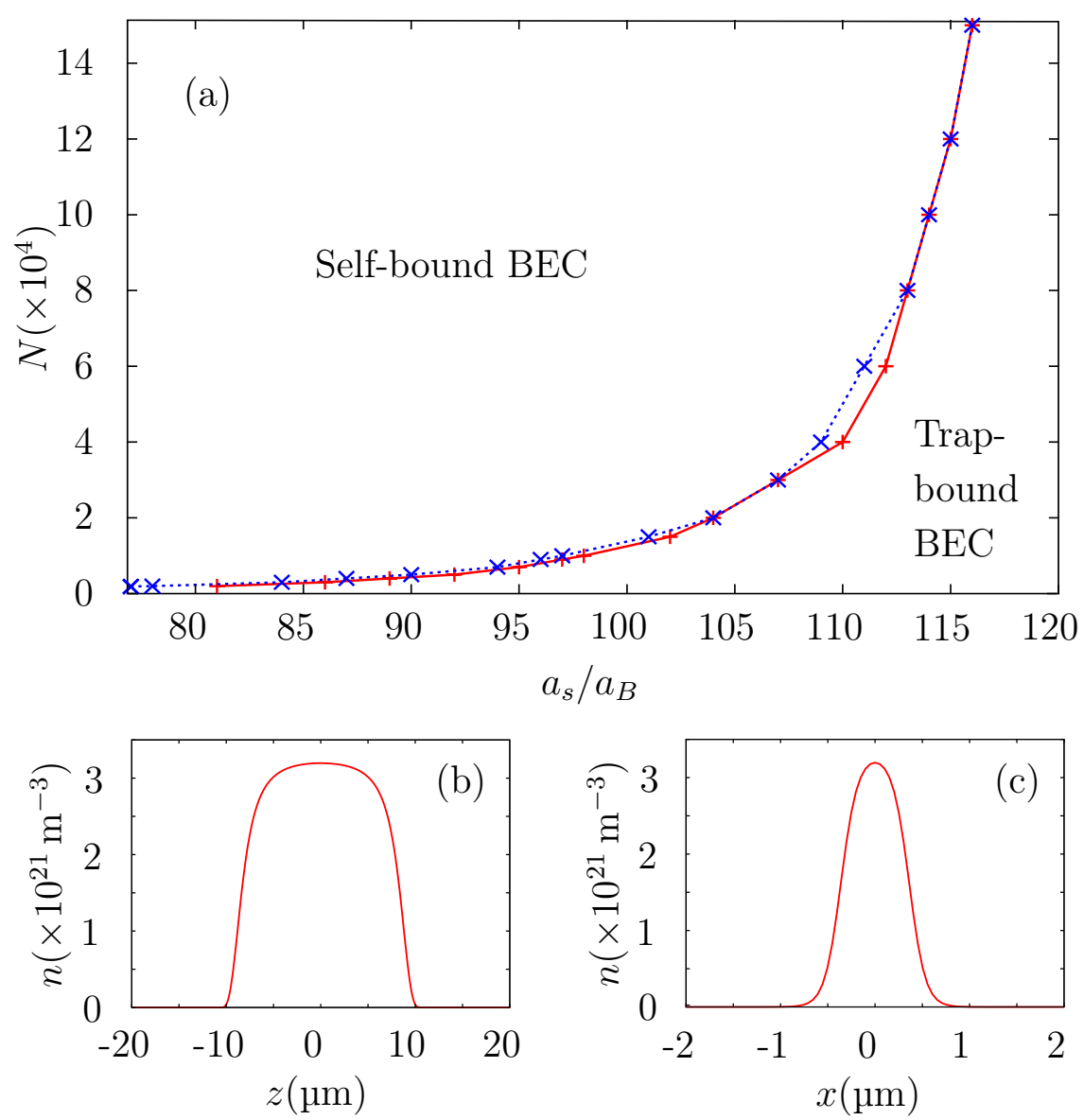

Figure 6.4.: (a) Self-bound and trapped-bound regions for a Dy BEC as a function of the number of particles $N$ and the scattering length $a_{s}$. The figure shows the boundary as calculated from ITE of Eq. $(5.2)(\times)$ and from the variational Gaussian ansatz $(+)$. The lower figures show the density profile along $z$ for $x=y=0$ (b) and along $x$ for $y=z=0$ (c), for a Dy BEC with $N=20000$ and $a_{s}=80 a_{B}$, well within the SB regime.

at constant $N$ leads to a smaller $v_{x, z}^{\mathrm{SB}}$ (Fig. 6.5(b)), although both $v_{z}^{\mathrm{SB}} / v_{x}^{\mathrm{SB}}$ and $n_{P}$ increase significantly. In contrast, increasing $N$ for constant $a_{s}$ results in a rapid increase of $v_{z}^{\mathrm{SB}}$ (Fig. $\left.6.5(\mathrm{a})\right)$, whereas $v_{x}^{\mathrm{SB}}$ remains almost constant, and $n_{P}$ increases. Surprisingly the variational results and those obtained from the direct simulation of Eq. (5.2) are in good agreement, despite the clearly non-Gaussian nature of the BEC deep inside the SB regime. This dependence is relevant for the convergence of the trapped BEC to the SB solution, since as discussed in the following, the convergence of the trapped solution to the SB solution is eased if $v_{z}^{\mathrm{SB}}$ is small. The realization of three-dimensional SB BECs is hence significantly simpler when working at small scattering lengths and low particle numbers.

The trap may significantly alter the properties of the SB solution. Due to the marked elongation of the SB droplets along $z$, the $z$ confinement is particularly crucial. The BEC may depart significantly from the SB solution, if the corresponding oscillator length is smaller than the $z$-width of the self-bound droplet $l_{z}<v_{z}^{\mathrm{SB}}$. This is true not only for the $z$-width of the cloud, but also for the radial one, despite the fact that the condensate is much narrower radially. Figure 6.6 

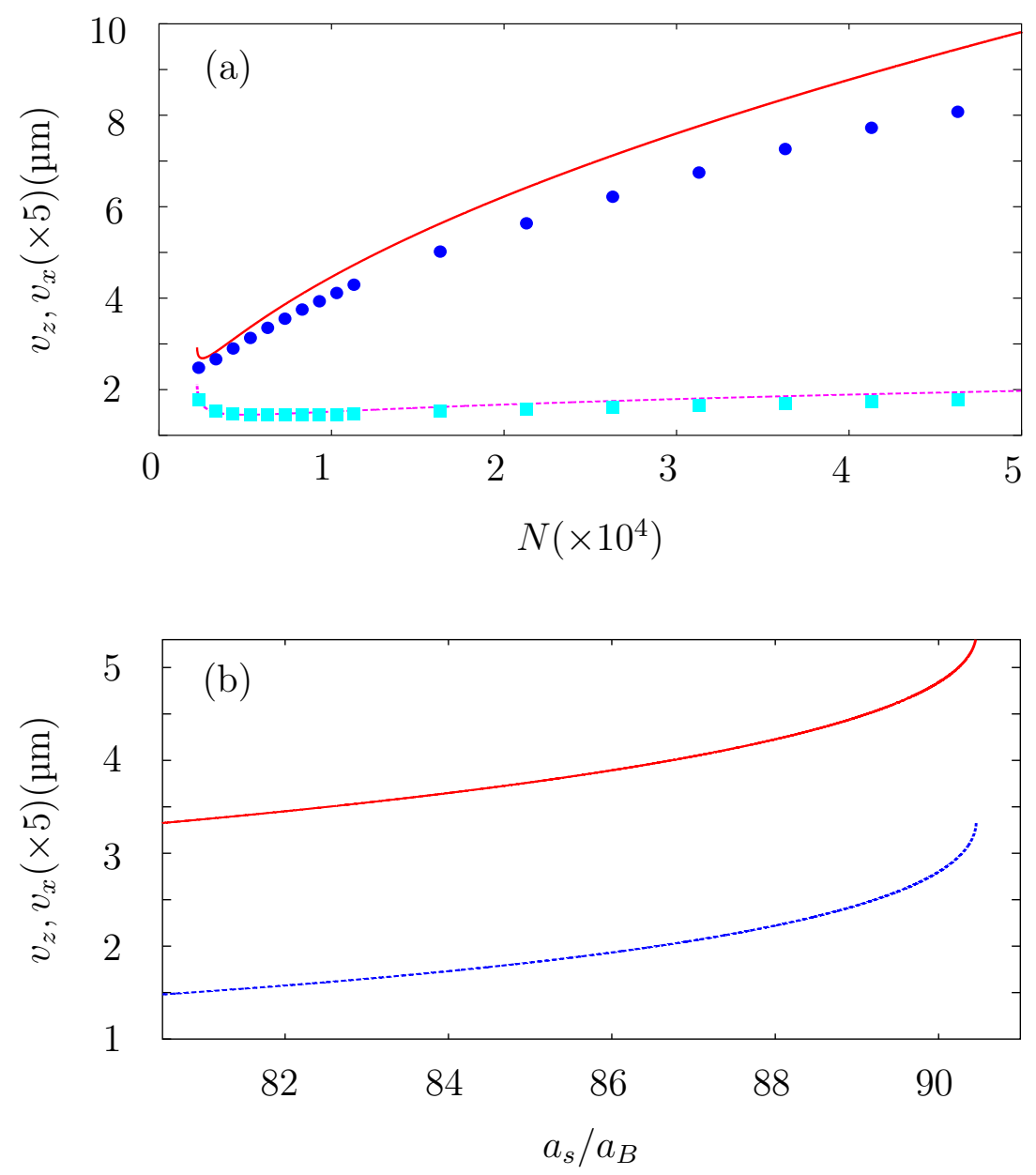

Figure 6.5.: (a) $v_{z}$ (red solid curve) and $v_{x}$ (purple dashed curve) for a SB solution with $a_{s}=80 a_{B}$ as a function of $N$; (b) $v_{z}$ (red solid curve) and $v_{x}$ (blue dashed curve) for a fixed number of particles $N=5000$ as a function of $a_{s}$. The curves depict the results obtained from the variational Gaussian Ansatz. In Fig. (a) we show as well the results obtained from the direct simulation of Eq. (5.2) for $\sqrt{2\left\langle z^{2}\right\rangle}$ (circles) and $\sqrt{2\left\langle x^{2}\right\rangle}$ (squares)

shows our Gaussian ansatz results for $v_{z} / v_{z}^{\mathrm{SB}}$ and $v_{x} / v_{x}^{\mathrm{SB}}$ for $\lambda=1, N=5000 \mathrm{Dy}$ atoms, and $a_{s}=80 a_{B}$, as a function of the ratio $l_{z} / v_{z}^{\mathrm{SB}}$. Convergence demands $l_{z} / v_{z}^{\mathrm{SB}}>1$, which for this case would demand a rather low $\omega_{z} / 2 \pi<5.6 \mathrm{~Hz}$. For typical experimental values of $\tilde{\omega} / 2 \pi=70 \mathrm{~Hz}, v_{z} / v_{z}^{\mathrm{SB}} \simeq 0.8, v_{x} / v_{x}^{\mathrm{SB}} \simeq 1.05$, and $n_{P} / n_{P}^{\mathrm{SB}} \simeq 1.12$, and hence the deviation from the SB solution is relatively small. As discussed above, the realization of the SB solution is much more involved for larger $N$ and $a_{s}$. For example, for $N=20000$ and $a_{s}=100 a_{B}$, for a spherical trap with $\tilde{\omega} / 2 \pi=70 \mathrm{~Hz}, v_{z} / v_{z}^{\mathrm{SB}} \simeq 0.6, v_{x} / v_{x}^{\mathrm{SB}} \simeq 1.2$, and $n_{P} / n_{P}^{\mathrm{SB}} \simeq 1.06$. Typical experiments would hence produce BECs that albeit stabilized by the LHY term may be well away from the 3D SB regime. As a result, abruptly switching off the trap is typically not expected to result in a complete cancellation of the time-of-flight expansion, as one would expect from the 3D SB character. 


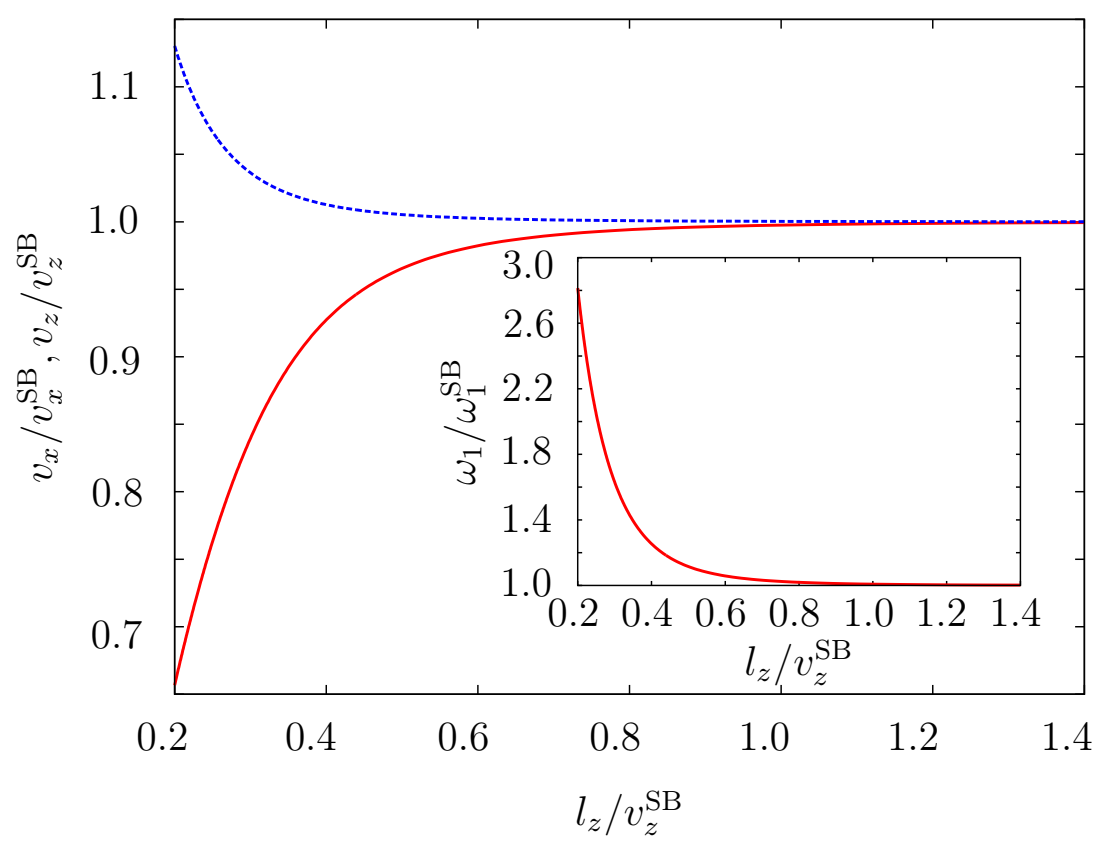

Figure 6.6.: Widths $v_{z}$ (red solid curve) and $v_{x}$ (blue dashed curve) obtained using the variational Gaussian ansatz for a Dy condensate in a spherical trap $(\lambda=1)$ with $N=5000$ atoms, and $a_{s}=80 a_{B}$. The results are normalized to the corresponding widths $v_{z}^{\mathrm{SB}}$ and $v_{x}^{\mathrm{SB}}$ of the SB solution for those parameters, and plotted as a function of the ratio $l_{z} / v_{z}^{\mathrm{SB}}$, with $l_{z}$ the oscillator length of the $z$ confinement. Inset: lowest excitation mode for the same parameters. For these parameters $v_{x}^{\mathrm{SB}}=0.3 \mu \mathrm{m}$ and $v_{z}^{\mathrm{SB}}=3.3 \mu \mathrm{m}$.

\subsection{Excitations}

In this section we study the lowest-lying eigenmodes of BECs stabilized by the LHY corrections. We evaluate them from the condensate response to an abrupt small change of the trap frequencies $\omega_{x, y, z}=(1+\epsilon) \omega_{x, y, z}$, with $\epsilon=0.01$. The subsequent dynamics is evaluated by real-time evolution of Eq. (5.2). We monitor in particular the variances of the BEC along each of the three spatial directions. The Fourier analysis of these variances reveals the underlying frequencies as depicted in Fig. 6.7 for the case of a spherically trapped Dy BEC with $N=20000$ atoms. These results are in very good agreement with the Gaussian results obtained from Eqs. (6.7) and (6.8).

Deep in the mean-field regime $\left(a_{s}=130 a_{B}\right.$ in Fig. 6.7) the lowest excitation is given by a radial quadrupole mode, $(1 / \sqrt{2},-1 / \sqrt{2}, 0)$, where we employ the eigenvector notation introduced in Sec. 6.1. The energetically second lowest mode is a 3D quadrupolar one, $(-0.53,-0.53,0.66)$, and the third mode is a monopole-like one, $(0.5,0.5,1 / \sqrt{2})$. The character of these modes changes when the BEC crossovers into the droplet regime, $a_{s}<90 a_{B}$ in Fig. 6.7. Due to the marked elongation of the droplet along the dipole direction, axial (along $z$ ) and radial (on the xy plane) modes approximately decouple. The 3D quadrupole-like mode becomes the lowest lying one, but it becomes almost completely an axial mode along $z,(-0.05,-0.05,0.99)$. The radial quadrupole mode does not change 


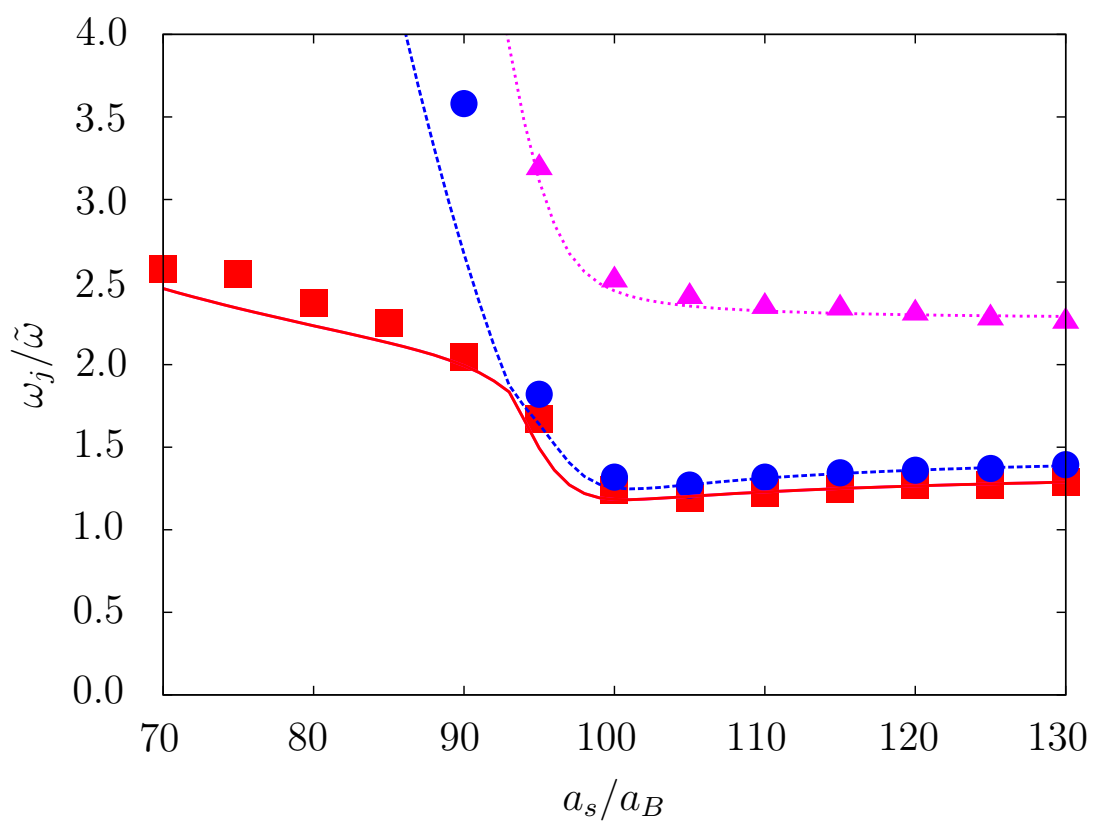

Figure 6.7.: Lowest excitation frequencies of a spherically trapped Dy BEC with $N=20000$ atoms and $\tilde{\omega} / 2 \pi=70 \mathrm{~Hz}$. The numerical results for the three lowest modes are marked in order of growing energy by squares, circles, and triangles. These results are obtained by monitoring the condensate after a slight trapping quench $\left(\epsilon=0.01, \omega_{x, y, z} \rightarrow(1+\right.$ $\left.\epsilon) \omega_{x, y, z}\right)$. The corresponding frequencies evaluated from the Gaussian ansatz are depicted by, respectively, solid, dashed, and dotted lines.

its character, whereas the 3D monopole-like mode becomes approximately a 2D monopole mode $(0.7,0.7,0.07)$. Due to the large aspect ratio of the droplet, the quasi-radial modes become much more energetic than the quasi-axial mode. As a consequence a slight quench of the trap frequencies just excites the lowest mode in the droplet regime.

Due to similar reasons, in a SB BEC the lowest mode remains quasi-axial. Deep in the $\mathrm{SB}$ regime, this mode retains a slight 3D quadrupole character, as for the case of trapped BECs. However, close to the instability the mode becomes 3D monopole-like. The softening of this mode marks the unbinding of the dipolar BEC. Figure 6.8 shows that whereas the lowest eigenenergy of the SB solution grows monotonously with decreasing $a_{s}$ (inset of Fig. 6.8), it does present a maximal value as a function of $N$, decreasing at large $N$. At this maximum the mode changes, for decreasing $N$, from quadrupole to monopole character. Note that the Gaussian ansatz describes well the qualitative dependence of the excitation energy, although the quantitative value may significantly differ due to the clearly non-Gaussian nature of the BEC deep inside the SB regime (see Figs. 6.4(b) and (c)).

Finally, let us note that the frequency of the lowest-lying mode of the trapped droplet departs significantly from that of the SB solution if $l_{z} / v_{z}^{\mathrm{SB}}<1$ (inset of Fig. 6.6). For $N=5000$ Dy atoms in a spherical trap with $\tilde{\omega} / 2 \pi=70 \mathrm{~Hz}$ and $a_{s}=80 a_{B}, \omega_{1} / \omega_{1}^{\mathrm{SB}} \simeq 1.85$ (we recall that for this case, $v_{z}, v_{x}$ and $n_{P}$ presented a relatively small departure from the $\mathrm{SB}$ values). 


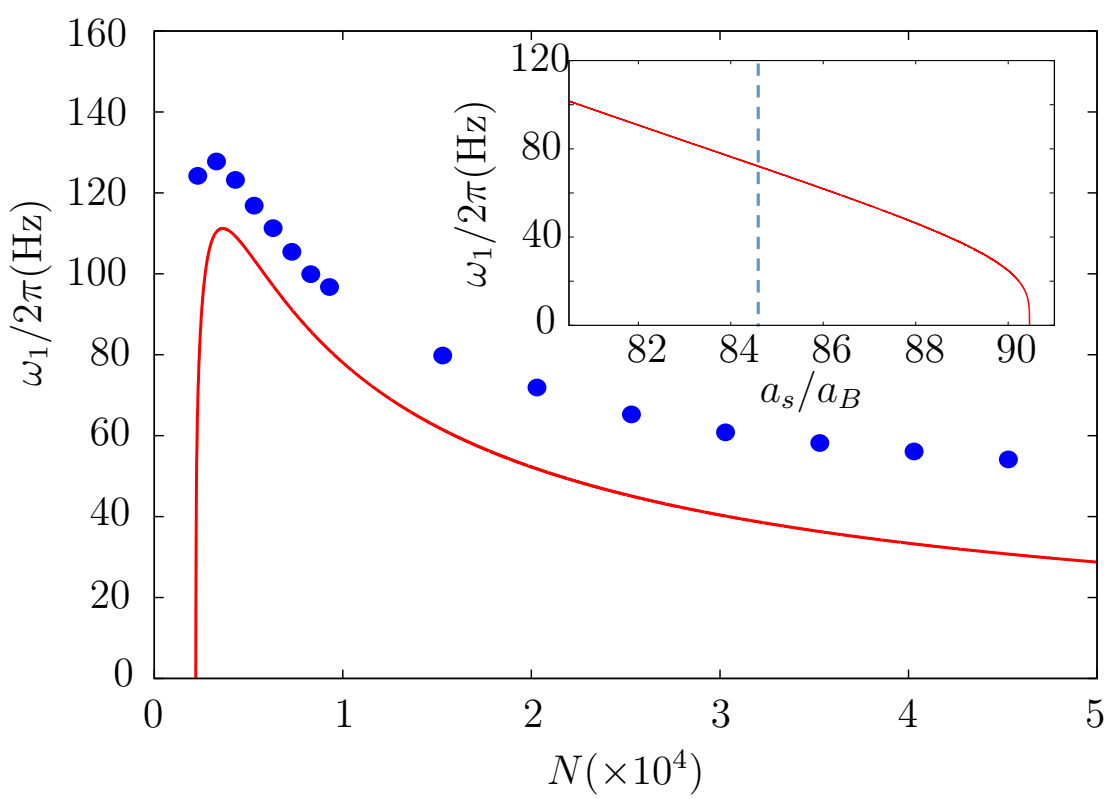

Figure 6.8.: Lowest eigenenergy of the SB solution for a Dy BEC as a function of $N$ for $a_{s}=80 a_{B}$; inset: same as a function of $a_{s}$ for $N=5000$. The dashed lines mark the point at which the lowest mode changes from a quadrupole to a monopole character before becoming unstable. The curves have been obtained using the variational Gaussian ansatz, whereas the circles are obtained directly from the numerical simulation of Eq. (5.2).

\subsection{Conclusions}

In this chapter we have analyzed in detail the ground-state properties and lowestlying excitations of a dipolar BEC in the presence of LHY stabilization extending significantly the initial studies shown in chapter 5 . For a trap aspect ratio $\lambda>\lambda_{c r}$ the BEC presents three marked regions, a mean-field region, in which the LHY term is perturbative, a droplet region, and an intermediate multistable region. The mean-field to multistable boundary is characterized by a first order phase transition, at which the droplet solution becomes the global ground state. For $\lambda<\lambda_{c r}$ there is a crossover between the mean-field solution and the droplet one, marked by a characteristic functional dependence of the peak density and the lowest-lying excitation with the scattering length $a_{s}$. Although we have focused in this chapter on the particular case of dysprosium, similar results characterize other strongly dipolar gases, in particular recent erbium experiments [154], see chapter 7 .

A major consequence of the quantum stabilization is the possibility to create three-dimensionally self-bound condensates, which would be hence characterized by a vanishing time-of-flight expansion velocity. We have shown however that, due to the elongation of the BEC along the dipole direction, the convergence of the trapped solution to the ground state self-bound one demands under typical conditions a rather weak confinement. If the confinement is not weak enough, the properties of the trapped BEC may significantly differ from the ground state self-bound case, especially in what concerns the lowest-lying mode. As a result 
an abrupt switch off of the trap in time-of-flight experiments would create rather an excited solution, which may result in a finite time-of-flight expansion [154].

The existence of self-bound droplets was experimentally proven in very recent dysprosium experiments [78]. There a BEC was created in the mean-field stable regime at large scattering length for a trap aspect ratio $\lambda=1.5$ in order to form a single macro droplet when the scattering length is reduced. A magnetic field gradient was applied in order to levitate the atomic cloud to compensate the gravitational force. Then the trap was switched off and the cloud imaged after a levitation time $t_{\text {lev }}$. The results showed a 3D self-bound droplet which is stable up to $90 \mathrm{~ms}$ after which the atom number becomes smaller than the critical value so that the system gets unbound.

Self-bound condensates could not be observed up to now in erbium experiments for reasons discussed in chapter 7 . 


\section{Quantum fluctuations in an erbium BEC}

In this chapter we present the results of our recent collaboration with erbium experiments at the group of F. Ferlaino in Innsbruck [154]. We show that the LHY stabilization mechanism found in dysprosium is a general effect that plays also a significant role in erbium experiments. We study quantum filaments in the experimentally novel regime of cigar shaped traps in which for small scattering lengths a single macro droplet is created. Moreover, we investigate the properties of the condensate all the way from the mean-field to the droplet regime, focusing on the lowest collective oscillation and the time of flight (TOF) expansion. Additionally, we investigate in detail the role of three-body losses as they are significant for the static and dynamic properties of erbium droplets.

In section 7.1 we introduce the framework of this chapter followed by a discussion of the significance of three-body losses in section 7.2. In section 7.3 we study the axial mode of an erbium condensate and in section 7.4 we investigate the expansion dynamics. We conclude the chapter in section 7.5.

\subsection{Framework}

In order to compare our theoretical results with the experimental measurements we tried to describe the experimental situation as close as possible and therefore adapted our calculations to the experimental procedures.

We apply again a mean-field description and use the non-linear non-local Schrödinger equation derived in section 5.1 including the LHY corrections. We extend this equation by a three-body loss term as explained in section 1.5 which leads to

$$
\begin{aligned}
\mathrm{i} \hbar \frac{\partial \Psi(\mathbf{r}, t)}{\partial t}= & {\left[-\frac{\hbar^{2} \nabla^{2}}{2 M}+V_{\mathrm{tr}}(\mathbf{r})+g n(\mathbf{r}, t)+\int d^{3} r^{\prime} V_{\mathrm{dd}}\left(\mathbf{r}-\mathbf{r}^{\prime}\right) n\left(\mathbf{r}^{\prime}, t\right)\right.} \\
& \left.+\frac{32}{3 \sqrt{\pi}} g n(\mathbf{r}, t) \sqrt{n(\mathbf{r}, t) a_{s}^{3}} F\left(\epsilon_{\mathrm{dd}}\right)-\mathrm{i} \hbar \frac{L_{3}\left(a_{s}\right)}{2} n(\mathbf{r}, t)^{2}\right] \Psi(\mathbf{r}, t) .
\end{aligned}
$$

Due to the weaker dipole-dipole interactions in erbium the scattering length $a_{s}$ close to the mean-field stability threshold is smaller than in the case of dysprosium. Therefore the densities of the formed quantum droplets are higher leading to more significant three-body losses in this system. As the three-body loss rate $L_{3}$ depends on the scattering length we include this experimentally determined dependence $L_{3}\left(a_{s}\right)$ in our description. To this end we use a fit of the experimental data which reads

$$
L_{3}\left(a_{s}\right)=e^{14.8677-0.3734 \cdot\left(a_{s} / a_{B}\right)+0.00217 \cdot\left(a_{s} / a_{B}\right)^{2}} \cdot 10^{-41} \frac{m^{6}}{s} .
$$


The fit gives a good approximation of the loss rate for scattering length values between $45 a_{B}$ and $92 a_{B}$. As three-body losses are a dynamical effect we consider this term only in real-time evolutions and neglect it in discussions of ground state solutions derived by imaginary time evolutions. As discussed in chapter 6, Eq. (7.1) sustains stable ground-state solutions for any value of the scattering length and trapping geometry due to the repulsive LHY term. In contrast to pancake traps $(\lambda>1)$, elongated traps along the dipole direction $(\lambda<1)$ present a unique energy minimum. In the $\epsilon_{\mathrm{dd}}$ parameter space, the corresponding wave function exhibits a smooth crossover from a dilute BEC to a single, highdensity, macro droplet solution for increasing $\epsilon_{\mathrm{dd}}$. In the following, we focus on this case where we consider a three-dimensional harmonic trap with frequencies $\omega_{x, y, z} /(2 \pi)=(155.1,196.3,17.3) \mathrm{Hz}$.

As in chapter 6 a Gaussian ansatz for the condensate wave function provides a good qualitative (and to a large extent quantitative) insight in the physics of dipolar condensates in the presence of LHY stabilization. In contrast to the ansatz of chapter 6 we consider now a more general one including three-body losses of the form

$$
\psi(\mathbf{r}, t)=\sqrt{N(t)} e^{\mathrm{i} \phi(t)} \prod_{\eta=x, y, z} \frac{\mathrm{e}^{-\frac{\eta^{2}}{2 w_{\eta}(t)^{2}}+\mathrm{i} \eta^{2} \beta_{\eta}(t)}}{\pi^{1 / 4} w_{\eta}(t)^{1 / 2}},
$$

where the atom number $N$ is time dependent. The variational parameters are the number of atoms $N(t)$, the global phase $\phi(t)$, the widths $w_{\eta}(t)$, and the phase curvatures $\beta_{\eta}(t)$. The Lagrangian density takes the same form as in section 6.1 reading

$$
\begin{aligned}
\mathcal{L}= & \frac{\mathrm{i} \hbar}{2}\left(\psi \frac{\partial \psi^{*}(\mathbf{r}, t)}{\partial t}-\psi^{*} \frac{\partial \psi(\mathbf{r}, t)}{\partial t}\right)+\frac{\hbar^{2}}{2 m}|\nabla \psi(\mathbf{r}, t)|^{2}+V_{\mathrm{tr}}(\mathbf{r})|\psi(\mathbf{r}, t)|^{2} \\
& +\frac{g}{2}|\psi(\mathbf{r}, t)|^{4}+\frac{2}{5} g_{\mathrm{LHY}}|\psi(\mathbf{r}, t)|^{5}+\frac{1}{2} \int d^{3} r^{\prime} V_{\mathrm{dd}}\left(\mathbf{r}-\mathbf{r}^{\prime}\right)|\psi(\mathbf{r}, t)|^{2}\left|\psi\left(\mathbf{r}^{\prime}, t\right)\right|^{2}
\end{aligned}
$$

Inserting the ansatz (7.3) into Eq. (7.4) and integrating over position space leads to $L=\int d^{3} r \mathcal{L}$ :

$$
\begin{aligned}
L & =N\left\{\hbar \dot{\phi}+\frac{\hbar}{2} \sum_{\eta} \dot{\beta}_{\eta} w_{\eta}^{2}+\frac{M}{4} \sum_{\eta} \omega_{\eta}^{2} w_{\eta}^{2}+2 \frac{\hbar^{2}}{2 M} \sum_{\eta}\left(\beta_{\eta}^{2} w_{\eta}^{2}+\frac{1}{4 w_{\eta}^{2}}\right)\right\} \\
& +N^{2}\left\{\frac{g\left(1+\epsilon_{d d} F\left(w_{z} / w_{x}, w_{z} / w_{y}\right)\right)}{2(2 \pi)^{3 / 2} \prod_{\eta} w_{\eta}}\right\}+N^{5 / 2}\left\{\left(\frac{2}{5}\right)^{5 / 2} \frac{g_{L H Y}}{\pi^{9 / 4} \prod_{\eta} w_{\eta}^{3 / 2}}\right\},
\end{aligned}
$$

with $F\left(w_{z} / w_{x}, w_{z} / w_{y}\right)$ defined in Eq. (6.6). The variational parameters are determined from the corresponding Euler-Lagrange equations which include explicitly the three-body losses on the right hand side [155]:

$$
\frac{d}{d t}\left(\frac{\partial L}{\partial \dot{\lambda}}\right)-\frac{\partial L}{\partial \lambda}=\int d^{3} r\left[\Gamma(\mathbf{r}) \frac{\partial \psi^{*}(\mathbf{r})}{\partial \lambda}+\Gamma^{*}(\mathbf{r}) \frac{\partial \psi(\mathbf{r})}{\partial \lambda}\right]
$$

where $\lambda=N, \phi, w_{\eta}, \beta_{\eta}$, and $\Gamma(\mathbf{r})=-\mathrm{i} \hbar \frac{L_{3}}{2}|\psi(\mathbf{r})|^{4} \psi(\mathbf{r})$. Introducing the dimensionless units $\tau=\tilde{\omega} t, w_{\eta}=\tilde{l} v_{\eta}, \tilde{l}=\sqrt{\hbar / M \tilde{\omega}}$, with $\tilde{\omega}=\left(\prod_{\eta} \omega_{\eta}\right)^{1 / 3}$, and after 

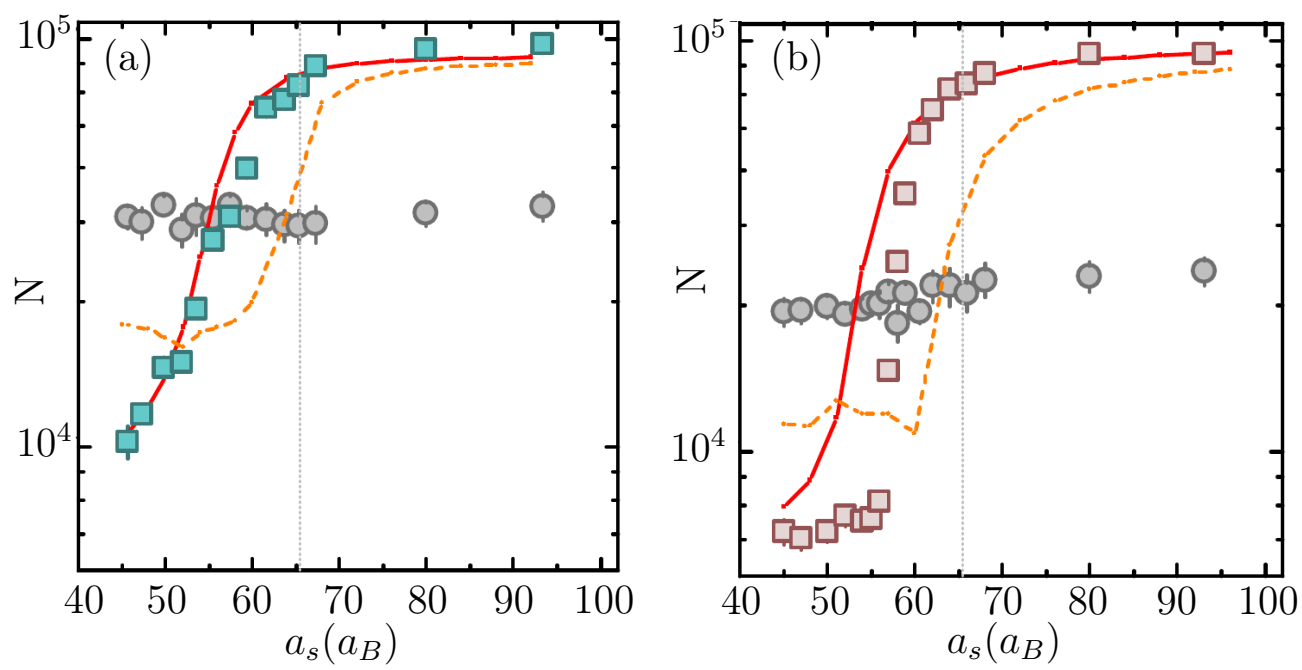

Figure 7.1.: Number of atoms remaining in the condensate after (a) a nonadiabatic $\left(t_{\mathrm{r}}=10 \mathrm{~ms}, t_{\mathrm{h}}=8 \mathrm{~ms}\right)$ and $(\mathrm{b})$ an adiabatic $\left(t_{\mathrm{r}}=45 \mathrm{~ms}\right.$, $t_{\mathrm{h}}=0 \mathrm{~ms}$ ) ramp. The blue squares are the experimental results for the condensate and the gray circles for the thermal component. The data shows a better agreement with the theory with the LHY term (red solid line) as compared to without the LHY term (orange dashed line).

some algebra, we obtain a close set of equations for the Gaussian widths and the number of atoms:

$$
\begin{gathered}
\dot{N}=-\frac{3 R}{\prod_{\eta} v_{\eta}^{2}} N^{3} \\
\ddot{v}_{\eta}=-v_{\eta}\left[\frac{7 R^{2} N^{4}}{\prod_{\eta^{\prime}} v_{\eta^{\prime}}^{4}}+\frac{2 R N^{2}}{\prod_{\eta^{\prime}} v_{\eta^{\prime}}^{2}} \sum_{\eta^{\prime \prime} \neq \eta} \frac{\dot{v}_{\eta^{\prime \prime}}}{v_{\eta^{\prime \prime}}}\right]-\frac{\partial U}{\partial v_{\eta}},
\end{gathered}
$$

with $R=\frac{L_{3}}{\pi^{3} 3^{5 / 2} \tilde{\omega} \tilde{l}^{6}}$, and the potential $U$ is defined in Eq. (6.5).

Due to its simplicity, Eqs. (7.7) and (7.8) permit a much more flexible simulation of the exact experimental conditions and sequences compared to the obviously more exact but numerically much more cumbersome simulation of Eq. (7.1). We note that the results obtained from this generalized Gaussian ansatz are qualitatively and to a large extend quantitatively in very good agreement with the results obtained from Eq. (7.1), as can be seen in the next sections.

\subsection{Loss dynamics}

We study first the time evolution of the atom number $N$ across the BEC-todroplet crossover in order to investigate the respective roles of three-body losses and quantum fluctuations. Since in the droplet regime the density $n(\mathbf{r})$ increases dramatically, three-body losses are expected to play an important role even for moderate and low values of $L_{3}$.

At first we calculate the ground state of an erbium condensate initially prepared with $N=1.2 \times 10^{5}$ atoms at a scattering length $a_{s}=67 a_{B}$. Then we ramp down the scattering length linearly during a fixed ramping time $t_{r}$ to a final scattering 


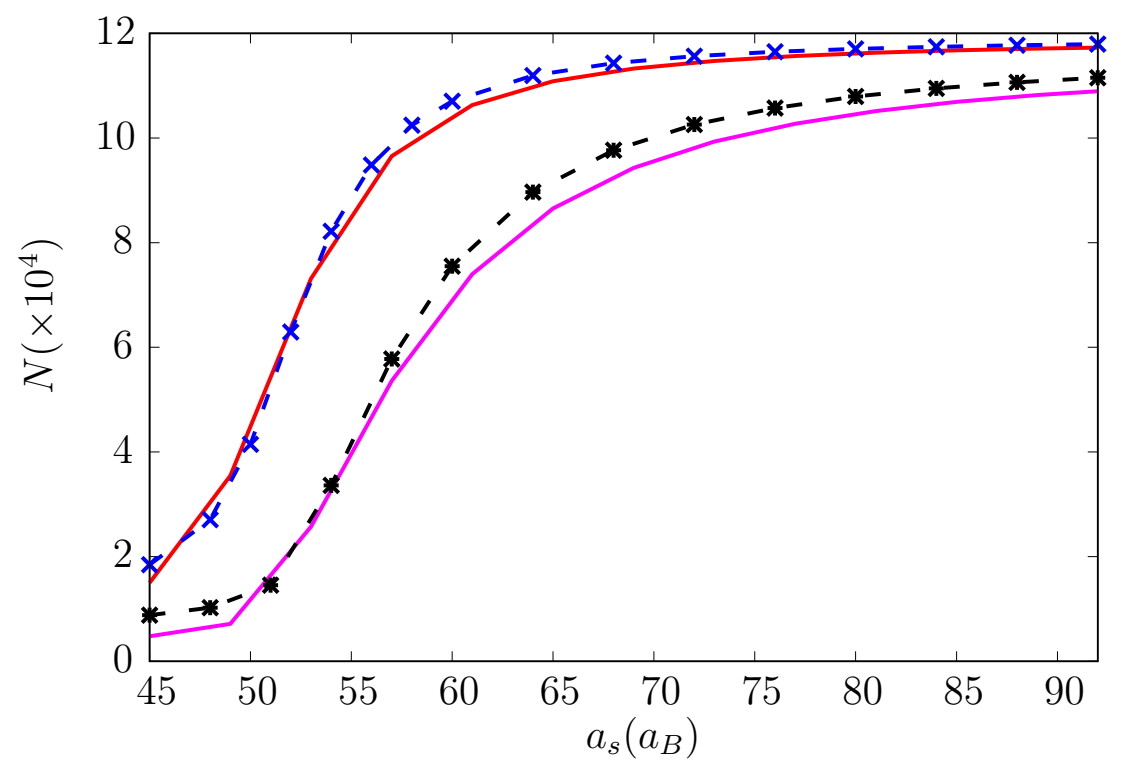

Figure 7.2.: Comparison of the loss-curves for an erbium condensate with LHY corrections calculated by Eq. (7.1) (dashed lines) and the Gaussian ansatz (solid lines). The calculations are done for a $10 \mathrm{~ms}$ ramp (blue and red curve) and for a ramp of $45 \mathrm{~ms}$ (black and purple curves). The results are in very good agreement, validating the use of the Gaussian ansatz.

length and hold the condensate afterwards for the fixed time $t_{h}$ (this sequence is basically that employed in the erbium experiments). During the holding time we let the system evolve without any perturbations as we hold all system parameters constant.

Figure 7.1 (a-b) summarizes the experimental and theoretical results after a non-adiabatic ramp of the scattering length $\left((\mathrm{a}), t_{r}=10 \mathrm{~ms}, t_{h}=0 \mathrm{~ms}\right)$ and an adiabatic ramp ((b), $\left.t_{r}=45 \mathrm{~ms}, t_{h}=0 \mathrm{~ms}\right)$. The $x$-axis shows the final scattering length after the ramp and the $y$-axis the total number of atoms remaining in the condensate after the holding time. The adiabatic and the non-adiabatic case show a similar behavior, i.e. the atom number is almost constant for scattering lengths $a_{s}>a_{\mathrm{dd}}$ and shows a sharp drop starting around $a_{s} \sim a_{\mathrm{dd}}\left(a_{\mathrm{dd}}\right.$ is indicated in Fig. (7.1) as a dotted vertical line at $66 a_{B}$ ). The atom number in the case of the adiabatic ramp is always smaller than in the non-adiabatic one due to the longer time at which the system looses atoms. The observed evolution of $N$ is very well reproduced by our theoretical calculations obtained from a real-time evolution of Eq. (7.1).

In order to make the effects of quantum fluctuations clear we performed also calculations without LHY corrections which can be seen in Fig. (7.1) as orange dashed lines. These calculations predict much larger losses as can be seen in the experiment. For small scattering lengths a condensate without LHY corrections collapses leading to a dramatic drop in the atom number and the formation of a fuzzy cloud with many excitations and small density. Therefore three-body losses in the cloud after the collapse are small and the atom number does not drop below 20000 atoms in stark contrast to the experiment and the calculations 

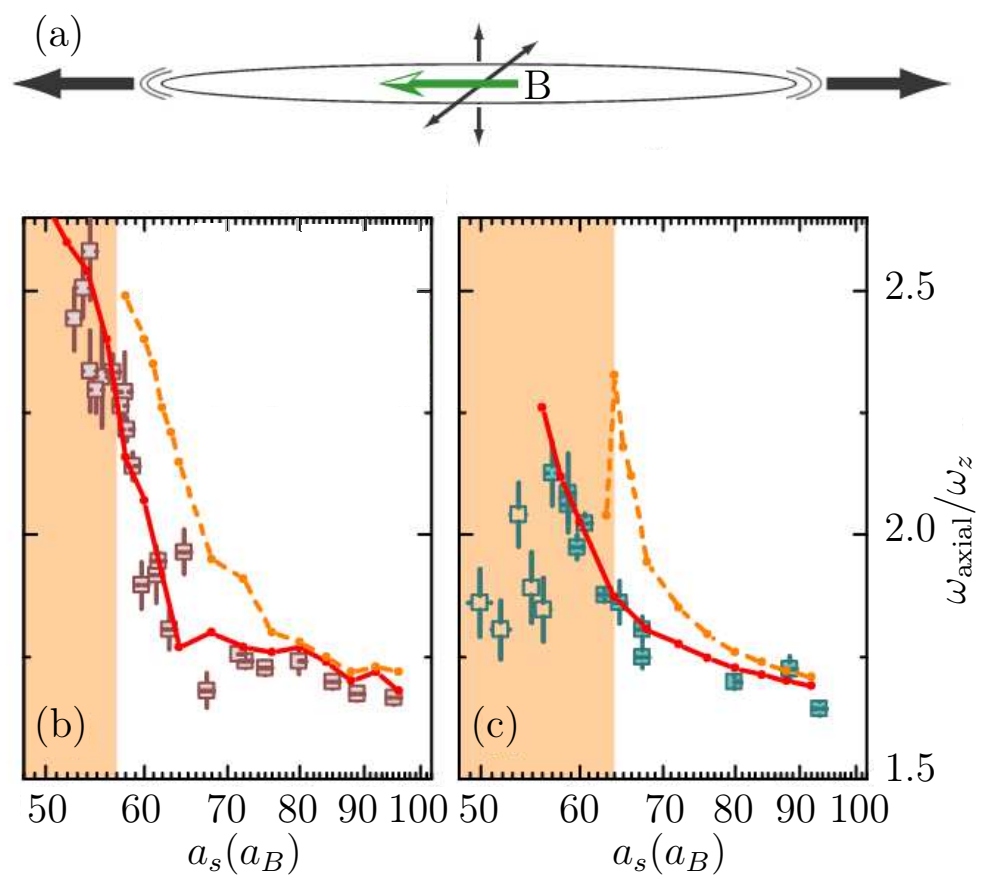

Figure 7.3.: Axial frequency of an erbium condensate after a ramp to a fixed scattering length $a_{s}$. (a) illustrates the axial mode deep in the droplet regime with the black arrows sketching the oscillations of the widths of the gas where the size indicates their relative amplitudes. (b-c) shows the measured frequency $\omega_{\text {axial }}$ in units of $\omega_{z}$ (blue squares) as a function of the final scattering length $a_{s}$ after a ramping time of $t_{\mathrm{r}}=100 \mathrm{~ms}(\mathrm{~b})$ and $t_{\mathrm{r}}=10 \mathrm{~ms}$ (c). The theoretical curves show results with (red solid line) and without (orange dashed line) LHY corrections.

with LHY correction.

The behavior in the thermal cloud which could be measured in the experiment is worth mentioning. The number of atoms in the thermal component $N_{\mathrm{t}}$ remains mainly unaltered over the whole range of scattering lengths and the whole system does not show any appreciable heating. This suggests that the condensed atoms, which are ejected from the core, leave the trap instead of being transferred to the thermal component, confirming a picture in which the thermal and the condensed component have uncoupled dynamics.

The theoretical predictions for the non-adiabatic ramp match better with the experiment as for the adiabatic one. One reason is that quantum fluctuations modify also the three-body losses [156], i.e. lead to an enhanced loss rate, which we have not taken into account in our theoretical simulations. Therefore, the larger predicted atom numbers in our simulation is based on the simple noninteracting value of $L_{3}$ compared to the experiment. We estimate that the change of the three-body loss rate due to quantum fluctuations is about $30 \%$ for the typical parameters in the experiment. Additionally, the local density approximation and the validity of the LHY term become a worse description of the system for small scattering lengths. 


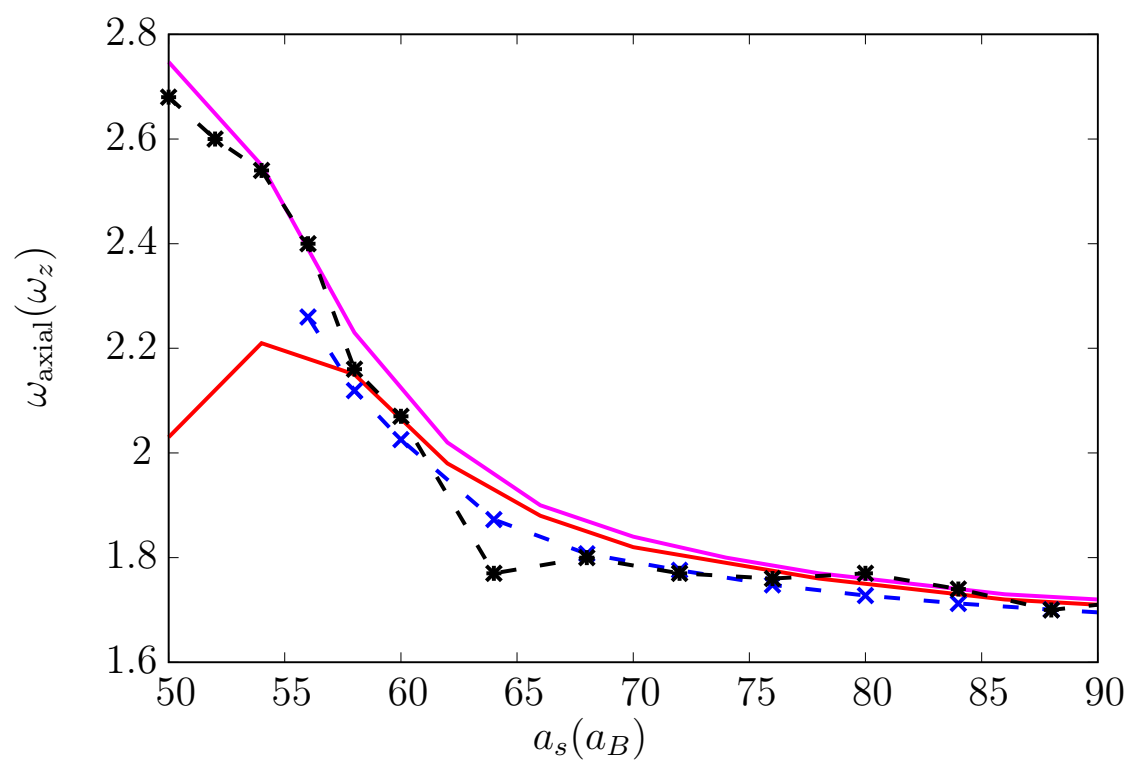

Figure 7.4.: Comparison of the axial frequencies calculated by a real time evolution of Eq. (7.1) (dashed lines) and the Gaussian ansatz (solid lines). The calculations are done for a $10 \mathrm{~ms}$ ramp (blue and red curve) and for a ramp of $100 \mathrm{~ms}$ (black and purple curves).

\subsection{Collective axial excitation}

In this section we study the lowest collective excitation for different scattering lengths all the way from the mean-field to the droplet regime. We focus on this mode as this is the only one which can be excited in an easy way in the experiment for arbitrary scattering lengths. It has an almost pure character of an axial excitation deep in the droplet regime with only a very small amplitude perpendicular to the dipole direction (see Fig. 7.3a). Again we precisely account for the experimental sequence by performing real-time evolutions starting from the ground state of Eq. (7.1) at $a_{s}=67 a_{B}$ with $N=1.2 \times 10^{5}$ atoms. We simulate a linear ramp in the scattering length from $67 a_{B}$ to a variable final value in the ramping time $t_{\mathrm{r}}$, followed by a compression of the axial trap from $\omega_{\mathrm{z}}=17.3 \mathrm{~Hz}$ to $21 \mathrm{~Hz}$ during $8 \mathrm{~ms}$ and afterwards change back the frequency instantaneously to $\omega_{z}=17.3 \mathrm{~Hz}$. This excites the condensate in a clear way along the $z$-axis. Then, we hold the condensate in the trap, let the system evolve and record the axial width (the standard deviation of the density distribution $n(\mathbf{r}), \sigma_{z}=\sqrt{\left\langle z^{2}\right\rangle}$ ) as a function of the subsequent holding time $t_{h}$. The evolution of $\sigma_{z}$ is well fitted by a sinusoidal function, whose frequency constitutes our theoretical prediction of $\omega_{\text {axial }}$. Note that with this scheme the number of atoms in the condensate is different for different scattering lengths as explained in the last section.

The results can be seen in Fig. (7.3) where we present the experimental and theoretical results for an adiabatic ramp of $100 \mathrm{~ms}$ in Fig. (7.3b) and for a nonadiabatic ramp of $10 \mathrm{~ms}$ in Fig. (7.3c). We normalize the frequencies of the axial mode to the frequency of the trap in the axial direction $\omega_{z}$. We performed again simulations with (red solid lines) and without LHY term (orange dashed lines). The theoretical results without LHY term fail for $a_{s} \leq a_{c}$ (orange area) due 


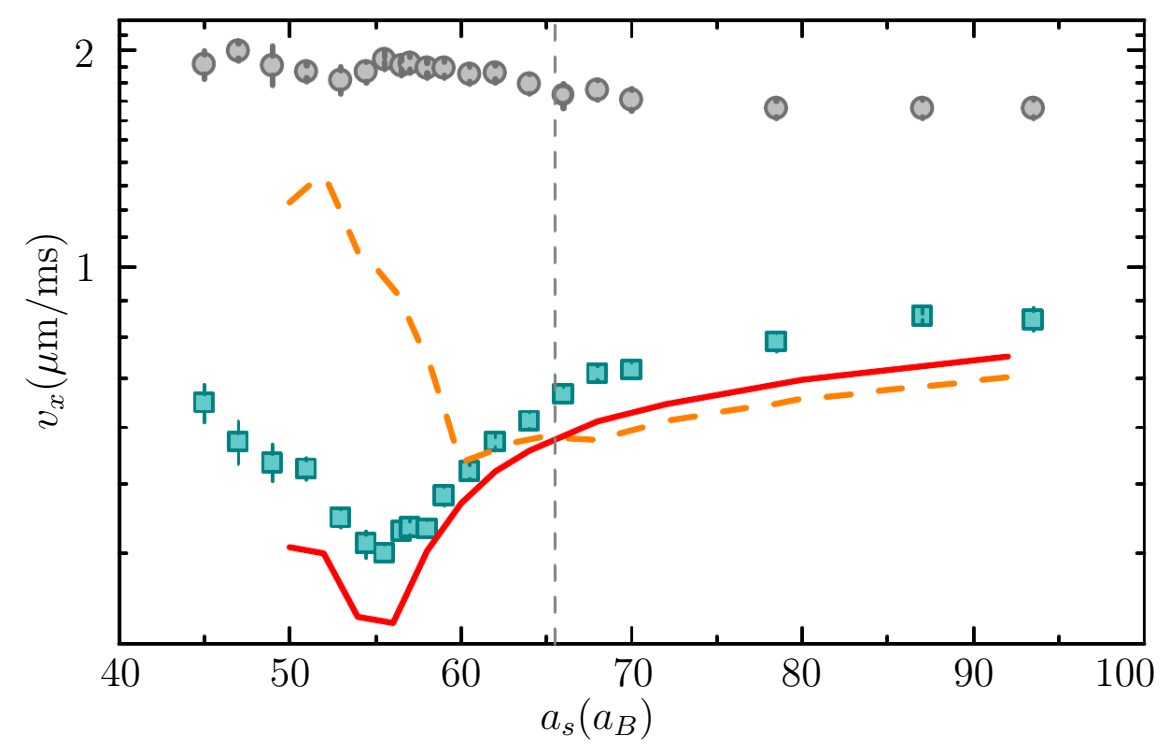

Figure 7.5.: $v_{x}$ as a function of $a_{s}$. The experimental data (blue squares) is in good agreement with our theoretical predictions including the LHY term (red solid line) and rule out a purely mean-field scenario (orange dotted line). For clarity, we only show $v_{x}$. Similar results are found for $v_{z}$ as it is shown in Fig. (7.7). For comparison, the $a_{s^{-}}$ independent expansion velocities of the thermal component are also shown (circles).

to the occurrence of collapsing dynamics which rules out the collective mode picture. Note that this collapsing behavior is in qualitative disagreement with the experimental observations. Moreover, for decreasing $a_{s} \geq a_{\mathrm{c}}$, the non-LHY curve of $\omega_{\text {axial }}$ is sizably shifted compared to the measurements. In contrast, the experimental results show an excellent match with the theory when the LHY term is included, thus ruling out a pure mean-field scenario and demonstrating the crucial role played by the quantum fluctuations in stabilizing the system. Then, the LHY corrections qualitative modify the phase diagram and drive the formation of a special coherent state, namely a single macro-droplet. Note that the value for $a_{c}$ differs for adiabatic and non-adiabatic ramps as the different ramp and holding times result in a different numbers of atoms in the condensate when measuring the frequency. This shifts the boundary of mean-field instability for large $t_{r}$ to smaller scattering lengths $\left(a_{c}=57 a_{B}\right.$ for a $100 \mathrm{~ms}$ ramp and $a_{c}=64 a_{B}$ for a $10 \mathrm{~ms}$ ramp).

Moreover the condensate looses atoms during the holding time which shifts the frequencies during their measurement. For large scattering lengths this effect is too small to be observed in either the experiment or the theoretical calculations. However for the non-adiabatic ramp the frequency shift becomes very large for $a_{s}<56 a_{B}$. Additionally the condensate becomes highly excited getting far away from the linear perturbation regime so that no reasonable frequency can be extracted anymore. Therefore the theoretical curve with LHY term in Fig. (7.4) end at $a_{s}=56 a_{B}$. 


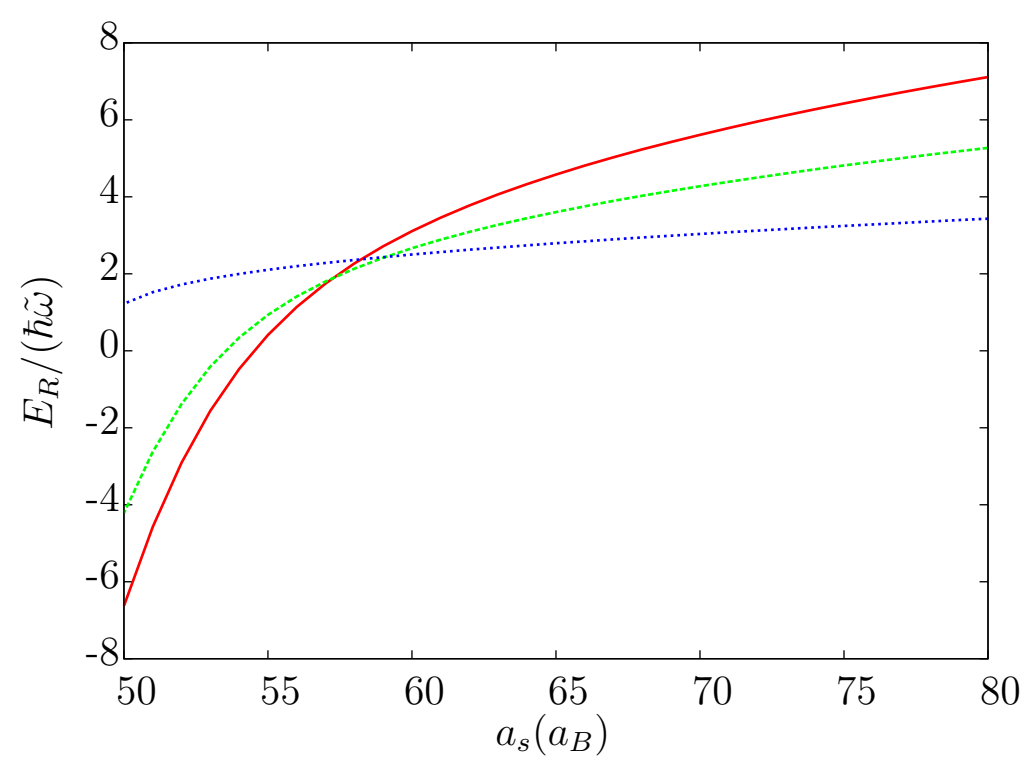

Figure 7.6.: Released energy $E_{R}$ as a function of $a_{s}$ for $N=1.2 \times 10^{5}$ (solid), $5 \times 10^{4}$ (dashed), and $1 \times 10^{4}$ (dotted). Note that $E_{R}<0$ indicates a SB solution.

Additionally we studied the axial frequency within the Gaussian ansatz and the results can be seen in Fig. (7.4). Both calculations match perfectly which is especially remarkable as the macro droplet has a profile that significantly departs from a Gaussian shape.

\subsection{Expansion dynamics}

We turn now our attention to the study of the expansion dynamics of the erbium condensate at different final scattering lengths. The procedure focuses on short timescales with $t_{\mathrm{r}}=10 \mathrm{~ms}$ and $t_{\mathrm{h}}=5 \mathrm{~ms}$ to preserve the high density and large particle number of the BEC. First the ground state of a condensate with $N=1.2 \times 10^{5}$ at $a_{s}=67 a_{B}$ is calculated. Then, the respective ramping and holding dynamics are performed via real-time evolutions and afterwards the trap is switched off abruptly letting the gas expand for a fixed time $t_{\mathrm{TOF}}=25 \mathrm{~ms}$.

As the size of the condensate increases dramatically during the $25 \mathrm{~ms}$ expansion time we apply a multigrid analysis thereby adapting the grid several times during the TOF expansion. This is done in the following way. We calculate the real time evolution in a given grid until the expanding cloud comes close to the boundary of our numerical box. Then we increase the box size either by increasing the spatial step size or by increasing the number of grid points. As we always increase the spatial step size or hold it constant the determination of the new grid points is easy as we simply omit data points, but do not need to interpolate between them. For condensates at large scattering lengths an increase in the step size is always justified as the condensate just smoothly expands. However, for small scattering lengths a lot of small scale excitations and a slowly expanding core structure form in the system so that the reduction of the spatial resolution leads to wrong results of the TOF dynamics. In this case we have to increase the number of grid points. 


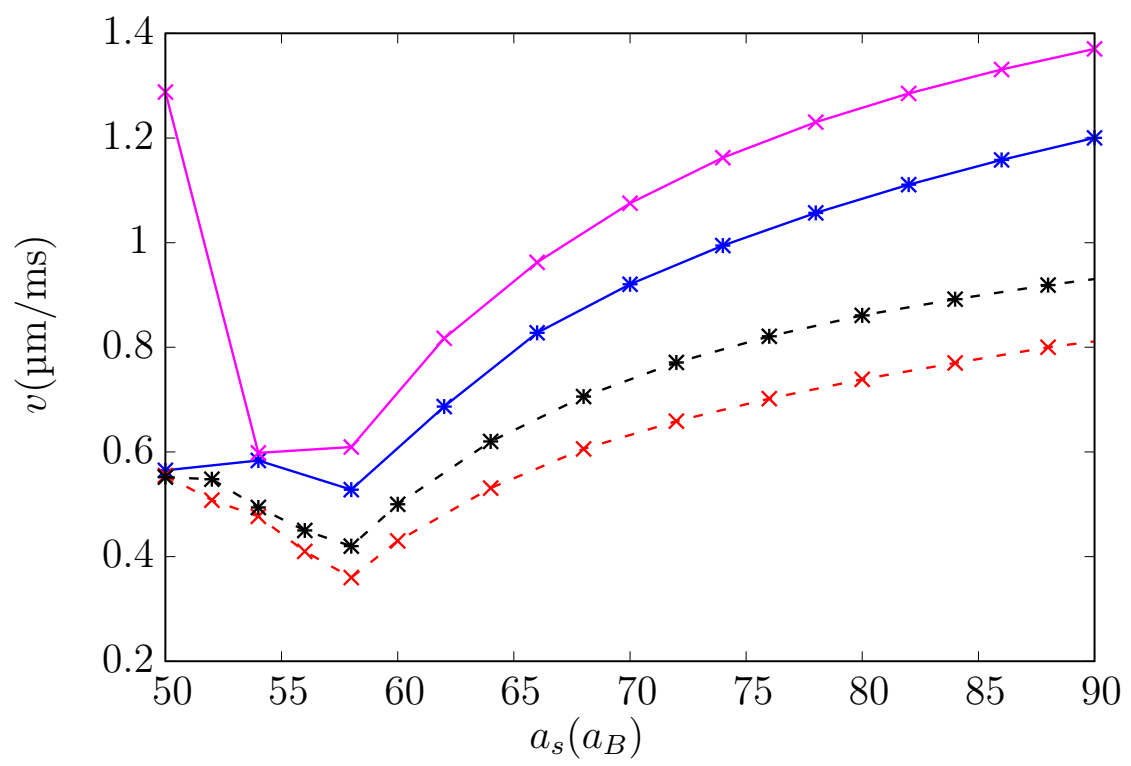

Figure 7.7.: Expansion velocities of an erbium condensate at different scattering lengths after a ramping time of $t_{r}=10 \mathrm{~ms}$ and a holding time of $t_{h}=5 \mathrm{~ms}$. We compare the Gaussian ansatz (solid lines) with the solution from Eq. (7.1) (dashed lines). The red and blue curves show the $x$-velocity and the black and purple curves the $y$-velocity.

As the expansion is anisotropic we have to rescale the grid parameters for every direction independently.

After switching off the trap the cloud first expands slowly in the $x$ - and $y$ direction and then accelerates until a constant expansion velocity within $t_{\mathrm{TOF}} \sim$ $20 \mathrm{~ms}$ is reached. We determine for every time step the size of the condensate via $\sigma_{\eta}=\left(\int d^{3} r \eta^{2} n(\mathbf{r})\right)^{1 / 2}$ with $\eta=x, y, z$ and calculate its derivative numerically in order to extract the expansion velocity. The results can be seen in Fig. (7.5) where we present $v_{x}$ measured in the experiment (blue square) and the theoretical predictions with (red solid line) and without LHY correction (dashed orange line). We conclude that our calculations including the LHY term agree well with the experimental observations whereas the results without LHY term fail for small scattering lengths, i.e. in the droplet regime. The experimental and theoretical curves show a decrease of the expansion velocity with the scattering lengths as the repulsive interactions are decreased. The expansion velocity reaches a minimum at $v_{x} \sim 0.32 \mu \mathrm{m} / \mathrm{ms}$ at $a_{s}=56 a_{B}$ and increases for very small scattering lengths again.

The expansion behavior can be qualitatively well understood considering the released $E_{\mathrm{R}}$ which is the energy of the system when subtracting the energy related with the confinement. In the mean-field scenario, $E_{\mathrm{R}}>0$ as long as the ground state is stable. The BEC expands ballistically and $v_{\eta}$ decreases for decreasing $a_{s}$ and $N$. In the unstable regime, the expansion velocity depends crucially on the value of $t_{\mathrm{h}}$ at which the trap is switched off due to the occurrence of an in-situ collapse dynamics. On the contrary, in the presence of quantum fluctuations, a stable ground state always exists. Assuming a fixed $N$ (i.e. no three-body losses), $E_{\mathrm{R}}$ decreases with decreasing $a_{s}$ and eventually reaches $E_{\mathrm{R}}<0$ for $a_{s}<a_{\mathrm{SB}}$ (see 
(a)

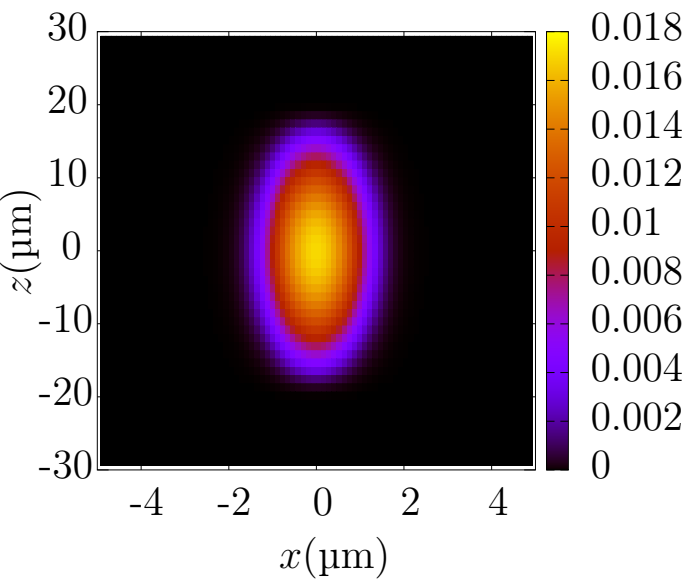

(c)

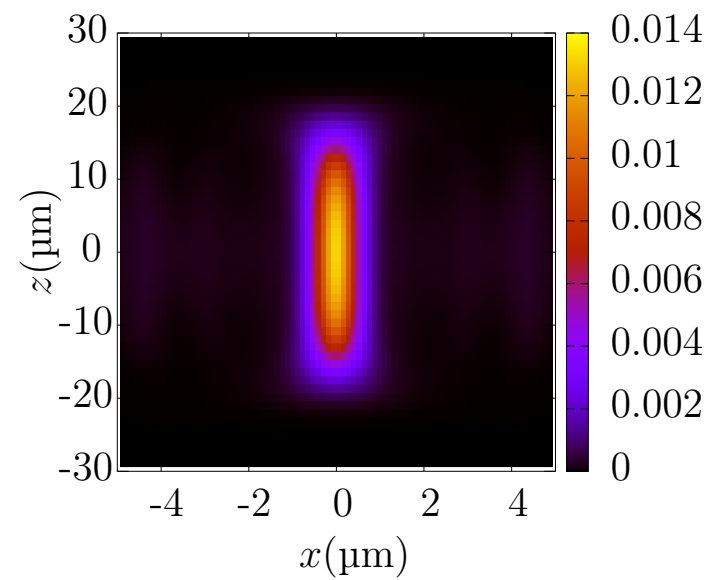

(b)

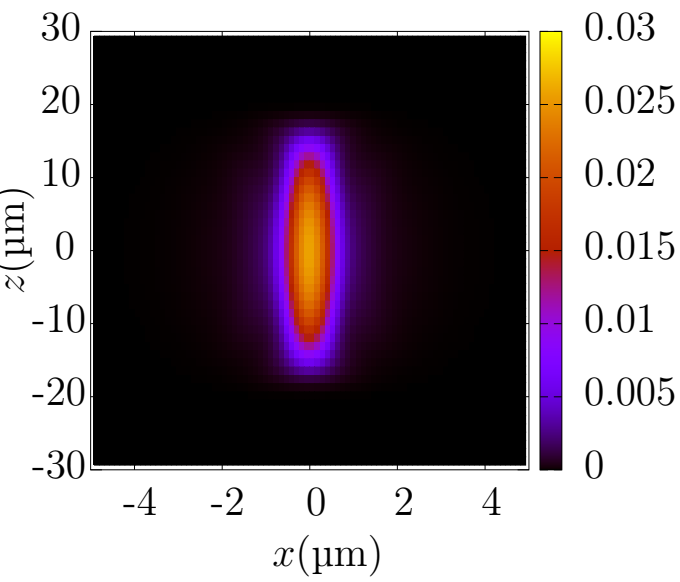

(d)

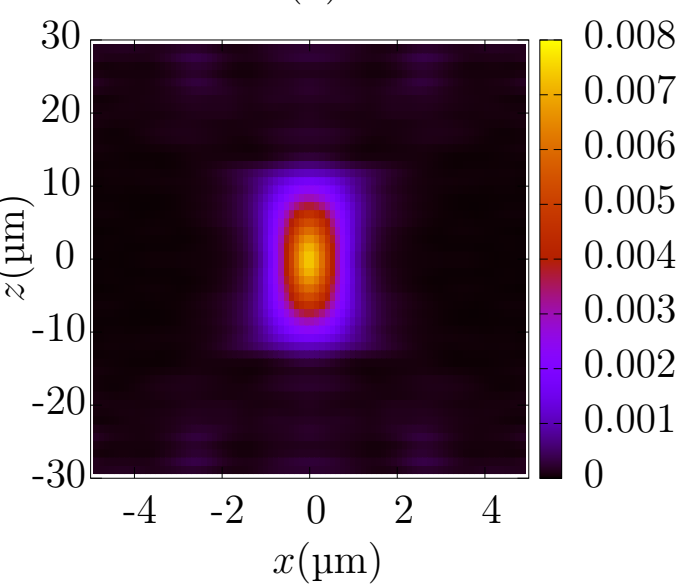

Figure 7.8.: Integrated density profiles $n_{X Z}(x, z) / N$ with $n_{X Z}=\int d y n(\mathbf{r})$ of an erbium condensate initially formed with $N=1.2 \times 10^{5}$ atoms at a scattering length $a_{s}=66 a_{B}$ (a). The scattering length was then instantly ramped down to $a_{s}=54 a_{B}$ and the trap switched off. The pictures show the density profiles at $t=1.5 \mathrm{~ms}(\mathrm{~b}), t=10 \mathrm{~ms}(\mathrm{c})$, and $t=75 \mathrm{~ms}(\mathrm{~d})$.

Fig. (7.6)), marking the onset of the self-bound solution (e.g. $a_{\mathrm{SB}}=56 a_{B}$ for $N=1.2 \times 10^{5}$ ). However, in stark contrast to the mean-field case, $E_{\mathrm{R}}$ increases with decreasing $N$ in the droplet regime. We note that $a_{\mathrm{SB}}$ is then shifted to lower values when $N$ gets reduced by three-body losses, thus affecting the lifetime of the self-bound solution.

The existence of a minimal expansion velocity is thus a direct consequence of the competition between the decrease of $E_{\mathrm{R}}$ for decreasing $a_{s}$ at a fixed $N$, and the increase of $E_{\mathrm{R}}$ for decreasing $N$ in the droplet regime. In the crossover regime, the system smoothly evolves towards a fully self-bound state $\left(v_{\eta}=0\right)$ until three-body losses, occurring in trap or in the initial phase of the expansion, set in to unbind the system and to reduce the lifetime of the droplet state.

The observed slight overall shift of the experimental data compared to the theoretical predictions may be attributed to experimental artifacts coming from (i) the restricted range of $t_{\mathrm{TOF}}$ experimentally accessible, (ii) artifacts of the expansion fit arising in particular from the interplay of the two fit parameters, the empirical expression of the fit or the finite range of expansion time (cf. (i)), 


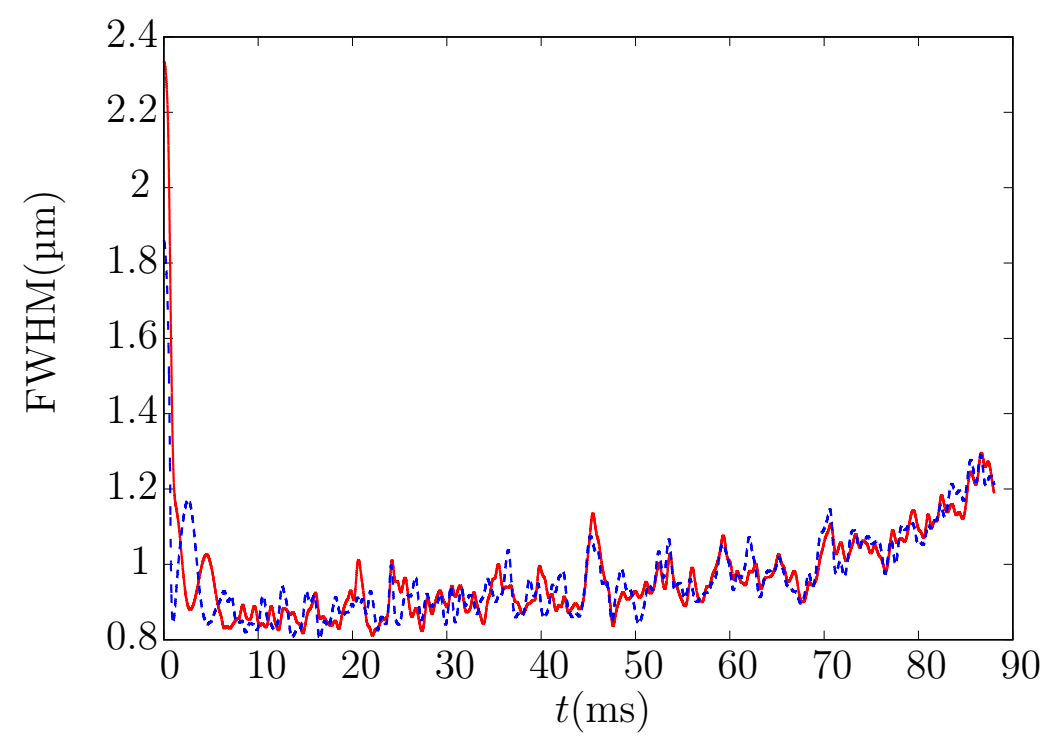

Figure 7.9.: Full width half maximum (FWHM) of an erbium condensate initially formed with $N=1.2 \times 10^{5}$ atoms at a scattering length $a_{s}=66 a_{B}$ in $x$-direction (red solid line) and $y$-direction (blue dashed line). The scattering length was then instantly ramped down to $a_{s}=54 a_{B}$ and the trap switched off.

(iii) artifacts coming from the empirical double Gaussian fit used here to extract $\sigma$. This may lead to misassessment of the size of the coherent part and of its evolution with $t_{\mathrm{TOF}}$.

Fig. (7.7) shows the calculated expansion velocity by means of the Gaussian ansatz and for comparison also the solutions from Eq. (7.1). The Gaussian ansatz shows the same qualitative behavior with a minimum of the expansion velocity at $a_{s}=56 a_{B}$ and even a large increase for small scattering length. However, compared to the full numerical calculations the curves calculated from the Gaussian ansatz are shifted upwards.

\subsection{Conclusion}

In summary, we have demonstrated in the context of erbium experiments the existence of the crossover from a dilute BEC to a quantum droplet state driven by quantum fluctuations. The experiment not only demonstrates that LHY stabilization is a general feature of strongly dipolar gases, but also thoroughly investigates the driving role of quantum fluctuations in dictating the system properties, in particular its atom losses, its collective mode and expansion dynamics. This clear and quantitative demonstration of the impact of quantum fluctuations in dipolar gases intrinsically relies on the unique and precise knowledge of the scattering length in the experiment as that alone enables a direct comparison to a parameter-free theory, which is based on a non-linear non-local Schrödinger equation with LHY correction providing a validation of our theory.

As a final note, we would like to point out that although the experiments in Innsbruck could not observe actual self-bound solutions, it is indeed possible to observe erbium clouds that do not expand in time of flight. In very recent cal- 
culations we considered a condensate with initially $N=1.2 \times 10^{5}$ atoms formed at $a_{s}=66 a_{B}$. Then we applied an instant quench of the scattering length to $a_{s}=54 a_{B}$ and switched off the trap, i.e. $t_{r}=0 \mathrm{~ms}$ and $t_{h}=0 \mathrm{~ms}$. Our numerical results show that the condensate contracts and forms a self-bound droplet with a lifetime on the order of $100 \mathrm{~ms}$ which should be observable in the erbium experiments in Innsbruck. Fig. (7.8) depicts the integrated density profile of the condensate at different times after the ramp and show clearly that the cloud remains self-bound over $75 \mathrm{~ms}$ albeit changing its form due to large particle losses. Fig. (7.9) shows the full width half maximum of the cloud in $x$ and $y$-direction as a function of the time after the ramp. It can be clearly seen that the condensate contracts in a few ms and remains self-bound. The fluctuations show that the self-bound droplet is formed in an excited state. 


\section{Conclusions and Outlook}

In this thesis we showed that quantum fluctuations can arrest the dipole-driven collapse in strongly dipolar systems leading to the creation of stable quantum filaments.

We derived a non-linear non-local Schrödinger equation including a term characterizing quantum fluctuations within the framework of the local density approximation. We showed that in contrast to the mean-field theory a stable ground state solution exists for any scattering length, trapping geometry and particle number in the validity regime of the theory. We find mean-field stable solutions for large scattering lengths and for small scattering lengths a droplet regime in which the LHY term is crucial for the stability of the condensate. The density profile of droplets is always cigar shaped, e.g. elongated along the dipole-direction, irrespective of the underlying trapping geometry and their internal energy decreases to negative values for large particle numbers. For trap aspect ratios $\lambda<1.8$ the two solutions are connected by a smooth crossover in which the density increases dramatically following a specific functional form unique to the LHY stabilization. For larger trap aspect ratios $\lambda>1.8$ a region of multistability can be found following a first order phase transition in which the droplet solution and the metastable mean-field solution coexist.

We showed by incorporating the LHY correction that we can deduce the experimental results of recent dysprosium experiments in which a crystal-like droplet structure is created. Our theoretical investigations showed that the largely negative internal energy of large droplets together with the quasi energy conservation in the experiment leads to the formation of the crystal-like structure. This explains both the long lifetime of the droplets as well as the statistics of the number of droplets and the number of atoms in the droplets.

We investigated the properties of self-bound quantum filaments by mapping their boundary of stability as well as their density profiles which deviate largely from that of trapped condensates. In addition we showed that due to the large size of truly 3D self-bound droplets it is experimentally challenging to create them as the droplets become excited when the trap is switched off. We also investigated the lowest-lying excitation in the self-bound regime showing that the softening of this mode causes instability into evaporation. This mode is almost completely axial regardless of the scattering length and the number of particles and changes its character from a 3D quadrupolar one deep in the droplet regime to a monopole-like one when softening. A more careful study of the excitation modes of a trapped condensate showed that the energy of the lowest excitations increases largely when entering the droplet regime while at the same time the radial and axial modes decouple.

Moreover, we showed the crucial role of LHY corrections in recent erbium experiments in a cigar-shaped trapping geometry. This shows that these stabilization mechanism is a general effect relevant also for other dipolar systems. Furthermore 


\section{Conclusions and Outlook}

these experiments investigated a new regime, i.e. a cigar-shaped trapping geometry in which no phase transition is crossed when entering the droplet regime. Moreover, three-body losses are crucial for the dynamics of these erbium droplets affecting especially the time-of-flight velocity and preventing the observation of self-bound droplets. The experimental and theoretical results are in very good agreement demonstrating both the significance of quantum fluctuations in erbium experiments as well as the applicability of the non-linear non-local Schrödinger equation.

Additionally, we studied the most unstable mode of a BEC for different trapping geometries showing a crossover from a phonon-to a roton unstable system. This mode becomes increasingly local. This can be seen in the density profiles during the first stages of the post-destabilization dynamics.

Let us now outline some possible directions for future work. First our approach is based on the local density approximation and is therefore not able to describe systems with large trap aspect ratios which undergo rotonic collapses being hence difficult to assess the effects of quantum fluctuations in the phononto-roton crossover discussed in chapter 3. A more general way of calculating the effects of quantum fluctuations has to be found. In principal this can be achieved in a systematic way by expanding the Hamiltonian to higher orders in the non-condensed part of the field operator. By means of the Wick contraction and averaging over the Hamiltonian one can derive coupled equations for the correlation functions which take the effects of quantum fluctuations into account without the local density approximation. However, anomalous pair correlations stemming from the dipole-dipole interaction $\left\langle\psi^{\dagger}(\mathbf{r}) \psi\left(\mathbf{r}^{\prime}\right)\right\rangle$ can not be calculated efficiently with the current techniques. An efficient way to solve these equations is required to study the effects of quantum fluctuations for arbitrary parameter regimes.

A possible solution for this problem is to use a truncated Wigner approximation. This is a method in which the time evolution is governed by quasi-classical equations like the Gross-Pitaevskii equation. The effect of the quantum fluctuations is then taken into account by performing many calculations with different initial conditions which deviate by random fluctuations taken from a specific probability distribution. Statistical averaging over the different realizations gives the result incorporating quantum fluctuations. This technique is not based on the local density approximation and should be applicable for all parameter regimes. However it remains an open question if these systems close to instability can be correctly described by this method.

Moreover, the effects of quantum fluctuations can be studied in quasi-low dimensional systems. An initial investigation shows that the stable quantum droplets can be created in a 1D geometry albeit in a different manner as in 3D. Quantum fluctuations provide in 1D an effective attraction, in stark contrast to the 3D case. As a results the LHY correction may allow for the observation of self-bound droplets even when the mean-field term is repulsive. A detailed and refined study of $1 \mathrm{D}$ and $2 \mathrm{D}$ systems has to be done in order to understand the underlying physics and to show the qualitatively and quantitatively different behavior of these systems.

Quantum fluctuations play also an important role for the three-body losses as they influence the loss rate in a similar manner as the interactions. Especially in 
systems with strong losses as in recent erbium experiments quantum fluctuations can significantly change the losses and therefore alter the dynamics substantially. This study can be expanded to quasi low-dimensional systems. Especially since the LHY term is known to be negative in a 1D system. A systematic study on how quantum fluctuations affect three-body losses in lower dimensional dipolar gases is still to be performed. 


\section{A. Validity of the local density approximation}

As explained in section 4.4 the effect of quantum fluctuations in inhomogeneous systems can be described within the local density approximation when the main contribution to the LHY term stems from modes whose wavelength is much smaller than the condensate size $R$. This condition is automatically fulfilled in the Thomas-Fermi regime $[144,145]$. In this chapter we adress the validity of the LDA for the small droplets that are created in dysprosium experiments forming the crystal-like structures as well as the droplets formed in erbium condensates. We start with the discussion of the dysprosium droplets.

The energy shift due to quantum fluctuations was derived in section 4.1 and yields

$$
\Delta E_{0}=\frac{1}{2} \sum_{\mathbf{p} \neq 0}\left(\epsilon(\mathbf{p})-\frac{\mathbf{p}^{2}}{2 M}-n \tilde{V}_{\mathrm{cd}}(\mathbf{p})+\frac{M \tilde{V}_{\mathrm{cd}}(-\mathbf{p}) \tilde{V}_{\mathrm{cd}}(\mathbf{p}) n^{2}}{\mathbf{p}^{2}}\right)
$$

Here $\tilde{V}_{\text {cd }}(\mathbf{p})$ includes both the contact and the dipolar potential. Substituting the sum by an integral via $\sum_{\mathbf{p}} \rightarrow \frac{V}{(2 \pi \hbar)^{3}} \int d^{3} p$ and switching to spherical coordinates in the integrand leads to

$$
\begin{aligned}
\Delta E_{0}= & \frac{1}{2} \frac{V}{(2 \pi)^{3}} \int_{0}^{\infty} d k k^{2} \int_{0}^{\pi} d \theta \sin \theta \int_{0}^{2 \pi} d \varphi \times \\
& \times\left(\epsilon(k)-\frac{\hbar^{2} k^{2}}{2 M}-g n f\left(\epsilon_{\mathrm{dd}}, \theta\right)+\frac{M g^{2} n^{2} f\left(\epsilon_{\mathrm{dd}}, \theta\right)^{2}}{\hbar^{2} k^{2}}\right),
\end{aligned}
$$

with $\epsilon(\mathbf{k})=\sqrt{\frac{\hbar^{2} k^{2}}{2 M}\left(\frac{\hbar^{2} k^{2}}{2 M}+2 g n f\left(\epsilon_{\mathrm{dd}}, \theta\right)\right)}$ being the Bogoliubov spectrum of a dipolar BEC, $f\left(\epsilon_{\mathrm{dd}}, \theta\right)=1+\epsilon_{\mathrm{dd}}\left(3 \cos ^{2} \theta-1\right)$ and we also introduced $k=p / \hbar$. We rescale the integrand in units of the healing length $\xi=\hbar / \sqrt{2 M g n}$ and integrate out the $\varphi$-direction which leads to

$\Delta E_{0}=\frac{V(2 M g n)^{\frac{3}{2}} g n}{2(2 \pi)^{2} \hbar^{3}} \int_{0}^{\pi} d \theta \sin \theta \int_{0}^{\infty} d k k^{2}\left(\tilde{\epsilon}(k)-k^{2}-f\left(\epsilon_{\mathrm{dd}}, \theta\right)+\frac{f\left(\epsilon_{\mathrm{dd}}, \theta\right)^{2}}{2 k^{2}}\right)$,

where $\tilde{\epsilon}(k)=\sqrt{k^{2}\left(k^{2}+2 f\left(\epsilon_{\mathrm{dd}}, \theta\right)\right)}$. The integration in momentum space ranges from 0 to $\infty$ which incorporates also modes with small momenta whose wavelengths are comparable or even larger than the condensate size. These modes are not described well within LDA. In order to investigate the relevance of these modes we consider a low-momentum cutoff of the form $q_{c}(\theta)=q_{z}\left(\cos ^{2} \theta\right.$ $\left.+\kappa^{2} \sin ^{2} \theta\right)^{1 / 2}$, which incorporates the cylindrical symmetry of the droplet with $\kappa$ being the aspect ratio of the droplet and $q_{z}$ the cutoff along the $z$-direction. 
Introducing this cutoff in the integral of Eq. (A.3) results in a modified LHY correction. The corrected chemical potential then reads

$$
\Delta \mu_{c}=\frac{5(2 M g n)^{3 / 2} g}{4(2 \pi)^{2} \hbar^{3}} \int_{0}^{\pi} d \theta \sin \theta \int_{q_{c}(\theta)}^{\infty} d k k^{2}\left(\epsilon(k)-k^{2}-f\left(\epsilon_{\mathrm{dd}}, \theta\right)+\frac{f\left(\epsilon_{\mathrm{dd}}, \theta\right)^{2}}{2 k^{2}}\right) .
$$

We compare the result of Eq. (A.4) with the LHY correction calculated without the cut-off which reads

$$
\frac{\Delta \mu_{c}}{\Delta \mu}=\frac{15 \sqrt{2}}{16} \frac{\int_{0}^{\pi} d \theta \sin \theta \chi\left(\epsilon_{\mathrm{dd}}, \theta\right)}{\int_{0}^{\pi} d \theta \sin \theta f\left(\epsilon_{\mathrm{dd}}, \theta\right)^{5 / 2}},
$$

where we integrated out the $k$-direction and

$$
\begin{aligned}
\chi\left(\epsilon_{\mathrm{dd}}\right)= & 2^{5 / 2}\left(\frac{2 f\left(\epsilon_{\mathrm{dd}}, \theta\right)}{15}-\frac{q_{c}(\theta)^{2}}{10}\right)\left(\frac{q_{c}(\theta)^{2}}{2}+f\left(\epsilon_{\mathrm{dd}}, \theta\right)\right)^{3 / 2} \\
& +\frac{q_{c}(\theta)^{5}}{5}+\frac{q_{c}(\theta)^{3}}{3} f\left(\epsilon_{\mathrm{dd}}, \theta\right)-\frac{q_{c}(\theta)}{2} f\left(\epsilon_{\mathrm{dd}}, \theta\right)^{2} .
\end{aligned}
$$

Let us consider a typical droplet created in the crystal-like structures in dysprosium with $N=1000$ at a scattering length of $a_{s}=70 a_{B}$. The size of the condensate in $z$-direction is $\simeq 2 \mu \mathrm{m}$ which corresponds to $\simeq 25 \xi$ with $\xi$ calculated for an averaged central density of $1.5 \times 10^{21} \mathrm{~m}^{-3}$ and the droplet aspect ratio is given by $\kappa \simeq 6$. For a $z$-cutoff $q_{z} \xi \simeq 0.25$, excitations with $|\mathbf{q}(\theta)|>q_{c}(\theta)$ may be considered as having a wavelength much smaller than the droplet size. For this cutoff, we obtain $\frac{\Delta \mu_{c}}{\Delta \mu} \simeq 0.8$, showing a large contribution of short-wavelength excitations. The corrections due to long-wavelength modes may modify the prefactor of the LHY correction, but the bulk of the effect is well recovered in the dysprosium experiments validating Eq. (5.2).

The situation is even better for the droplets discussed in erbium. Here for typical experimental parameters, e.g. $a_{s}=58 a_{B}, N=5 \times 10^{4}$, we find an axial radius $R_{z}=13 \mu \mathrm{m}$ and $\kappa=7.5$. Then we can introduce even a 4 times larger cutoff radius $q_{z} \xi \simeq 1$ leading to the same factor $\frac{\Delta \mu_{c}}{\Delta \mu} \simeq 0.8$, demonstrating that short-wavelength excitations indeed are the dominant contribution in the erbium experiments and that, hence the numerical methods should match even better to the erbium experiments as shown in chapter 7 . 


\section{B. Numerical procedure to calculate the Bogoliubov spectrum}

In this chapter we present in detail the numerical procedure of the calculation of the Bogoliubov modes for chapter 3 and the derivation of the effective momentum. As discussed in section 3.2 we introduced the new functions $f_{+}$and $f_{-}$as linear combinations of the Bogoliubov amplitudes $u$ and $v$. The resulting equations for $f_{+}$and $f_{-}$(Eqs. (3.5) and (3.7)) are then projected into a harmonic oscillator basis leading to linear equations for the expansion coefficients (see section 3.2). The resulting linear equations for the expansion coefficients $c^{+}$and $c^{-}$are then solved by LAPACK routines. The remaining task is to calculate the matrix elements of the linear equations.

\section{B.1. Matrix Elements for $f_{+}$}

After projecting Eq. (3.7) into the harmonic oscillator basis we obtain $E^{2} v=\hat{M}^{+} v$ which is an eigenvalue equation for the matrix $\hat{M}^{+}$with $E^{2}$ being the eigenvalue to the eigenvector $v$. The matrix elements of $\hat{M}^{+}$read

$$
\begin{aligned}
M_{k m i l n j}^{+} & =\int d^{3} r \psi_{l n j}^{*}(\mathbf{r}) \hat{M}^{+} \psi_{k m i}(\mathbf{r})=\int d^{3} r \psi_{l n j}^{*}(\mathbf{r})\left(\hat{H}_{0}-\mu\right)^{2} \psi_{k m i}(\mathbf{r}) \\
& +\int d^{3} r \psi_{l n j}^{*}(\mathbf{r})\left(\hat{H}_{0}-\mu\right)[3 g n(\mathbf{r})+I(\mathbf{r})] \psi_{k m i}(\mathbf{r}) \\
& +\int d^{3} r \psi_{l n j}^{*}(\mathbf{r})[g n(\mathbf{r})+I(\mathbf{r})]\left[\hat{H}_{0}-\mu+3 g n(\mathbf{r})+I(\mathbf{r})\right] \psi_{k m i}(\mathbf{r}) \\
& +\int d^{3} r \psi_{l n j}^{*}(\mathbf{r})\left[\hat{H}_{0}-\mu+g n(\mathbf{r})+I(\mathbf{r})\right] \times \\
& \times 2 \psi(\mathbf{r}) \int d^{3} r^{\prime} V_{\mathrm{dd}}\left(\mathbf{r}-\mathbf{r}^{\prime}\right) \psi\left(\mathbf{r}^{\prime}\right) \psi_{k m i}\left(\mathbf{r}^{\prime}\right)
\end{aligned}
$$

where $I(\mathbf{r})=\int d^{3} r^{\prime} V_{\mathrm{dd}}\left(\mathbf{r}-\mathbf{r}^{\prime}\right) n\left(\mathbf{r}^{\prime}\right)$ and $\hat{H}_{0}=-\frac{\hbar^{2} \nabla^{2}}{2 M}+\frac{M}{2}\left(\omega_{\perp}^{2} \rho^{2}+\omega_{z}^{2} z^{2}\right)$ is the Hamiltonian of the non-interacting system and the $\psi_{k m i}$ are the harmonic oscillator basis functions. We will adress each term individually starting with the first line. The action of $\hat{H}_{0}$ onto $\psi_{k m i}(\mathbf{r})$ is known $\hat{H}_{0} \psi_{k m i}=E_{k m i} \psi_{k m i}$, so that the first term yields

$$
\int d^{3} r \psi_{l n j}^{*}(\mathbf{r})\left(\hat{H}_{0}-\mu\right)^{2} \psi_{k m i}(\mathbf{r})=\left(E_{k m i}-\mu\right)^{2} \delta_{k l} \delta_{m n} \delta_{i j} .
$$


The second line can be simplified by noting that $\hat{H}_{0}$ is Hermitean and can therefore also act onto the left side, which yields

$$
\begin{aligned}
& \int d^{3} r \psi_{l n j}^{*}(\mathbf{r})\left(\hat{H}_{0}-\mu\right)[3 g n(\mathbf{r})+I(\mathbf{r})] \psi_{k m i}(\mathbf{r}) \\
= & \left(E_{l n j}-\mu\right) \int d^{3} r \psi_{l n j}^{*}(\mathbf{r})[3 g n(\mathbf{r})+I(\mathbf{r})] \psi_{k m i}(\mathbf{r}) .
\end{aligned}
$$

The term $3 g n(\mathbf{r})+I(\mathbf{r})$ is cylindrically symmetric so that we can integrate out the angle in the xy plane which leads to

$$
\begin{aligned}
& \int_{0}^{\infty} d \rho \rho \int_{-\infty}^{\infty} d z \psi_{l n}(\rho) \psi_{k m}(\rho) \psi_{i}(z) \psi_{j}(z)[3 g n(\rho, z)+I(\rho, z)] \underbrace{\int_{0}^{2 \pi} d \phi \mathrm{e}^{-\mathrm{i}(n-m) \phi}}_{=2 \pi \delta_{n m}} \\
& =2 \pi \delta_{n m} \int_{0}^{\infty} d \rho \rho \psi_{l n}(\rho) \psi_{k m}(\rho) \int_{-\infty}^{\infty} d z \psi_{i}(z) \psi_{j}(z)[3 g n(\rho, z)+I(\rho, z)] .
\end{aligned}
$$

The remaining 2D integral can be calculated efficiently using a third order method. The dipole integral $I(\mathbf{r})$ has to be calculated once and this is done via Fourier transformation as explained in section 3.1. The third term can be calculated analogously and yields

$$
\begin{aligned}
\int d^{3} r \psi_{l n j}^{*}(\mathbf{r})[ & g n(\mathbf{r})+I(\mathbf{r})]\left[\hat{H}_{0}-\mu+3 g n(\mathbf{r})+I(\mathbf{r})\right] \psi_{k m i}(\mathbf{r}) \\
= & 2 \pi \delta_{m n} \int d \rho d z \rho \psi_{k m}(\rho) \psi_{l n}(\rho) \psi_{i}(z) \psi_{j}(z)[g n(\rho, z)+I(\rho, z)] \times \\
& \times\left[E_{k m i}-\mu+3 g n(\rho, z)+I(\rho, z)\right] .
\end{aligned}
$$

The calculation of the fourth term is more elaborate. We perform the integration in momentum space in order to avoid problems with the dipole-dipole potential. Let us first calculate the Fourier transform of a function which is cylindrically symmetric except for a factor $\mathrm{e}^{\mathrm{i} m \phi}$ and, for definiteness, can be written as the product $\psi_{k m}(\rho) e^{\mathrm{i} m \phi} \psi_{i}(z)$ which leads to

$$
\begin{aligned}
\hat{\psi}_{k m i}(\mathbf{k}) & =\int d^{3} r \psi_{k m i}(\mathbf{r}) \mathrm{e}^{-\mathrm{i} \mathbf{k} \cdot \mathbf{r}} \\
& =\int_{0}^{\infty} d \rho \rho \int_{0}^{2 \pi} d \phi \int_{-\infty}^{\infty} d z \psi_{k m}(\rho) \mathrm{e}^{\mathrm{i} m \phi} \mathrm{e}^{-\mathrm{i} k \rho \cos \left(\phi-\vartheta_{k}\right)} \mathrm{e}^{-\mathrm{i} k_{z} z} \psi_{i}(z),
\end{aligned}
$$

where we transformed both the spatial and the Fourier coordinates into cylinder coordinates with $\vartheta_{k}$ being the angular component of $\mathbf{k}$. After Fourier transforming the $z$-component and substituting $\phi-\vartheta_{k}=\phi^{\prime}$ we obtain

$$
\hat{\psi}_{k m i}(\mathbf{k})=\hat{\psi}_{i}\left(k_{z}\right) \int_{0}^{\infty} d \rho \rho \psi_{k m}(\rho) \int_{0}^{2 \pi} d \phi^{\prime} \mathrm{e}^{\mathrm{i} m \phi^{\prime}} \mathrm{e}^{\mathrm{i} m \vartheta_{k}} \mathrm{e}^{-\mathrm{i} k \rho \cos \phi^{\prime}}
$$

The $\phi^{\prime}$-integral can be solved analytically which yields

$$
\int_{0}^{2 \pi} d \phi^{\prime} \mathrm{e}^{\mathrm{i} m \phi^{\prime}} \mathrm{e}^{\mathrm{i} a \cos \phi^{\prime}}=2 \pi \mathrm{i}^{m} J_{m}(a),
$$


leading to

$$
\hat{\psi}_{k m i}(\mathbf{k})=2 \pi \mathrm{i}^{m} \hat{\psi}_{i}\left(k_{z}\right) \mathrm{e}^{\mathrm{i} m \vartheta_{k}} \int_{0}^{\infty} d \rho \rho \psi_{k m}(\rho) J_{m}(-k \rho) .
$$

Using that $J_{m}(-x)=J_{-m}(x)=(-1)^{m} J_{m}(x)$ yields the final result

$$
\hat{\psi}_{k m i}(\mathbf{k})=2 \pi \mathrm{i}^{3 m} \hat{\psi}_{i}\left(k_{z}\right) \mathrm{e}^{\mathrm{i} m \vartheta_{k}} \hat{\psi}_{k m}(k),
$$

where $\hat{\psi}_{k m}(k)=\int_{0}^{\infty} d \rho \rho \psi_{k m}(\rho) J_{m}(k \rho)$. The result for the complex conjugate reads

$$
\hat{\psi}_{k m i}(\mathbf{k})^{*}=2 \pi \mathrm{i}^{m} \hat{\psi}_{i}\left(k_{z}\right) \mathrm{e}^{-\mathrm{i} m \vartheta_{k}} \hat{\psi}_{k m}(k) .
$$

Now we come back to the fourth term of the calculation of the matrix elements by noting that its form is given by

$$
I_{4}=2 \int d^{3} r f_{l n j}^{*}(\mathbf{r}) \int d^{3} r^{\prime} V_{\mathrm{dd}}\left(\mathbf{r}-\mathbf{r}^{\prime}\right) g_{k m i}\left(\mathbf{r}^{\prime}\right),
$$

where $f_{l n j}^{*}(\mathbf{r})=\psi_{l n j}^{*}(\mathbf{r})\left[E_{l n j}-\mu+g n(\mathbf{r})+I(\mathbf{r})\right] \psi(\mathbf{r})$ and $g_{k m i}(\mathbf{r})=\psi(\mathbf{r}) \psi_{k m i}(\mathbf{r})$ are abbreviations and cylindrically symmetric except for a factor $e^{ \pm i m \phi}$. Therefore the Fourier transform of the integrand can be calculated leading to a cylindersymmetric function and the $\vartheta_{k}$-direction can be integrated out which leads to

$$
I_{4}=2 \int d k d k_{z} k \tilde{f}_{k m i}\left(k, k_{z}\right) \tilde{V}_{\mathrm{dd}}\left(k, k_{z}\right) \tilde{g}_{l n j}\left(k, k_{z}\right) \text {. }
$$

The 2D integration in momentum space is again implemented by a third order method. The Fourier transforms are calculated using the fast Fourier transform routine FFTW3 [157]. The integration over the Bessel function is done via an adaptive step size routine including a linear interpolation routine.

\section{B.2. Calculating the Matrix Elements for $f_{-}$}

The techniques to calculate the matrix elements for the projected Eq. (3.5) for the function $f_{-}$are the same as in the case for $f_{+}$. We expand the function $f_{-}$ in terms of harmonic oscillator basis states thereby introducing new expansion coefficients $c^{-}$

$$
f_{-}=\sum_{k m i} c_{k m i}^{-} \psi_{k m i}
$$

Projecting Eq. (3.5) leads to

$$
E_{l n j} c_{l n j}^{+}=\underbrace{\int d^{3} r \psi_{l n j}^{*}(\mathbf{r}) \hat{M}^{-} \sum_{k m i} c_{k m i}^{-} \psi_{k m i}(\mathbf{r})}_{=\sum_{k m i} M_{k m i l n j}^{-} c_{k m i}^{-}},
$$

where

$$
M_{k m i l n j}^{-}=-\int d^{3} r \psi_{l n j}^{*}(\mathbf{r})\left[-\frac{\hbar^{2} \nabla^{2}}{2 M}+V_{\mathrm{tr}}(\mathbf{r})-\mu+g n(\mathbf{r})+I(\mathbf{r})\right] \psi_{k m i}(\mathbf{r}),
$$


which can be simplified to

$$
\begin{aligned}
M_{k m i l n j}^{-}= & -\left(E_{k m i}-\mu\right) \delta_{k l} \delta_{m n} \delta_{i j} \\
& -2 \pi \delta_{m n} \int d \rho \rho \int d z \psi_{k m}(\rho) \psi_{i}(z) \psi_{l n}(\rho) \psi_{j}(z)[g n(\mathbf{r})+I(\mathbf{r})]
\end{aligned}
$$

With these results the Bogoliubov modes can be calculated by first performing an imaginary time evolution of Eq. (3.1). Then we calculate the matrix elements of the matrix $\hat{M}^{+}$, where we parallelize the calculation of the individual elements. Afterwards we solve the eigenvalue equation for $f_{+}$and obtain the squared of the energy of the modes as well as $f_{+}$described by the expansion coefficients $c^{+}$. We calculate then the matrix elements $M^{-}$and solve the corresponding set of linear equations to obtain the coefficients $c^{-}$. The calculations are done for a specific quantum number $m$ enabling an additional parallelization. The effective momentum is then calculated directly from the expansion coefficients as shown in the next section.

\section{B.3. Calculating the effective momentum}

Let us start with the definition of the effective momentum which is given in Eq. (3.14). We rewrite the integrand in position space which reads

$$
k_{\mathrm{eff}}^{2}=\int d^{3} r\left[u^{*}(\mathbf{r}) \nabla_{\perp}^{2} u(\mathbf{r})+v(\mathbf{r}) \nabla_{\perp}^{2} v^{*}(\mathbf{r})\right] .
$$

Inserting the definitions of $u$ and $v$ in terms of $f^{+}$and $f^{-}$leads to

$$
\begin{aligned}
k_{\mathrm{eff}}^{2}= & \frac{1}{4} \int d^{3} r\left[f_{+}^{*} \nabla_{\perp}^{2} f_{+}-f_{-}^{*} \nabla_{\perp}^{2} f_{+}-f_{+}^{*} \nabla_{\perp}^{2} f_{-}+f_{-}^{*} \nabla_{\perp}^{2} f_{-}\right. \\
& \left.f_{+} \nabla_{\perp}^{2} f_{+}^{*}+f_{+} \nabla_{\perp}^{2} f_{-}^{*}+f_{-} \nabla_{\perp}^{2} f_{+}^{*}+f_{-} \nabla_{\perp}^{2} f_{-}^{*}\right] .
\end{aligned}
$$

This can be simplified as the trivial $\phi$-dependence of these terms can be integrated out leading to

$$
\begin{aligned}
\int d^{3} r\left[f_{ \pm}^{*} \nabla_{\perp}^{2} f_{ \pm}+f_{ \pm} \nabla_{\perp}^{2} f_{ \pm}^{*}\right]= & 4 \pi \int d \rho \rho \int d z \sum_{k m i} \sum_{l n j} \delta_{m, n} c_{k m i}^{ \pm} c_{l n j}^{ \pm} \\
& \times \psi_{k m} \psi_{i}\left(\frac{\partial^{2}}{\partial \rho^{2}}+\frac{1}{\rho} \frac{\partial}{\partial \rho}-\frac{n^{2}}{\rho^{2}}\right) \psi_{l n} \psi_{j}
\end{aligned}
$$

and

$$
\begin{aligned}
& \int d^{3} r\left[f_{-} \nabla_{\perp}^{2} f_{+}^{*}-f_{-}^{*} \nabla_{\perp}^{2} f_{+}\right]=0, \\
& \int d^{3} r\left[f_{+} \nabla_{\perp}^{2} f_{-}^{*}-f_{+}^{*} \nabla_{\perp}^{2} f_{-}\right]=0 .
\end{aligned}
$$

Putting this together yields

$$
\begin{aligned}
k_{\text {eff }}^{2}= & -\sum_{k m i} \sum_{l n j} \pi \delta_{m n} \int d \rho \rho \int d z\left(c_{k m i}^{+} c_{l n j}^{+}+c_{k m i}^{-} c_{l n j}^{-}\right) \\
& \times \psi_{k m}(\rho) \psi_{i}(z)\left(\frac{\partial^{2}}{\partial \rho^{2}}+\frac{1}{\rho} \frac{\partial}{\partial \rho}-\frac{n^{2}}{\rho^{2}}\right) \psi_{l n}(\rho) \psi_{j}(z) .
\end{aligned}
$$


The derivatives can be rewritten as simple factors of $\rho^{2}$ and $z^{2}$ by using the differential equation for the harmonic basis functions

$$
\left(\frac{\partial^{2}}{\partial \rho^{2}}+\frac{1}{\rho} \frac{\partial}{\partial \rho}-\frac{n^{2}}{\rho^{2}}\right) \psi_{l n j}=\left(\rho^{2}-2 E_{l n}\right) \psi_{l n j} .
$$

Eq. (B.24) can be solved analytically. After the substitution $\rho^{2}=x$ we obtain

$$
\int_{0}^{\infty} d \rho \rho \psi_{k m}(\rho) \rho^{2} \psi_{l m}(\rho)=A_{k m} A_{l m} \int_{0}^{\infty} d x x^{|m|+1} \mathrm{e}^{-x} L_{k}^{|m|}(x) L_{l}^{|m|}(x)
$$

which can be solved by rewriting the Laguerre polynomials $L_{k}^{|m|}=L_{k}^{|m|+1}-L_{k-1}^{|m|+1}$ and using the integral $\int_{0}^{\infty} d x x^{\alpha} \mathrm{e}^{-x} L_{n}^{\alpha} L_{m}^{\alpha}=\frac{\Gamma(n+\alpha+1)}{n !} \delta_{n m}$ which yields

$$
\begin{aligned}
& \int_{0}^{\infty} d \rho \rho \psi_{k m}(\rho) \rho^{2} \psi_{l m}(\rho)=\frac{1}{2 \pi}\left[(2 k+|m|+1) \delta_{k, l}\right. \\
& \left.\quad-\sqrt{k+1} \sqrt{k+|m|+1} \delta_{k, l-1}-\sqrt{l+1} \sqrt{l+|m|+1} \delta_{l, k-1}\right] .
\end{aligned}
$$

Then, the effective momentum can be calculated by

$$
\begin{aligned}
k_{\mathrm{eff}}^{2}= & \sum_{k=0}^{N_{k}-1} \sum_{m i}\left(c_{k m i}^{+} c_{(k+1) m i}^{+}+c_{k m i}^{-} c_{(k+1) m i}^{-}\right) \sqrt{k+1} \sqrt{k+|m|+1} \\
& +\frac{1}{2} \sum_{k m i}\left[\left(c_{k m i}^{+}\right)^{2}+\left(c_{k m i}^{-}\right)^{2}\right] E_{k m i},
\end{aligned}
$$

where $N_{k}$ is the number of basis states $\psi_{k m}(\rho)$. 


\section{Bibliography}

[1] S. N. Bose, Plancks Gesetz und Lichtquantenhypothese, Z. Physik 26, 178 (1924).

[2] A. Einstein, Quantentheorie des einatomigen idealen Gases, Sitzber. Kgl. Preuss. Akad. Wiss. , 3 (1925).

[3] E. C. Svensson and V. F. Sears, Neutron Scattering by ${ }^{4} \mathrm{He}$ and ${ }^{3} \mathrm{He}$, Progress in Low Temperature Physics XI, 189 (1987).

[4] T. W. Hänsch and A. L. Schawlow, Cooling of gases by laser radiation, Opt. Commun. 13, 68 (1975).

[5] D. J. Wineland and W. M. Itano, Laser cooling of atoms, Phys. Rev. A 20, 1521 (1979).

[6] W. Ketterle and N. J. van Druten, Evaporative cooling of trapped atoms, Adv. At. Mol. Opt. Phys. 37, 181 (1996).

[7] M. H. Anderson, J. R. Ensher, M. R. Matthews, C. E. Wieman, and E. A. Cornell, Observation of Bose-Einstein Condensation in a Dilute Atomic Vapor, Science 269, 198 (1995).

[8] K. B. Davis, M.-O. Mewes, M. R. Andrews, N. J. van Druten, D. S. Durfee, D. M. Kurn, and W. Ketterle, Bose-Einstein Condensation in a Gas of Sodium Atoms, Phys. Rev. Lett. 75, 3969 (1995).

[9] E. Cornell, Very Cold Indeed: The Nanokelvin Physics of Bose-Einstein Condensation, J. Res. Natl. Inst. Stand. Technol. 101, 419 (1996).

[10] H. S. Walter Greiner, Ludwig Neise, Thermodynamik und Statistische Mechanik (Verlag Harri Deutsch, 1993).

[11] A. Griffin, D. W. Snoke, and S. Stringari, Bose-Einstein Condensation (Cambridge University Press, 1995).

[12] O. Penrose and L. Onsager, Bose-Einstein Condensation and Liquid Helium, Phys. Rev. 104, 576 (1956).

[13] J. Goldstone, Field theories with «Superconductor» solutions, N. Cim. 19, 154 (1961).

[14] C. N. Yang, Concept of Off-Diagonal Long-Range Order and the Quantum Phases of Liquid He and of Superconductors, Rev. Mod. Phys. 34, 694 (1962). 
[15] P. W. Anderson, Considerations on the Flow of Superfluid Helium, Rev. Mod. Phys. 38, 298 (1966).

[16] F. Schwabl, Quantenmechanik (Springer, 2007).

[17] L. Pitaevskii and S. Stringari, Bose-Einstein Condensation (Clarendon Press, 2003).

[18] C. J. Pethick and H. Smith, Bose-Einstein Condensation in Dilute Gases (Cambridge University Press, 2002).

[19] E. Fermi, Motion of neutrons in hydrogenous substances, Ric. Sci. 7, 13 (1936).

[20] C. Chin, R. Grimm, P. Julienne, and E. Tiesinga, Feshbach resonances in ultracold gases, Rev. Mod. Phys. 82, 1225 (2010).

[21] H. Feshbach, A unified theory of nuclear reactions. II, Annalen der Physik 19, 287 (1962).

[22] S. Inouye, M. R. Andrews, J. Stenger, H. J. Miesner, D. M. Stamper-Kurn, and W. Ketterle, Observation of Feshbach resonances in a Bose-Einstein condensate, Nature 392, 151 (1998).

[23] D. M. Bauer, M. Lettner, C. Vo, G. Rempe, and S. Dürr, Control of a magnetic Feshbach resonance with laser light, Nat. Phys. 5, 339 (2009).

[24] M. Theis, G. Thalhammer, K. Winkler, M. Hellwig, G. Ruff, R. Grimm, and J. H. Denschlag, Tuning the Scattering Length with an Optically Induced Feshbach Resonance, Phys. Rev. Lett. 93, 123001 (2004).

[25] http://cua.mit.edu/ketterle_group/experimental_setup/BEC_I/ Feshbach.jpg.

[26] F. Dalfovo, S. Giorgini, L. P. Pitaevskii, and S. Stringari, Theory of BoseEinstein condensation in trapped gases, Rev. Mod. Phys. 71, 463 (1999).

[27] N. N. Bogoliubov, On the theory of superfluidity, J. Phys. USSR 11, 23 (1947).

[28] E. P. Gross, Structure of a quantized vortex in boson systems, N. Cim. 20, 454 (1961).

[29] L. P. Pitaevskii, Vortex lines in an imperfect Bose gas, Sov. Phys. JETP 13, 451 (1961).

[30] http://www.aldebaran.cz/bulletin/2008_37/Boseno_sym.gif.

[31] E. A. Donley, N. R. Claussen, S. L. Cornish, J. L. Roberts, E. A. Cornell, and C. E. Wieman, Dynamics of collapsing and exploding Bose-Einstein condensates, Nature 412, 295 (2001).

[32] Y. Kagan, A. E. Muryshev, and G. V. Shlyapnikov, Collapse and BoseEinstein Condensation in a Trapped Bose Gas with Negative Scattering Length, Phys. Rev. Lett. 81, 933 (1998). 
[33] A. Griesmaier, J. Werner, S. Hensler, J. Stuhler, and T. Pfau, Bose-Einstein Condensation of Chromium, Phys. Rev. Lett. 94, 160401 (2005).

[34] M. Lu, N. Q. Burdick, S. H. Youn, and B. L. Lev, Strongly Dipolar BoseEinstein Condensate of Dysprosium, Phys. Rev. Lett. 107, 190401 (2011).

[35] K. Aikawa, A. Frisch, M. Mark, S. Baier, A. Rietzler, R. Grimm, and F. Ferlaino, Bose-Einstein Condensation of Erbium, Phys. Rev. Lett. 108, 210401 (2012).

[36] D. DeMille, Quantum Computation with Trapped Polar Molecules, Phys. Rev. Lett. 88, 067901 (2002).

[37] J. Deiglmayr, A. Grochola, M. Repp, K. Mörtlbauer, C. Glück, J. Lange, O. Dulieu, R. Wester, and M. Weidenmüller, Formation of Ultracold Polar Molecules in the Rovibrational Ground State, Phys. Rev. Lett. 101, 133004 (2008).

[38] K.-K. Ni, S. Ospelkaus, M. H. G. de Miranda, A. Pe'er, B. Neyenhuis, J. J. Zirbel, S. Kotochigova, P. S. Julienne, D. S. Jin, and J. Ye, A High Phase-Space-Density Gas of Polar Molecules, Science 322, 231 (2008).

[39] K.-K. Ni, S. Ospelkaus, D. Wang, G. Quéméner, B. Neyenhuis, M. H. G. de Miranda, J. L. Bohn, J. Ye, and D. S. Jin, Dipolar collisions of polar molecules in the quantum regime, Nature 464, 1324 (2010).

[40] L. D. Carr, D. DeMille, R. V. Krems, and J. Ye, Cold and ultracold molecules: science, technology and applications, New J. Phys. 11, 055049 (2009).

[41] S. Ospelkaus, K.-K. Ni, G. Quéméner, B. Neyenhuis, D. Wang, M. H. G. de Miranda, J. L. Bohn, J. Ye, and D. S. Jin, Controlling the Hyperfine State of Rovibronic Ground-State Polar Molecules, Phys. Rev. Lett. 104, 030402 (2010).

[42] S. Ospelkaus, K.-K. Ni, D. Wang, M. H. G. de Miranda, B. Neyenhuis, G. Quéméner, P. S. Julienne, J. L. Bohn, D. S. Jin, and J. Ye, QuantumState Controlled Chemical Reactions of Ultracold Potassium-Rubidium Molecules, Science 327, 853 (2010).

[43] W. Li, T. Pohl, J. M. Rost, S. T. Rittenhouse, H. R. Sadeghpour, J. Nipper, B. Butscher, J. B. Balewski, V. Bendkowsky, R. Löw, and T. Pfau, A Homonuclear Molecule with a Permanent Electric Dipole Moment, Science 334, 1110 (2011).

[44] M. H. G. de Miranda, A. Chotia, B. Neyenhuis, D. Wang, G. Quéméner, S. Ospelkaus, J. L. Bohn, J. Ye, and D. S. Jin, Controlling the quantum stereodynamics of ultracold bimolecular reactions, Nat. Phys. 7, 502 (2011).

[45] M. Zeppenfeld, B. G. U. Englert, R. Glöckner, A. Prehn, M. Mielenz, C. Sommer, L. D. van Buuren, M. Motsch, and G. Rempe, Sisyphus cooling of electrically trapped polyatomic molecules, Nature 491, 570 (2012). 
[46] A. Chotia, B. Neyenhuis, S. A. Moses, B. Yan, J. P. Covey, M. Foss-Feig, A. M. Rey, D. S. Jin, and J. Ye, Long-Lived Dipolar Molecules and Feshbach Molecules in a 3D Optical Lattice, Phys. Rev. Lett. 108, 080405 (2012).

[47] C.-H. Wu, J. W. Park, P. Ahmadi, S. Will, and M. W. Zwielein, Ultracold Fermionic Feshbach Molecules of ${ }^{23} \mathrm{Na}^{40} \mathrm{~K}$, Phys. Rev. Lett. 109, 085301 (2012).

[48] T. Takekoshi, M. Debatin, R. Rameshan, F. Ferlaino, R. Grimm, H.-C. Nägerl, C. R. Le Sueur, J. M. Hutson, P. S. Julienne, S. Kotochigova, and E. Tiemann, Towards the production of ultracold ground-state RbCs molecules: Feshbach resonances, weakly bound states, and the coupledchannel model, Phys. Rev. A 85, 032506 (2012).

[49] D. Tong, S. M. Farooqi, J. Stanojevic, S. Krishnan, Y. P. Zhang, R. Côté, E. E. Eyler, and P. L. Gould, Local Blockade of Rydberg Excitation in an Ultracold Gas, Phys. Rev. Lett. 93, 063001 (2004).

[50] R. Heidemann, U. Raitzsch, V. Bendkowsky, B. Butscher, R. Löw, and T. Pfau, Rydberg excitation of Bose-Einstein condensates, Phys. Rev. Lett. 100, 033601 (2008).

[51] T. F. Gallagher and P. Pillet, Dipole-Dipole interactions of Rydberg atoms, Adv. At. Mol. Opt. Phys. 56, 161 (2008).

[52] V. Bendkowsky, B. Butcher, J. Nipper, J. P. Shaffer, R. Löw, and T. Pfau, Observation of ultralong-range Rydberg molecules, Nature 458, 1005 (2009).

[53] G. Pupillo, A. Micheli, M. Boninsegni, I. Lesanovsky, and P. Zoller, Strongly Correlated Gases of Rydberg-Dressed Atoms: Quantum and Classical Dynamics, Phys. Rev. Lett. 104, 223002 (2010).

[54] H. Weimer, M. Müller, I. Lesanovsky, P. Zoller, and H. P. Büchler, A Rydberg quantum simulator, Nat. Phys. 6, 382 (2010).

[55] N. Henkel, R. Nath, and T. Pohl, Three-Dimensional Roton Excitations and Supersolid Formation in Rydberg-Excited Bose-Einstein Condensates, Phys. Rev. Lett. 104, 195302 (2010).

[56] P. Schauß, M. Cheneau, M. Endres, T. Fukuhara, S. Hild, A. Omran, T. Pohl, C. Gross, S. Kuhr, and I. Bloch, Observation of spatially ordered structures in a two-dimensional Rydberg gas, Nature 491, 87 (2012).

[57] T. Peyronel, O. Firstenberg, Q.-Y. Liang, S. Hofferberth, A. V. Gorshkov, T. Pohl, M. D. Lukin, and V. Vuletić, Quantum nonlinear optics with single photons enabled by strongly interacting atoms, Nature 488, 57 (2012).

[58] R. Löw, H. Weimer, J. Nipper, J. B. Balewski, B. Butscher, H. P. Büchler, and T. Pfau, An experimental and theoretical guide to strongly interacting Rydberg gases, J. Phys. B: At. Mol. Opt. Phys. 45, 113001 (2012). 
[59] K. Góral, K. Rzążewski, and T. Pfau, Bose-Einstein condensation with magnetic dipole-dipole forces, Phys. Rev. A 61, 051601 (2000).

[60] J. Kim, B. Friedrich, D. P. Katz, D. Patterson, J. D. Weinstein, R. DeCarvalho, and J. M. Doyle, Buffer-Gas Loading and Magnetic Trapping of Atomic Europium, Phys. Rev. Lett. 78, 3665 (1997).

[61] J. D. Weinstein, R. deCarvalho, J. Kim, D. Patterson, B. Friedrich, and J. M. Doyle, Magnetic trapping of atomic chromium, Phys. Rev. A 57, R3173(R) (1998).

[62] S. Yi and L. You, Trapped atomic condensates with anisotropic interactions, Phys. Rev. A 61, 041604(R) (2000).

[63] S. Yi and L. You, Trapped condensates of atoms with dipole interactions, Phys. Rev. A 63, 053607 (2001).

[64] L. Santos, G. V. Shlyapnikov, P. Zoller, and M. Lewenstein, Bose-Einstein Condensation in Trapped Dipolar Gases, Phys. Rev. Lett. 85, 1791 (2000).

[65] K. Góral and L. Santos, Ground state and elementary excitations of single and binary Bose-Einstein condensates of trapped dipolar gases, Phys. Rev. A 66, 023613 (2002).

[66] L. Santos, G. V. Shlyapnikov, and M. Lewenstein, Roton-Maxon Spectrum and Stability of Trapped Dipolar Bose-Einstein Condensates, Phys. Rev. Lett. 90, 250403 (2003).

[67] J. Stuhler, A. Griesmaier, T. Koch, M. Fattori, T. Pfau, S. Giovanazzi, P. Pedri, and L. Santos, Observation of a Dipole-Dipole Interaction in a Degenerate Quantum Gas, Phys. Rev. Lett. 95, 150406 (2005).

[68] T. Lahaye, T. Koch, B. Fröhlich, M. Fattori, J. Metz, A. Griesmaier, S. Giovanazzi, and T. Pfau, Strong dipolar effects in a quantum ferrofluid, Nature 448, 672 (2007).

[69] T. Koch, T. Lahaye, J. Metz, B. Fröhlich, A. Griesmaier, and T. Pfau, Stabilization of a purely dipolar quantum gas against collapse, Nat. Phys. 4, 218 (2008).

[70] T. Lahaye, J. Metz, B. Frölich, T. Koch, M. Meister, A. Griesmaier, T. Pfau, H. Saito, Y. Kawaguchi, and M. Ueda, d-Wave Collapse and Explosion of a Dipolar Bose-Einstein Condensate, Phys. Rev. Lett. 101, 080401 (2008).

[71] Q. Beaufils, R. Chicireanu, T. Zanon, B. Laburthe-Tolra, E. Maréchal, L. Vernac, J.-C. Keller, and O. Gorceix, All-optical production of chromium Bose-Einstein condensates, Phys. Rev. A 77, 061601(R) (2008).

[72] G. Bismut, B. Pasquiou, E. Maréchal, P. Pedri, L. Vernac, O. Gorceix, and B. Laburthe-Tolra, Collective Excitations of a Dipolar Bose-Einstein Condensate, Phys. Rev. Lett. 105, 040404 (2012). 
[73] B. Pasquiou, E. Maréchal, G. Bismut, P. Pedri, L. Vernac, O. Gorceix, and B. Laburthe-Tolra, Spontaneous Demagnetization of a Dipolar Spinor Bose Gas in an Ultralow Magnetic Field, Phys. Rev. Lett. 106, 255303 (2011).

[74] B. Pasquiou, E. Maréchal, L. Vernac, O. Gorceix, and B. Laburthe-Tolra, Thermodynamics of a Bose-Einstein Condensate with Free Magnetization, Phys. Rev. Lett. 108, 045307 (2012).

[75] G. Bismut, B. Laburthe-Tolra, E. Maréchal, P. Pedri, O. Gorceix, and L. Vernac, Anisotropic Excitation Spectrum of a Dipolar Quantum Bose Gas, Phys. Rev. Lett. 109, 155302 (2012).

[76] H. Kadau, M. Schmitt, M. Wenzel, C. Wink, T. Maier, I. Ferrier-Barbut, and T. Pfau, Observing the Rosensweig instability of a quantum ferrofluid, Nature(London) 530, 194 (2016).

[77] I. Ferrier-Barbut, H. Kadau, M. Schmitt, M. Wenzel, and T. Pfau, Observation of Quantum Droplets in a Strongly Dipolar Bose Gas, Phys. Rev. Lett. 116, 215301 (2016).

[78] M. Schmitt, M. Wenzel, F. Böttcher, I. Ferrier-Barbut, and T. Pfau, Selfbound droplets of a dilute magnetic quantum liquid, arXiv:1607.07355v1 (2016).

[79] O. Dutta and P. Meystre, Ground-state structure and stability of dipolar condensates in anisotropic traps, Phys. Rev. 75, 053604 (2007).

[80] T. Lahaye, T. Pfau, and L. Santos, Mesoscopic Ensembles of Polar Bosons in Triple-Well Potentials, Phys. Rev. Lett. 104, 170404 (2010).

[81] A. Bühler and H. P. Büchler, Supersolid phase in atomic gases with magnetic dipole interaction, Phys. Rev. A 84, 023607 (2011).

[82] R. M. Wilson and J. L. Bohn, Emergent structure in a dipolar Bose gas in a one-dimensional lattice, Phys. Rev. A 83, 023623 (2011).

[83] A. Maluckov, G. Gligorić, L. Hadžievski, B. A. Malomed, and T. Pfau, Stable Periodic Density Waves in Dipolar Bose-Einstein Condensates Trapped in Optical Lattices, Phys. Rev. Lett. 108, 140402 (2012).

[84] S. Ronen, D. C. E. Bortolotti, and J. L. Bohn, Bogoliubov modes of a dipolar condensate in a cylindrical trap, Phys. Rev. A 74, 013623 (2006).

[85] S. Ronen, D. C. E. Bortolotti, and J. L. Bohn, Radial and Angular Rotons in Trapped Dipolar Gases, Phys. Rev. Lett. 98, 030406 (2007).

[86] R. M. Wilson, S. Ronen, J. L. Bohn, and H. Pu, Manifestations of the Roton Mode in Dipolar Bose-Einstein Condensates, Phys. Rev. Lett. 100, 245302 (2008).

[87] P. B. Blakie, D. Baillie, and R. N. Bisset, Roton spectroscopy in a harmonically trapped dipolar Bose-Einstein condensate, Phys. Rev. A 86, 021604(R) (2012). 
[88] R. N. Bisset, D. Baillie, and P. B. Blakie, Roton excitations in a trapped dipolar Bose-Einstein condensate, Phys. Rev. A 88, 043606 (2013).

[89] M. Jona-Lasinio, K. Łakomy, and L. Santos, Roton confinement in trapped dipolar Bose-Einstein condensates, Phys. Rev. A 88, 013619 (2013).

[90] R. M. Wilson, S. Ronen, and J. L. Bohn, Critical Superfluid Velocity in a Trapped Dipolar Gas, Phys. Rev. Lett. 104, 094501 (2010).

[91] C. Ticknor, R. M. Wilson, and J. L. Bohn, Anisotropic Superfluidity in a Dipolar Bose Gas, Phys. Rev. Lett. 106, 065301 (2011).

[92] D. C. E. Bortolotti, S. Ronen, J. L. Bohn, and D. Blume, Scattering Length Instability in Dipolar Bose-Einstein Condensates, Phys. Rev. Lett. 97, 160402 (2006).

[93] R. Nath, P. Pedri, and L. Santos, Phonon Instability with Respect to Soliton Formation in Two-Dimensional Dipolar Bose-Einstein Condensates, Phys. Rev. Lett. 102, 050401 (2009).

[94] R. M. Wilson, S. Ronen, and J. L. Bohn, Angular collapse of dipolar BoseEinstein condensates, Phys. Rev. A 80, 023614 (2009).

[95] S. Müller, J. Billy, E. A. L. Henn, H. Kadau, A. Griesmaier, M. JonaLasinio, L. Santos, and T. Pfau, Stability of a dipolar Bose-Einstein condensate in a one-dimensional lattice, Phys. Rev. A 84, 053601 (2011).

[96] J. Billy, E. A. L. Henn, S. Müller, T. Maier, H. Kadau, A. Griesmaier, M. Jona-Lasinio, and L. Santos, Deconfinement-induced collapse of a coherent array of dipolar Bose-Einstein condensates, Phys. Rev. A 86, 051603 (2012).

[97] N. R. Cooper, E. H. Rezayi, and S. H. Simon, Vortex Lattices in Rotating Atomic Bose Gases with Dipolar Interactions, Phys. Rev. Lett. 95, 200402 (2005).

[98] J. Zhang and H. Zhai, Vortex lattices in Planar Bose-Einstein Condensates with Dipolar Interactions, Phys. Rev. Lett. 95, 200403 (2005).

[99] S. Yi and H. Pu, Vortex structures in dipolar condensates, Phys. Rev. A 73, 061602(R) (2006).

[100] S. Komineas and N. R. Cooper, Vortex lattices in Bose-Einstein condensates with dipolar interactions beyond the weak-interaction limit, Phys. Rev. A 75, 023623 (2007).

[101] N. R. Cooper, Rapidly rotating atomic gases, Adv. Phys. 57, 539 (2008).

[102] P. Pedri and L. Santos, Two-Dimensional Bright Soliton in Dipolar BoseEinstein Condensates, Phys. Rev. Lett. 95, 200404 (2005).

[103] I. Tikhonenkov, B. A. Malomed, and A. Vardi, Anisotropic Solitons in Dipolar Bose-Einstein Condensates, Phys. Rev. Lett. 100, 090406 (2008). 
[104] R. Nath, P. Pedri, and L. Santos, Soliton-soliton scattering in dipolar BoseEinstein condensates, Phys. Rev. A 76, 013606 (2007).

[105] R. Nath, P. Pedri, and L. Santos, Stability of Dark Solitons in Three Dimensional Dipolar Bose-Einstein Condensates, Phys. Rev. Lett. 101, 210402 (2008).

[106] M. A. Baranov, Theoretical progress in many-body physics with ultracold dipolar gases, Phys. Rep. 464, 71 (2008).

[107] T. Lahaye, C. Menotti, L. Santos, M. Lewenstein, and T. Pfau, The physics of dipolar bosonic quantum gases, Rep. Prog. Phys. 72, 126401 (2009).

[108] M. A. Baranov, M. Dalmonte, G. Pupillo, and P. Zoller, Condensed Matter Theory of Dipolar Quantum Gases, Chem. Rev. 112, 5012 (2012).

[109] S. Ronen, D. C. E. Bortolotti, D. Blume, and J. L. Bohn, Dipolar BoseEinstein condensates with dipole-dependent scattering length, Phys. Rev. A 74, 033611 (2006).

[110] G. E. Astrakharchik, J. Boronat, J. Casulleras, I. L. Kurbakov, and Y. E. Lozovik, Weakly interacting two-dimensional system of dipoles : Limitations of the mean-field theory, Phys. Rev. A 75, 063630 (2007).

[111] D. H. J. O’Dell, S. Giovanazzi, and C. Eberlein, Exact Hydrodynamics of a Trapped Dipolar Bose-Einstein Condensate, Phys. Rev. Lett. 92, 250401 (2004).

[112] C. Eberlein, S. Giovanazzi, and D. H. J. O'Dell, Exact solution of the Thomas-Fermi equation for a trapped Bose-Einstein condensate with dipole-dipole interactions, Phys. Rev. A 71, 033618 (2005).

[113] M. Kreibich, J. Main, and G. Wunner, A variational approach to Bogoliubov excitations and dynamics of dipolar Bose-Einstein condensates, J. Phys. B: At. Mol. Opt. Phys. 46, 045302 (2013).

[114] P. Kapitza, Viscosity of Liquid Helium below the $\lambda$-Point, Nature 141, 74 (1938).

[115] J. F. Allen and A. D. Misener, Flow of Liquid Helium II, Nature 141, 75 (1938).

[116] A. Griffin, Excitations in a Bose-condensed Liquid (Cambridge University Press, 2005).

[117] L. D. Landau, Theory of Superfluidity of Helium II, Phys. Rev. 60, 356 (1941).

[118] L. D. Landau, On the theory of superfluidity of helium II, J. Phys. USSR 11, 91 (1947).

[119] L. Landau, On the theory of superfluidity, Phys. Rev. 75, 884 (1949). 
[120] G. J. Kalman, P. Hartmann, K. I. Golden, A. Filinov, and Z. Donkó, Correlational origin of the roton minimum, EPL 90, 55002 (2010).

[121] R. P. Feynman, Atomic Theory of the Two-Fluid Model of Liquid Helium, Phys. Rev. 94, 262 (1954).

[122] R. P. Feynman and M. Cohen, Energy Spectrum of the Excitations in Liquid Helium, Phys. Rev. 102, 1189 (1956).

[123] M. Cohen and R. P. Feynman, Theory of Inelastic Scattering of Cold Neutrons from Liquid Helium, Phys. Rev. 107, 13 (1957).

[124] F. Zambelli, L. Pitaevskii, D. M. Stamper-Kurn, and S. Stringari, Dynamic structure factor and momentum distribution of a trapped Bose gas, Phys. Rev. A 61, 063608 (2000).

[125] H. Palevsky, K. Otnes, K. E. Larsson, R. Pauli, and R. Stedman, Excitation of Rotons in Helium II by Cold Neutrons, Phys. Rev. 108, 1346 (1957).

[126] H. Palevsky, K. Otnes, and K. E. Larsson, Excitation of Rotons in Helium II by cold neutrons, Phys. Rev. 112, 11 (1958).

[127] D. G. Henshaw and A. D. B. Woods, Modes of Atomic Motions in Liquid Helium by Inelastic Scattering of Neutrons, Phys. Rev. 121, 1266 (1961).

[128] A. D. B. Wood, Neutron Inelastic Scattering from Liquid Helium at Small Momentum Transfers, Phys. Rev. Lett. 14, 355 (1965).

[129] A. D. Martin and P. B. Blakie, Stability and structure of an anisotropically trapped dipolar Bose-Einstein condensate: Angular and linear rotons, Phys. Rev. A 86, 053623 (2012).

[130] F. Ancilotto and F. Toigo, Dipolar Bose gas in a highly anharmonic trap, Phys. Rev. A 89, 023617 (2014).

[131] R. N. Bisset and P. B. Blakie, Fingerprinting Rotons in a Dipolar Condensate: Super-Poissonian Peak in the Atom-Number Fluctuations, Phys. Rev. Lett. 110, 265302 (2013).

[132] J. P. Corson, R. M. Wilson, and J. L. Bohn, Stability spectroscopy of rotons in a dipolar Bose gas, Phys. Rev. A 87, 051605(R) (2013).

[133] J. P. Corson, R. M. Wilson, and J. L. Bohn, Geometric stability spectra of dipolar Bose gases in tunable optical lattices, Phys. Rev. A 88, 013614 (2013).

[134] M. D. Feit, J. A. Fleck Jr., and A. Steiger, Solution of the Schrödinger Equation by a Spectral Method, J. Comput. Phys. 47, 412 (1982).

[135] http://www.netlib.org/lapack/.

[136] T. D. Lee and C. N. Yang, Many-Body Problem in Quantum Mechanics and Quantum Statistical Mechanics, Phys. Rev. 105, 1119 (1957). 
[137] T. D. Lee, K. Huang, and C. N. Yang, Eigenvalues and Eigenfunctions of a Bose System of Hard Spheres and Its Low-Temperature Properties, Phys. Rev. 106, 1135 (1957).

[138] N. Navon, S. Nascimbène, F. Chevy, and C. Salomon, The Equation of State of a Low-Temperature Fermi Gas with Tunable Interactions, Science 328, 729 (2010).

[139] N. Navon, S. Piatecki, K. Günter, B. Rem, T. C. Nguyen, F. Chevy, W. Krauth, and C. Salomon, Dynamics and Thermodynamics of the LowTemperature Strongly Interacting Bose Gas, Phys. Rev. Lett. 107, 135301 (2011).

[140] T. T. Wu, Ground State of a Bose System of Hard Spheres, Phys. Rev. 115, 1390 (1959).

[141] N. M. Hugenholtz and D. Pines, Ground-State Energy and Excitation Spectrum of a System of Interacting Bosons, Phys. Rev. 116, 489 (1959).

[142] K. Sawada, Ground-State Energy of Bose-Einstein Gas with Repulsive Interaction, Phys. Rev. 116, 1344 (1959).

[143] D. S. Petrov, Quantum Mechanical Stabilization of a Collapsing Bose-Bose Mixture, Phys. Rev. Lett. 115, 155302 (2015).

[144] A. R. P. Lima and A. Pelster, Quantum fluctuations in dipolar Bose gases, Phys. Rev. A 84, 041604(R) (2011).

[145] A. R. P. Lima and A. Pelster, Beyond mean-field low-lying excitations of dipolar Bose gases, Phys. Rev. A 86, 063609 (2012).

[146] E. Timmermans, P. Tommasini, and K. Huang, Variational Thomas-Fermi theory of a nonuniform Bose condensate at zero temperature, Phys. Rev. A 55, 3645 (1997).

[147] E. Wigner, On the Quantum Correction For Thermodynamic Equilibrium, Phys. Rev. 40, 749 (1932).

[148] R. N. Bisset and P. B. Blakie, Crystallization of a dilute atomic dipolar condensate, Phys. Rev. A 92, 061603(R) (2015).

[149] K.-T. Xi and H. Saito, Droplet formation in a Bose-Einstein condensate with strong dipole-dipole interaction, Phys. Rev. A 93, 011604(R) (2016).

[150] H. Saito, Path-Integral Monte Carlo Study on a Droplet of a Dipolar BoseEinstein Condensate Stabilized by Quantum Fluctuation, J. Phys. Soc. Jpn. 85, 053001 (2016).

[151] L. Khaykovich, F. Schreck, G. Ferrari, T. Bourdel, J. Cubizolles, L. D. Carr, Y. Castin, and C. Salomon, Formation of a Matter-Wave Bright Soliton, Science 296, 1290 (2002). 
[152] K. E. Strecker, G. B. Partridge, A. G. Truscott, and R. G. Hulet, Formation and propagation of matter-wave soliton trains, Nature(London) 417, 150 (2002).

[153] M. Lu, Quantum Bose and Fermi Gases of Dysprosium: Production and Initial Study, PhD thesis, Stanford University, 2014.

[154] L. Chomaz, S. Baier, D. Petter, M. J. Mark, F. Wächtler, L. Santos, and F. Ferlaino, Quantum-fluctuation-driven crossover from a dilute Bose-Einstein condensate to a macro-droplet in a dipolar quantum fluid, arXiv:1607.06613 (2016).

[155] V. S. Filho, F. Kh. Abdullaev, A. Gammal, and L. Tomio, Autosolitons in trapped Bose-Einstein condensates with two- and three-body inelastic processes, Phys. Rev. A 63, 053603 (2001).

[156] Y. Kagan, B. V. Svistunov, and G. V. Shlyapnikov, Effect of Bose condensation on inelastic processes in gases, Pis'ma Zh. Eksp. Teor. Fiz. 42, 169 (1985).

[157] http://www.fftw.org/. 


\section{Curriculum Vitae}

Address $\quad$ : Kniestr. 22, 30167 Hannover, Germany

Email : falk.waechtler@itp.uni-hannover.de

\section{Personal profile}

Date of Birth : 28th of August 1985

Place of Birth : Dessau, Germany

Gender : Male

Nationality : German

Language : German, English

\section{Education}

$2012-2016$ : PhD, Leibniz Universität Hannover, Germany

$2012-2015$ : Member of Research Training Group 1729: Fundamentals and applications of ultracold matter

$2006-2012$ : Diploma in physics, Universität Potsdam, Germany

2005 : High school graduation (Abitur) at the Gymnasium

"Walter Gropius"-Europaschule, Germany 


\section{Publications}

- L. Chomaz, S. Baier, D. Petter, M. J. Mark, F. Wächtler, L. Santos, F. Ferlaino, Quantum-fluctuation-driven crossover from a dilute Bose-Einstein condensate to a macro-droplet in a dipolar quantum fluid, arXiv:1607.06613, (2016).

- F. Wächtler, L. Santos, Ground-state properties and elementary excitations of quantum droplets in dipolar Bose-Einstein condensates, Phys. Rev. A 94, 043618 (2016).

- F. Wächtler, L. Santos, Quantum filaments in dipolar Bose-Einstein condensates, Phys. Rev. A 93, 061603(R) (2016).

- F. Waechtler, A. R. P. Lima, A. Pelster, Low-Lying Excitation Modes of Trapped Dipolar Fermi Gases: From Collisionless to Hydrodynamic Regime, arXiv:1311.5100, (2013). 


\section{Acknowledgements}

First of all I would like to thank my advisor Prof. Luis Santos for giving me the opportunity to work with him and learn in his group. The completion of this thesis would not have been possible without his guidance and the many constructive advices. My gratitude for all the help and support that I have received from you.

Many thanks also to Daniel. I am very grateful for your constructive criticism of my thesis and the many tips and tricks in order to pretty up the figures.

I also want to thank my collaborators Francesca Ferlaino, Lauriane Chomaz, Simon Baier, Daniel Petter and Manfred Mark. Thanks for the very nice collaboration and fruitful discussions.

Many thanks also to all the people in our and Hendrik's group. Thanks to Lorenzo, Luis and Akos, for sharing the office. I will never forget the many funny moments and interesting discussions that I had with all of you.

I gratefully acknowledge the support from the Institute for Theoretical Physics, the DFG Research Training Group 1729: Fundamentals and applications of ultracold matter headed by Prof. Luis Santos and the people of the RTG for the interesting discussions.

Finally, I want to thank my family for their never ending support and their patience during the last years. I am truly grateful to Robin that you made me curious to learn more about physics and my father who showed me all the interesting literature. 


\section{Selbstständigkeitserklärung}

Hiermit versichere ich, die vorliegende Doktorarbeit selbstständig und unter ausschließlicher Verwendung der angegebenen Hilfsmittel angefertigt zu haben.

Hannover, Oktober 2016

Falk Wächtler 